\title{
WestVirginiaUniversity
}

THE RESEARCH REPOSITORY @ WVU

Graduate Theses, Dissertations, and Problem Reports

2010

\section{Three essays on gay and lesbian rights legislation}

David R. Christafore

West Virginia University

Follow this and additional works at: https://researchrepository.wvu.edu/etd

\section{Recommended Citation}

Christafore, David R., "Three essays on gay and lesbian rights legislation" (2010). Graduate Theses,

Dissertations, and Problem Reports. 4570.

https://researchrepository.wvu.edu/etd/4570

This Dissertation is protected by copyright and/or related rights. It has been brought to you by the The Research Repository @ WVU with permission from the rights-holder(s). You are free to use this Dissertation in any way that is permitted by the copyright and related rights legislation that applies to your use. For other uses you must obtain permission from the rights-holder(s) directly, unless additional rights are indicated by a Creative Commons license in the record and/ or on the work itself. This Dissertation has been accepted for inclusion in WVU Graduate Theses, Dissertations, and Problem Reports collection by an authorized administrator of The Research Repository @ WVU.

For more information, please contact researchrepository@mail.wvu.edu. 


\title{
Three Essays on Gay and Lesbian Rights Legislation
}

\author{
David R. Christafore \\ Dissertation submitted to the \\ College of Business and Economics \\ at West Virginia University in partial \\ fulfillment of the requirements for the degree of
}

Doctor of Philosophy

in

Economics
Santiago Pinto, Ph.D., Chair
Brian Cushing, Ph.D.
Stratford Douglas, Ph.D.
Tami Calvez, Ph.D.
Ming-Jeng Hwang, Ph.D.
Department of Economics
Morgantown, West Virginia
2010

Keywords: Labor, Migration, Spatial Econometrics, Legislation, Sexual Orientation, Hedonic Prices

Copyright 2010 David R. Christafore 


\section{Abstract \\ THREE ESSAYS ON GAY AND LESBIAN RIGHTS LEGISLATION}

\section{David R. Christafore}

This dissertation consists of three essays that provide empirical evidence that informs the ongoing debate over the passage of gay and lesbian rights legislation. In Chapter 2 the difference-in-difference-in-difference methodology is used to examine the effect that the passage of a private employment anti-discrimination law has on the relative earnings and employment of gays and lesbians. The results of this analysis indicate that the passage of this law does not have an impact on either relative earnings or employment for lesbians, but it does have an impact on these labor market outcomes for gays. In particular, the passage of this anti-discrimination law increases the relative earnings for gays by $7.5 \%$ and reduces their relative probability of being employed by $2.4 \%$. In Chapter 3 a gravity model of stateto-state gay and lesbian migration flows over the period 1995-2000 is estimated in order to analyze the revealed preferences of gays and lesbians for private and public employment anti-discrimination, hate crime, and domestic partnership laws. The results of this examination indicate that private employment anti-discrimination laws reduce in-migration, while public employment anti-discrimination and hate crime laws increase in-migration. Therefore, it appears that gays and lesbians prefer to live in places with public employment anti-discrimination and hate crime laws, and not in places with private employment antidiscrimination laws. In Chapter 4 a spatial hedonic autoregressive model is estimated for the Columbus, OH MSA to examine the impact that the percentage of gay and lesbian households in conservative and liberal neighborhoods has on the house prices in those neighborhoods. The results of the analysis indicate that a $.1 \%$ point increase in the percentage of gay and lesbian households increases house prices in liberal neighborhoods by $.81 \%$ and reduces house prices by $.24 \%$ in conservative neighborhoods. Thus, evidence is provided that is consistent with gay and lesbian households being an amenity to liberal neighborhoods, but a disamenity, likely due to prejudice, to conservative neighborhoods. 


\section{Dedication}

I dedicate this dissertation to my wonderful family. Without their love and support I would have never made it through five years of graduate school and completed this dissertation. I also dedicate it to my friends whom have kept me sane throughout all of these years of school by providing me with some much needed opportunities to step back from my work and relax. 


\section{Acknowledgements}

I would like to acknowledge those individuals that contributed to this dissertation. First and foremost, I would like to thank my dissertation advisor Dr. Santiago Pinto. The theoretical and mathematical knowledge that I gained by taking his graduate classes was of immense importance in the development of my research agenda. Also, his comments and encouragement throughout the entire process of writing this dissertation have made it much stronger than it would have ever been without his help.

I would also like to thank Dr. Brian Cushing, Dr. Stratford Douglas, Dr. Hyungai Oh, and Dr. Tami Calvez for serving on my dissertation committee. Their suggestions and criticisms since the initial proposal of this dissertation have allowed it to develop into a much more thorough analysis than what it was originally. Additionally, I would like to extend a special thank you to Dr. Ming-Jeng Hwang for whom my dissertation defense would not be possible without his willingness to serve as a substitute committee member for me.

Last but not least, I must also thank all of those that participated in the April 2009 meeting of the Southern Regional Science Association in San Antonio, TX and in the November 2009 meeting of the Southern Economic Association in San Antonio, TX. The ideas for the second and third chapters of this dissertation were originally presented at these conferences and the discussions that I had at them greatly aided in the development of these ideas. 


\section{Contents}

\begin{tabular}{ll}
\hline Abstract & ii
\end{tabular}

Acknowledgements iv

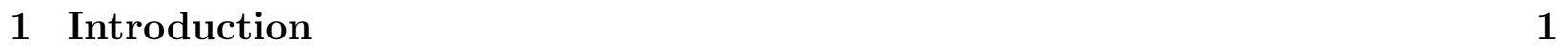

2 An Analysis of the Impact of State Anti-discrimination Laws on the Rel$\begin{array}{ll}\text { ative Earnings and Employment of Gays and Lesbians } & \mathbf{6}\end{array}$

$1 \quad$ Introduction . . . . . . . . . . . . . . . . . . . . . 6

2 Literature Review . . . . . . . . . . . . . . . . . . . . . . . . . . . 8

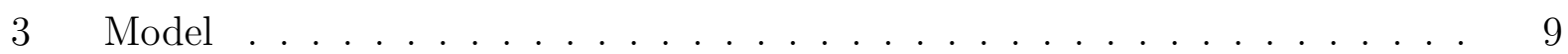

$4 \quad$ Methodology . . . . . . . . . . . . . . . . . . . . . 14

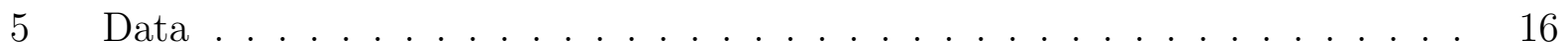

6 Results . . . . . . . . . . . . . . . . . . . . . . . . . 19

7 $\quad$ Conclusion . . . . . . . . . . . . . . . . . . . . . . . . . . . . . . 23

3 A Spatial Migration Model to Investigate the Preferences of Gays and $\begin{array}{ll}\text { Lesbians for State Legislation } & 28\end{array}$

$1 \quad$ Introduction . . . . . . . . . . . . . . . . . . . . . . . . . . . 28

2 Literature Review . . . . . . . . . . . . . . . . . . . . . . . . . . . . . . . . . . . . . . . . . . . .

3 Methodology . . . . . . . . . . . . . . . . . . . . . 33

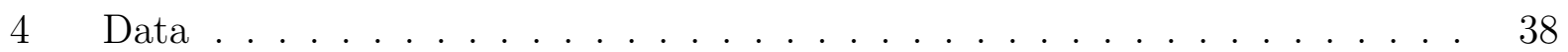

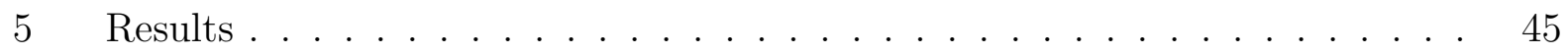

$5.1 \quad$ Descriptive Statistics . . . . . . . . . . . . . . . . . . . . . . . 45

$5.2 \quad$ Main Model Specification . . . . . . . . . . . . . . . . . . . 46

5.3 Checks for Robustness . . . . . . . . . . . . . . . . . . . . . . . 48

6 Conclusion . . . . . . . . . . . . . . . . . . . . . . . . . . . . 51

4 An Examination of Prejudice Against Gay and Lesbian Households: A $\begin{array}{ll}\text { Spatial Hedonic Approach } & 63\end{array}$

1 $\quad$ Introduction . . . . . . . . . . . . . . . . . . . . . . . . . 63

2 Literature Review . . . . . . . . . . . . . . . . . . . . . . 65

$3 \quad$ Data and Methodology . . . . . . . . . . . . . . . . . . . 67

4 4results . . . . . . . . . . . . . . . . . . . . . . . 73

5 Conclusion . . . . . . . . . . . . . . . . . . . 76 


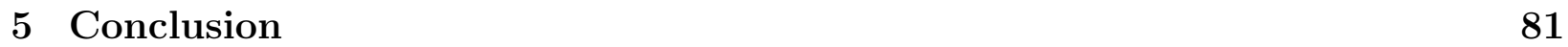




\section{List of Tables}

2.1 Descriptive Statistics for Gays and Lesbians by Year . . . . . . . . . . . . . 24

2.2 Descriptive Statistics for Heterosexuals by Year . . . . . . . . . . . . . . . . 25

2.3 Results for 19-64 Year Old Male and Female Samples . . . . . . . . . . . . . 26

2.4 Results for 19-39 Year Old Male and Female Samples . . . . . . . . . . . . . 26

2.5 Results for 40-64 Year Old Male and Female Samples . . . . . . . . . . . . . 27

2.6 Summary of DDD Estimates for Robustness Checks . . . . . . . . . . . . . . 27

3.1 Source and Magnitude of Top Gay and Lesbian Migrant In-flow and Out-Flow By State . . . . . . . . . . . . . . . . . . . . 53

3.2 Out, In, and Net Gay and Lesbian Migration by State. . . . . . . . . . . . . 54

3.3 Year of Passage of Legislation for States With a Law as of 1996 . . . . . . . 55

3.4 Definition and Source of Variables . . . . . . . . . . . . . . . . . . 56

3.5 Full Sample Bayesian Spatial Autoregressive Tobit Model Estimation Results 57

3.6 (Continued):Full Sample Bayesian Spatial Autoregressive Tobit Model Estimation Results . . . . . . . . . . . . . . . . . . . . 58

$3.7 \quad$ Bayesian Spatial Autoregressive Tobit Model Estimation Results for Separate Gay and Lesbian Samples . . . . . . . . . . . . . . . . . . . . 59

3.8 (Continued): Bayesian Spatial Autoregressive Tobit Model Estimation Results for Separate Gay and Lesbian Samples . . . . . . . . . . . . . . . . . . . . . 60

3.9 Bayesian Spatial Autoregressive Tobit Model Estimation Results Including Local Laws as of 1994 . . . . . . . . . . . . . . . . . . . . . . . . 61

3.10 (Continued): Bayesian Spatial Autoregressive Tobit Model Estimation Results Including Local Laws as of 1994 . . . . . . . . . . . . . . . . . . . . . . . 62

4.1 Definition and Source of Variables . . . . . . . . . . . . . . . . . . . . 78

4.2 Descriptive Statistics $\ldots \ldots \ldots$. . . . . . . . . . . . . . . . . . . 79

4.3 Spatial Autoregressive Hedonic Price Model Results for Cleveland and Columbus MSAs (Dependent Variable is the Natural Log of House Price) . . . . . . 80 


\section{Chapter 1}

\section{Introduction}

The passage of various types of gay and lesbian rights legislation has been a topic of heated debate in recent years. Despite this debate, there has not been much attention paid to issues surrounding the passage of such legislation in the economics literature. This lack of attention is unfortunate since the passage, or non-passage, of gay and lesbian rights legislation can have important impacts on the lives of gays and lesbians. The following three chapters of this dissertation provide empirical evidence that fills this void in the economics literature.

The data set of gays and lesbians that is used in the following three chapters is from the 5\% PUMS of the 1990 and 2000 editions of the U.S. Census. Starting with the 1990 Census, individuals have been able to identify themselves as unmarried partners to the head of the household that they live in. Therefore, gay and lesbian couples can be identified from the Census as those unmarried partners that are of the same sex. Since there is no explicit question asking for a person's sexual orientation, single gays and lesbians cannot be identified and are not a part of this analysis. The reliability of this data set of gay and lesbian unmarried partners has been analyzed in detail by Carpenter (2004) and Black et al. (2000). They both conclude that the Census sample of gay and lesbian couples is representative of gay and lesbian couples in the population and therefore a reliable source of data on these individuals. 
Chapter 2 analyzes the effect that the passage of state laws from 1990-1998 that prohibited private sector labor market discrimination based on a person's sexual orientation had on the relative earnings and employment of gays and lesbians. The motivation for this chapter is that it is not clear a priori if the passage of a sexual orientation anti-discrimination law leads to an improvement or decline in the labor market outcomes of gays and lesbians. The reason for this ambiguity is that the passage of this law increases a firm's expected cost of not hiring gays and lesbians by allowing these individuals to file lawsuits over perceived discrimination in hiring, while at the same time it increases a firm's expected cost of hiring gays and lesbians by allowing these individuals to file lawsuits over perceived discrimination in firing.

The only previous paper to date to analyze the effect of sexual orientation anti-discrimination laws is Klawitter and Flatt (1998), which uses a cross-sectional analysis and focuses mainly on the effect of these laws on gay and lesbian relative earnings. The analysis in Chapter 2 departs from Klawitter and Flatt (1998) in that it adopts the difference-in-difference-indifference methodology that has been commonly used to study the effects that sex (Neumark and Stock, 2001), race (Neumark and Stock, 2001; Collins, 2003), and disability (Beegle and Stock, 2003) anti-discrimination laws have had on protected groups. This methodology provides a more accurate estimate of the effect of the passage of a sexual orientation antidiscrimination law since it allows for pre-law differences in relative earnings and employment between gays and lesbians in states that did and did not pass a law to be controlled for.

The results of the analysis suggest that the passage of a sexual orientation anti-discrimination law does not have an effect on the relative earnings or employment of lesbians, but it does have impact on labor market outcomes for gays. These results also indicate that the impact on gays depends on age. In particular, the passage of a sexual orientation anti-discrimination law increases the relative earnings of gays 19-64 years old by $7.5 \%$ and reduces the relative probability of being employed by $2.4 \%$. Separating the sample into a younger and older group, the passage of this law increases the relative earnings of gays 19-39 years old by 8.6\%, 
but it does not have an effect on the relative earnings of gays 40-64 years old. Also, it reduces the relative probability of being employed for gays 40-64 years old by $4.5 \%$, but it does not have an impact on the relative probability of being employed for gays 19-39 years old.

Chapter 3 examines the preferences of gays and lesbians for sexual orientation private and public employment anti-discrimination, hate crime, and domestic partnership laws. The motivation for this chapter is that the existing evidence on the preferences of gays and lesbians for particular legislation is inconclusive. Survey evidence shows that gays and lesbians face a high rate of hate crimes (Herek, 2009), and econometric studies provide evidence that gays (Elmslie and Tebaldi, 2007; Allegretto and Arthur, 2001) and lesbians (Badgett, 1995) may face labor market discrimination. These studies seem to indicate that gays and lesbians have a strong preference for gay and lesbian rights legislation. However, survey evidence exists that provides evidence that gays and lesbians are not very informed on the existence of state and national gay and lesbian rights legislation (Egan et al., 2008), which suggests that this legislation may not be important to them.

This study is based on the theory, set forth by Tiebout (1956), that individuals reveal their preferences for public sector policies through their location decisions. Following from this theory, the choice of gays and lesbians to migrate in and out of states with and without gay and lesbian rights legislation can be used to analyze their preferences for this legislation. Therefore, a gravity model of state-to-state gay and lesbian migration flows over the period 1995-2000 is estimated. The gravity model is estimated using the spatial autoregressive Tobit model in order to account for spatial dependence in the migration flows of the model as well as the large number of zero migration flows of gays and lesbians that are observed.

The results from the estimation of this model provide evidence that gays and lesbians do have a preference for certain types of gay and lesbian rights legislation. For one, it is found that the inclusion of sexual orientation in hate crime laws and the existence of public employment anti-discrimination legislation increases gay and lesbian in-migration to a state. This finding indicates that gays and lesbians likely benefit from these two pieces 
of legislation. Additionally, the results indicate that the existence of private employment anti-discrimination legislation actually reduces in-migration. This result, therefore, provides evidence that gays and lesbians may be made worse off by this type of legislation, which is a result that is consistent with the findings from Chapter 2, at least for gays, in regard to the impact of private employment anti-discrimination laws.

Chapter 4 uses a revealed preferences approach to analyze the existence of prejudice against gay and lesbian households. The motivation for this chapter is that individuals opposing the passage of gay and lesbian rights anti-discrimination and hate crimes legislation often do so making the claim that gays and lesbians aren't discriminated against in society. Therefore, the argument goes, this legislation just extends special privileges to gays and lesbians. Also, arguments are made against allowing gays and lesbians to marry that make the claim that this legislation would serve to ruin the institution of marriage and negatively impact society. Despite these arguments, empirical evidence suggests that gays (Tebaldi and Elmslie, 2006; Allegretto and Arthur, 2001) and lesbians (Badgett, 1995) face discrimination, are subject to hate crimes (Herek, 2009), and gay and lesbian marriage does not negatively impact society (Langbein and Yost Jr., 2009). Therefore, these arguments might be based more on prejudice that is held against gays and lesbians rather than truth, which one would think is not a valid reason to reject gay and lesbian rights legislation.

The methodology used to analyze the revealed preferences for gay and lesbian households is based on the hedonic price model set forth by Rosen (1974). The hedonic price model suggests that the price of a house represents the sum of expenditures on a number of bundled housing characteristics, including house specific and neighborhood characteristics. Thus, based on this model, an analysis of the implicit price the average household of a neighborhood places on living in a neighborhood with more gay and lesbian households reveals their attitude towards these households. The particular hedonic price model that is estimated in Chapter 4 is estimated using a spatial autoregressive model using data on year 2000 housing transactions in the Columbus, OH MSA. 
The findings of Chapter 4 are consistent with the theory that gay and lesbian households are an amenity to liberal neighborhoods that value diversity, yet a disamentiy to a conservative neighborhood that may hold prejudice against these households. It is found that a .1\% point increase in the percentage of gay and lesbian households in a neighborhood increases house prices in a liberal neighborhood by $.81 \%$. However, in conservative neighborhoods a $.1 \%$ increase in the percentage of gay and lesbian households reduces house prices by $.24 \%$. These results are generally robust to using housing data from the Cleveland, OH MSA, as opposed to the Columbus, OH MSA, for the analysis. 


\title{
Chapter 2
}

\section{An Analysis of the Impact of State}

\author{
Anti-discrimination Laws on the
}

\section{Relative Earnings and Employment of}

\section{Gays and Lesbians}

\section{Introduction}

Gays and lesbians have struggled since the beginning of the 1970's to have legislation passed that prohibits labor market discrimination based on an individual's sexual orientation Rimmerman et al., eds, 2000). Their efforts have had some success at the state level of government as 21 states and the District of Columbia have passed a sexual orientation antidiscrimination law (from here on referred to as a SOA law) (Human Rights Campaign, 2009). This success has not carried over to the federal level of government where a SOA law has yet to be passed:2 Despite the ongoing debate at the federal and state levels of govern-

\footnotetext{
${ }^{1}$ Anti-discrimination laws in this paper refer to those that specifically prohibit employment discrimination.

${ }^{2}$ The Employment Non Discrimination Act (ENDA) is a proposed bill in Congress that would create a federal SOA law. It was first introduced by the 103rd Congress in 1994, and has been introduced by each Congress since then except for the 109th. This proposed bill is currently in the Senate Health, Education,
} 
ment, there is not much evidence that exists on the impact of SOA laws on the earnings and employment of gays and lesbians. This paper provides evidence on the impact of SOA laws by utilizing the difference-in-difference-in-difference (DDD) methodology to examine the effect that the passage of state level SOA laws in the 1990's had on the the relative earnings and employment of gays and lesbians.

A SOA law gives gays and lesbians the ability to file a lawsuit against a firm if they believe that they have been discriminated against in hiring, firing, or pay. Proponents of SOA laws base their support on the belief that gays and lesbians are discriminated against in the labor market (Badgett, 2001). The problem with proponents basing their support on this belief is that even if discrimination exists against gays and lesbians, the passage of a SOA law does not necessarily improve their relative earnings or employment. The reason for this is that the passage of a SOA law may cause the firm to fear a costly lawsuit if it ever decides to fire gays and lesbians after hiring them, resulting in decreased labor demand and worse labor market outcomes for gays and lesbians. In fact, studies on the labor market impacts of disability Acemoglu and Angrist, 2001; Beegle and Stock, 2003) and sex (Neumark and Stock, 2001) anti-discrimination laws provide evidence that the passage of anti-discrimination laws do not always improve labor market outcomes.

To date, the only previous paper to investigate the effects of SOA laws has been Klawitter and Flatt (1998). Their paper uses a cross-sectional analysis to analyze the effect of SOA laws on the relative earnings of gays and lesbians. This paper differs from their paper in that it uses the DDD methodology instead of cross-sectional analysis. The advantage of the DDD methodology is that it allows for differences between gays and lesbians prior to the passage of a SOA law in the states that did and did not pass a SOA law to be controlled for. Controlling for the pre-law differences between these two different groups of gays and lesbians could be important if the passage of SOA laws is correlated with unobservable productivity differences. This paper also contributes to the literature by being the first to examine the

Labor, and Pensions Committee (The Library of Congress, 2009). 
impact that the passage of a SOA law has on gay and lesbian employment.

The DDD methodology can be used to analyze the impact of SOA laws since these laws are passed in eight states between the years 1990 and 1998. The results of the DDD analysis suggest that the passage of a SOA law does not have an effect on the relative earnings or employment of lesbians, but it does have impact on labor market outcomes for gays. In particular, the passage of a SOA law increases the relative earnings of gays 19-39 years old by $8.6 \%$, but it does not have an effect on the relative earnings of gays 40-64 years old. Additionally, the passage of a SOA law reduces the relative probability of being employed for gays 40-64 years old by $4.5 \%$, but it does not have an impact on the relative probability of being employed for gays 19-39 years old.

\section{Literature Review}

A number of previous papers have examined the effect of the passage of the federal racial antidiscrimination law using time-series analysis. Heckman (1990) reviews this literature and concludes that the federal anti-discrimination law improved the economic progress of blacks. The main problem in a time-series analysis of the effect of a federal anti-discrimination law is that it is difficult to determine if the estimated change in the outcome of a particular group is due to the passage of the law or a continuation of a trend for that group that existed prior to the passage of the law. In order to overcome this problem it is necessary to identify individuals of the group that did not experience the passage of the legislation (a "control group"), as well as individuals of the group that did experience the passage of the legislation (a "treatment group"). The identification of these groups is difficult, however, when dealing with federal laws that are mostly universal in their coverage.

Cross-sectional analysis has been applied to looking at the effect of state level laws, which allows for a control group to be identified, but a problem still arises in that differences between the control and treatment groups prior to the passage of the law cannot be identified. 
Cross-sectional analysis is utilized by Klawitter and Flatt (1998) to investigate the effect that SOA laws have on the relative earnings of gays and lesbians. They use data from the $19905 \%$ PUMS of the U.S. Census to regress individual earnings on interaction terms between sexual orientation and the presence of both private and public employment laws, while controlling for individual characteristics and location. The results from their analysis suggest that the presence of a SOA law does not have a significant effect on the relative earnings of gays or lesbians.

Recent studies have estimated regressions with pooled data from different time periods using a difference-in-difference-in-difference methodology. This methodology allows a control group and pre-law differences between the control and treatment group to be identified. Neumark and Stock (2001) employs the DDD methodology to analyze the effect of the passage of sex and race anti-discrimination laws at the state level. They find that race anti-discrimination laws generally increase the relative earnings and employment of blacks and that sex anti-discrimination laws increase relative earnings and decrease relative em-

ployment for women. Collins (2003) applies a similar DDD set up to the study of race anti-discrimination laws and also finds, at least for the 1940's, that the passage of race antidiscrimination laws improved labor market outcomes for blacks. The DDD methodology is also used by Beegle and Stock (2003) in order to study the impact that the passage of disability anti-discrimination laws at the state level have on wages, employment, and labor market participation of the disabled. Their findings are that disability anti-discrimination laws generally lead to lower relative earnings and lower relative labor force participation for the disabled, and they do not have an effect on their relative employment.

\section{Model}

The model for the effect of the passage of a SOA law is based on the model developed by Acemoglu and Angrist (2001) for the effect of disability anti-discrimination laws. Let the 
wage paid to heterosexuals be represented by $w_{a}$ and the wage paid to gays and lesbians $w_{g}$. The labor supply of heterosexuals is then given by $n_{a}\left(w_{a}\right)$ and the labor supply of gays and lesbians $n_{g}\left(w_{g}\right)$. Both $n_{a}$ and $n_{g}$ are assumed to be increasing in $w_{a}$ and $w_{g}$ respectively.

There are assumed to be $M$ firms in the labor market that never exit and a large number of potential firms that can enter the market at cost $\Gamma$. Firms are infinitely lived, risk neutral, and have a discount factor of $\beta<1$. They also have access to a increasing returns to scale production function of the form $F\left(L_{t}, e \cdot G_{t}\right)$, where $L_{t}$ is the number of heterosexuals and $G_{t}$ is the number of gays and lesbians employed by the firm at time $t$. The parameter $e$ in the production function is the relative productivity of gays and lesbians to heterosexuals. The assumption is made that $e \leq 1$, where $e<1$ represents a situation in which the firm has a taste for discrimination based on sexual orientation (Becker, 1971) $\mathrm{H}^{3}$

A SOA law affects the cost to the firm of hiring gays and lesbians. This cost results from the fact that the law allows gays and lesbians to bring lawsuits against firms over perceived discrimination in hiring and firing practices. The probability that a gay or lesbian job applicant that is not hired sues the firm is given by $p_{g}$, and the expected cost of this lawsuit is given by $v_{g}$. Also, the probability that a gay or lesbian that is fired from a job sues the firm is represented by $q_{g}$, and the expected cost of this lawsuit is represented by $\zeta_{g}$. Therefore, the expected cost to the firm of not hiring a gay or lesbian is $h_{g}=p_{g} \cdot v_{g}$, and the expected cost to the firm of firing a gay or lesbian is $f_{g}=q_{g} \cdot \zeta_{g}$. In effect, $h_{g}$ is a hiring subsidy, and $f_{g}$ is a hiring cost faced by the firm. The assumption is made in this paper that heterosexuals do not sue falsely claiming to be gays or lesbians. Thus, the passage of a SOA law does not affect the firm's cost of hiring heterosexuals.

Firms in this model face adverse match-specific shocks that cause the productivity of a worker at his or her current job to fall to 0 with probability $s$ each period. The assumption

\footnotetext{
${ }^{3}$ According to Becker (1971), if employers or workers have a taste for discrimination, then wages are lower for the discriminated against group only if the fraction of discriminating employers is sufficiently large. This wage differential is expected to disappear in the long-run in a competitive market or a market characterized by constant returns to scale. Wage differentials are expected to persist in the long-run if consumers are those with a taste for discrimination.
} 
is made that $(1-\beta) \cdot f_{g}<w_{g}$, so firms that do receive adverse match-specific shocks always lay off the fraction $s$ of their employees. The number of gay and lesbian job applicants is represented by $G_{f}$, and the number of heterosexual job applicant is represented by $L_{f}$, which are both assumed to be given. Also, it is assumed that there is an excess supply of labor in the labor market.

Under these conditions, the maximization problem of a firm at time $t=0$ is

$$
\begin{aligned}
\max _{G_{t}, L_{t}} \Pi= & \sum_{t=0}^{\infty} \beta^{t} \cdot\left(F\left(L_{t}, e \cdot G_{t}\right)-w_{a, t} \cdot L_{t}-w_{g, t} \cdot G_{t}-f_{g} \cdot s \cdot G_{t-1}\right. \\
& \left.-h_{g} \cdot\left\{G_{f}-\left[G_{t}-(1-s) \cdot G_{t-1}\right]\right\}\right) .
\end{aligned}
$$

Following from Acemoglu and Angrist, firms immediately adjust to steady-state employment levels in which $w_{a, t}=w_{a}, w_{d, t}=w_{d}, L_{t}=L$, and $G_{t}=G$ in every period. The equilibrium employment and wage levels are then given by

$$
\begin{aligned}
& \frac{\partial F(L, e \cdot G)}{\partial L}=w_{a} \\
& \frac{\partial F(L, e \cdot G)}{\partial G}=w_{g}+\beta \cdot s \cdot f_{g}-[1-\beta \cdot(1-s)] \cdot h_{g},
\end{aligned}
$$

which equate the marginal products of heterosexual and gay and lesbian workers to their marginal costs.

Market clearing is imposed for heterosexual individuals so

$$
n_{a}^{-1}(m \cdot L)=w_{a}
$$

where $n_{a}^{-1}$ is the inverse supply function, and $m$ is the equilibrium number of firms. The equilibrium number of firms is determined by

$$
\Pi \leq \Gamma, m \geq M
$$


where either the maximized value of profits is equal to entry costs, or there is no entry. In the case of no entry, the number of firms, $m$, is equal to the minimum, $M$.

SOA laws also prohibit discrimination in pay. This equal pay provision also needs to be considered in determining the wages and employment of gays and lesbians. In particular, the wage that gays and lesbians earn is given by

$$
w_{g}=\max \left[n_{g}^{-1}(m \cdot G), \eta \cdot w_{a}\right]
$$

where $\eta$ is the parameter that represents the enforcement of the equal pay provision of the SOA law. If $n_{g}^{-1}(m \cdot G) \geq \eta \cdot w_{a}$, then the equal pay provision is not binding, and equilibrium employment for gays and lesbians is on their labor supply curve. On the other hand, if $n_{g}^{-1}(m \cdot G)<\eta \cdot w_{a}$, then employment for gays and lesbians is off of their labor supply curve resulting in unemployment.

Clearly from the model a number of different scenarios could result for gays and lesbians as the result of the passage of a SOA law. First assume the equal pay provision is not binding. If the effect of the hiring subsidy is greater than the effect of the hiring cost arising from the SOA law, or $\beta \cdot s \cdot f_{g}<[1-\beta \cdot(1-s)] \cdot h_{g}$, then gay and lesbian wages and employment are expected to increase as a result of an increase in labor demand. On the other hand, if the impact of the hiring subsidy is less than the impact of the hiring cost resulting from the SOA law, or $\beta \cdot s \cdot f_{g}>[1-\beta \cdot(1-s)] \cdot h_{g}$, then gay and lesbian wages and employment are expected to decrease as a result of a decrease in labor demand. A case could also exist in which the effect of the hiring subsidy is equal to the effect of the hiring cost. In this case, gay and lesbian wages and employment are expected to be unaffected by the passage of a SOA law.

Now assume that $n_{g}^{-1}(m \cdot G)<\eta \cdot w_{a}$, so that the equal pay provision is binding. A binding equal pay provision by itself acts as a binding minimum wage law as it increases gay and lesbian wages and reduces employment off of the labor supply curve. If a change in 
labor demand for gays and lesbians occurs due to a SOA law and the equal pay provision is binding after this change, then the overall effect of the SOA law on gay and lesbian employment outcomes is not as clear as when the equal pay provision is not binding. In the case that there is an increase in labor demand for gays and lesbians, wages and employment both increase. If wages are still less than heterosexual wages, meaning that the equal pay provision is binding, then wages increase further while employment decreases. Therefore, the overall effect of the SOA law in this case on gays and lesbians is an increase in wages and an ambiguous effect on employment. On the other hand, a decrease in labor demand decreases wages and employment, while the binding equal pay provision further decreases employment but also increases wages. Thus, the overall effect of the SOA law in this situation is a fall in employment and an ambiguous effect on wages.

The theoretical model indicates that the overall effect of the passage of a SOA law on the wages and employment of gays and lesbians depends on whether the impact of the hiring subsidy or the hiring cost is larger and on the impact of the equal pay provision. Data is needed on the prevalence of sexual orientation employment discrimination complaints in order to predict the effect of the passage of a SOA law. Rubenstein (2001) calculates population adjusted discrimination complaint rates for race, gender, and sexual orientation discrimination complaints in states that have a SOA law. He finds that population adjusted complaint rates for sexual orientation discrimination are in the same general range of population adjusted complaint rates for race and gender discrimination. This finding shows that it is likely that firms indeed have a reason to fear lawsuits by gays and lesbians when an SOA law makes these lawsuits possible. Unfortunately, data is not available on the age and sex of those filing the complaints and on if the complaints are over hiring or firing. Thus, it is not possible to predict from the model the effect of the passage of a SOA law. 


\section{Methodology}

The difference-in-difference-in-difference methodology is utilized to investigate the impact of SOA laws on the earnings and employment of gays and lesbians. The object of the DDD methodology in policy analysis is to use a natural experiment to get a clean measure of the effect of the policy in question. A natural experiment took place over the 1990's in that some states passed a SOA law ("treatment states") while other states did not ("control states"). The DDD methodology allows for a comparison of gay and lesbian and heterosexual outcomes (the first difference) in states that did and did not pass a SOA law (the second difference) over time (the third difference). In order to perform the DDD analysis, data is pooled on gays and lesbians and heterosexuals from the 1990 and 2000 Census years.

The DDD equation is given as follows:

$$
\begin{aligned}
& Y_{\text {ist }}=\alpha+X_{i s t} \beta_{1}+\text { SOR }_{\text {ist }} \beta_{2}+\text { State }_{s} \beta_{3}+\text { Year }_{t} \beta_{4}+ \\
& \quad\left(\text { SOR }_{\text {ist }} * \text { State }_{s}\right) \beta_{5}+\left(\text { Year }_{t} * \text { SOR }_{\text {ist }}\right) \beta_{6}+\left(\text { State }_{s} * \text { Year }_{t}\right) \beta_{7}+ \\
& \quad\left(\text { State }_{s} * \text { SOR }_{\text {ist }} * \text { Year }_{t}\right) \beta_{8}+\epsilon_{\text {ist }},
\end{aligned}
$$

where $s$ indexes the two groups of states (those that did and did not pass a SOA law from 1990-1998), $t$ the two time periods (1990 and 2000), and $i$ each individual. In this equation, $Y$ represents either earnings or employment; $S O R$ is a dummy variable that takes the value of 1 if an individual is identified as being gay or lesbian and a 0 otherwise; State is a dummy variable that takes the value of 1 for states that passed a SOA law from 19901998 and 0 otherwise; and Year is a dummy variable that takes the value of 1 for the year 2000 and a value of 0 for the year 1990. Also, $X$ represents a matrix of standard demographic characteristics of individual $i$ for race, age and its square, education 4.4 ability to speak English, the presence of a work limiting disability, the presence of children in the

\footnotetext{
${ }^{4}$ The education variables that are included are dummies for if the individual didn't complete high school, graduated high school, completed some college, earned an undergraduate degree, or earned a post undergraduate degree. Did not complete high school is the omitted dummy from the regression.
} 
household, and residence in a metro area.

Following Collins (2003), in equation (2.6) $\beta_{2}$ represents time and state in-variant differences between gays and lesbians and heterosexuals; $\beta_{3}$ reflects time and sexual orientation in-variant differences between the two groups of states; $\beta_{4}$ captures state group and sexual orientation in-variant differences over time; $\beta_{5}$ represents time in-variant differences between gays and lesbians and heterosexuals in the states that passed an SOA law relative to those that did not; $\beta_{6}$ reflects state in-variant changes in gay and lesbian labor market outcomes relative to heterosexuals; and $\beta_{7}$ captures sexual orientation in-variant changes over time in the group of states that passed an SOA law relative to the group of states that did not. The coefficient of interest in this paper is $\beta_{8}$. This coefficient reflects the change in the labor market outcome for gays and lesbians relative to that of heterosexuals in the states that did and did not pass a law. Therefore, $\beta_{8}$ is the DDD estimate.

Equation (2.6) is estimated for both earnings and employment. The earnings specification is estimated using OLS. The dependent variable in this specification is the log of individual wage and salary earnings for the year prior the the Census $5^{5}$ The analysis is limited to only those individuals that were private sector employees, that had positive earnings, and that were full time employees. Full time employees are defined here as those that usually worked 30 or more hours a week and that worked 27 or more weeks in the previous year. Usual hours worked per week and weeks worked last year are entered as control variables in addition to the ones already mentioned in $X$. The employment specification is estimated using a probit model. In this specification, the dependent variable takes a value of 1 if the individual was employed in the week prior to the Census and a value of 0 otherwise. Only those individuals that are identified as being in the labor force in the week prior to the Census are included in the employment specification.

\footnotetext{
${ }^{5}$ Following from Beegle and Stock (2003), Neumark and Stock (2001), and Collins (2003), the earnings regression is estimated conditional on the individual being employed. The controls to use from the Census in a Heckman selection model are not clear.
} 


\section{Data}

The data necessary for this DDD analysis is from the 1990 and 2000 5\% PUMS of the U.S. Census.6 The Census does not ask individuals directly about their sexual orientation, but beginning with the 1990 Census it has been possible to identify gay and lesbian individuals that are a part of same-sex cohabitating relationships. Individuals in this paper are identified as being gay or lesbian if they are part of a household with two adults of the same sex in which the adults identified themselves as being in an unmarried partnership ${ }^{7}$ neither adult has had his or her age, sex, or relationship status imputed by the Census, both individuals are at least 18 years old, and both live in the United States. Defining gays and lesbians in this way and limiting the sample to just those individuals that are 19-64 years old, due to the focus on labor market outcomes in this paper, allows for the identification of a total of 30,060 gays and 30,480 lesbians from the 1990 and 2000 5\% PUMS.

One issue that arises with using Census data on same-sex cohabitating couples is whether or not these couples are indeed gay and lesbian. Black et al. (2000) and Carpenter (2004) both give an in depth analysis of this issue with both providing evidence that the same-sex cohabitating couples identified on the Census are likely to be gay and lesbian. Black et al. (2000) finds that the residence of $60 \%$ of partnered gays identified on the 1990 Census are concentrated in just 20 cities. This finding wouldn't be expected if those individuals identified as partnered gays are actually a part of the general non-gay population. They also find that there is a strong correlation between the concentration of partnered gays in cities and the concentration of AIDS deaths among young men in those cities. Carpenter (2004) identifies same-sex cohabitating couples from the Center for Disease Control's Behavioral Risk Factor Surveillance System (BRFSS). He shows that these couples are likely to actually be gay and lesbian using information on their sexual behavior and family planning. This

\footnotetext{
${ }^{6}$ This data is publicly available from www.ipums.org.

${ }^{7}$ Gay and lesbian households are identified from the "relationship to household head" heading on the Census. The categories under this heading are spouse, child, inlaw, unmarried partner, and other nonrelative.
} 
finding provides evidence that couples that identify themselves as being same-sex couples on surveys are indeed gay or lesbian.

Another issue that arises is the possibility that some same-sex cohabitating couples fail to report themselves as such due to a distrust of government run surveys. This possibility could make the results of this analysis difficult to interpret if those gay and lesbian couples that identify themselves as same-sex couples on the Census are different in someway from those that do not. Black et al. (2000) shows using data from the General Social Survey (GSS) and the National Health and Social Life Survey (NHSS), which are both non-government run surveys, that in fact the 1990 Census only identifies $35 \%$ of the total number of partnered gay couples and $29 \%$ of the total number of partnered lesbian couples. They discuss, however, that empirical findings for characteristics such as veteran status, education, and earnings for individuals identified as being in same-sex cohabitating couples on the Census are very similar to those for the same-sex cohabitating couples identified in the GSS and NHSS. Additionally, Carpenter (2004) estimates gay and lesbian wage regressions using same-sex couples from the BRFSS and finds that the results for these wage regressions do not differ from the results of similar wage regressions using the same-sex couples that are identified on the Census.

A final issue with using the Census data is that there is no way of knowing if the firm is aware of the sexual orientation of the individuals that are identified as being gay or lesbian. This problem is due to the fact that sexual orientation is different from race or sex in that it must be revealed in someway by the individual to the firm. Therefore, the sexual orientation variable used in the regressions is measured with error and unfortunately there is no way to correct for this measurement error. The hope in this paper is that this measurement error is limited by the fact that only gays and lesbians in cohabitating relationships are used in the analysis: 8

\footnotetext{
${ }^{8}$ This assertion is based on the assumption that gays and lesbians willing to enter into cohabitating relationships are those that are the most open about their sexual orientation, although there is no concrete evidence to back up this assumption.
} 
For the DDD setup in equation (2.6) it is necessary to identify individuals that are heterosexual to use as a comparison group to gays and lesbians. The two different sets of heterosexual individuals that can be identified from the Census are those individuals that are married and those that are in cohabitating relationships with a member of the opposite sex. The group of individuals that are used in this paper as the heterosexual comparison group are those that are in cohabitating relationships with a member of the opposite sex. They are chosen as the comparison group so that differences in labor market outcomes that arise between gays and lesbians and their heterosexual counterparts resulting from the presence of a marriage premium is not present in this analysis (Allegretto and Arthur, 2001). The individuals in heterosexual cohabitating relationships are identified using the same criteria that is used for the same-sex cohabitating couples. This process allows for the identification of a total of 309,383 heterosexual males and 310,123 heterosexual females from the 1990 and $20005 \%$ PUMS.

The laws of interest in this paper are state laws that prohibit labor market sexual orientation discrimination in the private sector. Every state that has passed a private employment SOA law has also passed a public employment SOA law. Also, some states have passed just a public employment SOA law. This paper limits the analysis to just private employment SOA laws because these are the laws that are most likely to have an impact on the labor market outcomes of gays and lesbians, as they cover the most individuals. In addition to states passing SOA laws, a number of cities have also passed SOA laws. Local level laws are not considered in this paper because these laws differ greatly in their level of enforcement at the local level.

The data on the year of passage for SOA laws comes from the NGLTF Policy Institute (1998) and the Human Rights Campaign (2009). Only those states that passed a SOA law between the years 1990 and 1998 are counted as being states that passed a SOA law for the DDD analysis. From 1990 to 1998, the eight states that passed a SOA law were Connecticut (1991), Hawaii (1991), California (1992), New Jersey (1992), Vermont (1992), Minnesota 
(1993), Rhode Island (1995), and New Hampshire (1998). Nevada passed a SOA law in 1999, but because this is the same year as the reference year for the reported earnings on the 2000 Census Nevada is counted as not passing a SOA law. Also, it is clear from equation (2.6) that observations from states that passed a SOA law prior to 1990 cannot be included in the analysis. Therefore, observations from Minnesota (1989), Wisconsin (1982), and the District of Columbia (1977) are excluded from the DDD analysis.9

Table 2.1 reports variable descriptive statistics for gays and lesbians, and table 2.2 reports these statistics for heterosexual males and females. The descriptive statistics in each table are grouped by Census year. Descriptive statistics for earnings are calculated for just those individuals that had positive earnings, worked 27 or more weeks, 30 or more hours per week, and earned a positive income in the previous year. Just those individuals that are part of the labor force are included in the calculation of the employment statistics.

\section{Results}

The results for the estimation of the earnings and employment specifications of equation (2.6) are reported in table 2.3 for males and females 19-64 years old. The individual productivity controls used in each regression are statistically significant with the expected signs. The coefficient on the triple interaction term $S O R *$ State $*$ Year is the DDD estimate for the impact of the passage of a SOA law. The DDD estimates for the male sample reveal that the passage of a SOA law leads to a $7.5 \%$ increase in the relative earnings of gays. This increase in earnings is accompanied by a $2.5 \%$ reduction in the relative probability of being employed. As for lesbians, the evidence suggests that a SOA law does not have an impact on relative earnings or employment.

To further investigate the impact of a SOA law, each specification of equation (2.6) is

\footnotetext{
${ }^{9}$ In all, 21 states and the District of Columbia have passed a SOA law to date. The states that have passed a SOA law in addition to the ones already mentioned are Maryland (2001), New Mexico (2003), New York (2003), Maine (2005), Illinois (2006), Washington (2006), Colorado (2007), Iowa (2007), Oregon (2008), and Delaware (2009).
} 
re-estimated for a 19-39 year old sample and a 40-64 year old sample of males and females. The results for these re-estimations are reported in tables 2.4 and 2.5 respectively. The results suggest that age does indeed have an influence on the impact of a SOA law on gays. In particular, gays 19-39 years old experience a 8.6\% increase in relative earnings without an accompanying change in the relative probability of being employed. On the other hand, gays 40-64 years old experience a 4.5\% decline in the relative probability of being employed without a change in relative earnings. Turning to the female 19-39 and 40-64 year old results, the results again show that a SOA law does not have an effect on the relative earnings or probability of being employed for lesbians.

The theoretical model presented in section 3 predicts that a simultaneous increase in relative earnings and decrease in relative employment, as is observed for gays in the full male sample regressions, is the result of a binding equal pay provision of a SOA law. The statistically significant negative coefficient on the $S O R$ variable in table 2.3 for males suggests that the equal pay provision of a SOA law is indeed likely to be binding for gays. The evidence also suggests that the rise in relative earnings for gays goes entirely to younger gays and the reduction in relative employment falls entirely on older gays. Perhaps one reason for this age difference is that older gays are easier to layoff because firms can claim the layoff is due to declining productivity with age.

Provided in table 2.6 are the DDD estimates obtained from re-estimating the earnings and employment specifications of equation (2.6) for a number of robustness checks of the main results. One issue that arises in this analysis is that gays and lesbians could migrate to states that have passed a SOA law if that law is leading to improved relative earnings and employment outcomes. This migration may offset any increases in relative earnings or employment that result from the passage of the SOA law. In order to deal with this issue, each of the main regressions is re-estimated excluding those individuals from the year 2000 that had migrated from a different state within the last five years. The results from this re-estimation are consistent in statistical significance and similar in magnitudes to the main 
results, suggesting that migration is not likely an issue in this analysis.

Another issue is regional differences may be influencing the main DDD estimates. States located in some regions of the country appear to be more inclined than others to have passed a SOA law by 1998. Therefore, the main DDD estimates may be picking up regional changes in the relative earnings and employment of gays and lesbians instead of the effect of a SOA law. This possibility is dealt with by re-estimating equation (2.6) adding regional dummies for the northeast, south, midwest, and west. Also, interactions are included in the re-estimation between the regional dummies and the sexual orientation dummy, time dummy, and the sexual orientation and time interaction. In this re-estimation, statistical significance is unchanged with the exception that the DDD estimate for the full male sample employment specification is insignificant. As a result of including the regional controls, the increase in relative earnings for gays 19-39 years old jumps to $16.6 \%$ and the decrease in the relative probability of being employed for gays 40-64 years old increases to $6.2 \%$.

An additional issue is that the inclusion of New Hampshire as a state that passed a SOA law could be biasing the DDD estimates. New Hampshire passed their SOA law in 1998. A law passed this late in the time period under consideration may not have given the law enough time to have an impact on the relative earnings and employment of gays and lesbians. This factor is taken into consideration by re-estimating the main regressions including New Hampshire in the group of states that did not pass a law. The DDD estimates from this re-estimation are again consistent in statistical significance and similar in magnitudes to the main results.

Also, occupational controls have not been included in the earnings specification of the main regressions because they may be endogenous. Leaving out occupational controls presents a problem, however, in that occupations could be correlated with the passage of a SOA law, resulting in an omitted variables problem. This problem could arise if sexual orientation discrimination exists in certain occupations, and the passage of a SOA law allows gays and lesbians to enter into these occupations. Therefore, the earnings specification of the 
main regressions is re-estimated including occupational controls ${ }^{10}$ There is not any change in statistical significance from the main results for earnings as a result of this re-estimation.

A last issue is that there is the possibility that the passage of a SOA law is endogenous. There are two reasons why this might be the case. One reason is that SOA laws could be less costly to pass in states where gays and lesbians are performing relatively well in the labor market and not facing discrimination. Another reason could be that SOA laws are passed in response to gays and lesbians experiencing labor market discrimination and performing relatively worse in the labor market. An examination of the passage of SOA laws over the 1990's shows that they tended to be enacted in traditionally more liberal states. Thus, if endogeneity does exist, it is likely to be the result of the first reason mentioned.

Evidence on the issue of endogeneity is provided by applying the technique used in Beegle and Stock (2003) and Neumark and Stock (2001). In this technique, individual earnings and employment in 1990 are regressed on the same control variables from $X$ in equation (2.6), individual state dummies, and a set of interaction terms between the individual state dummies and sexual orientation dummy. The resulting coefficients on the state dummy and sexual orientation dummy interaction terms are considered to represent year 1990 regression adjusted state earnings and employment gaps for gays and lesbians. A binary variable for if a state passed a SOA law by 1998 or not is then regressed on these regression adjusted employment and earnings gaps using a probit model. This procedure is performed for both male and female samples of the earnings and employment specifications. The results show that 1990 gay earnings gaps, 1990 gay employment gaps, 1990 lesbian earnings gaps, and 1990 lesbian employment gaps all do not have a statistically significant impact on the passage of SOA law by 1998. These results provide evidence that the passage of SOA laws is unlikely to be endogenous.

\footnotetext{
${ }^{10}$ The occupational variables that are included are dummies for if the individual worked in a managerial and professional specialty occupation; technical, sales, and administrative support occupation; service occupation; precision production, craft, and repair occupation; operator, fabricator, and laborer occupation; and farming, forestry, and fishing occupation. The managerial and professional specialty occupation is the omitted dummy from the regression.
} 


\section{Conclusion}

This chapter improves upon previous studies of sexual orientation private employment antidiscrimination (SOA) laws by being the first to use the difference-in-difference-in-difference (DDD) methodology to analyze the impact of these laws. The benefit of using this methodology as opposed to cross sectional analysis is that differences in gay and lesbian employment outcomes in states that did and did not have a law that existed before any laws were passed can be controlled for, allowing for a cleaner measure of the impact of the law. Evidence is provided that indicates that the passage of a SOA law has an impact on labor market outcomes for gays and does not have an impact on labor market outcomes for lesbians. The results from the main DDD regressions indicate that the passage of a SOA law causes the wages of gays relative to heterosexual males to increase and the employment of gays relative to heterosexual males to decrease. When the sample of gays is divided into an older and younger sample, the results show that the increase in relative wages is experienced by the younger gays, and the decrease in relative employment is experienced by the older gays. The magnitudes of these effects are economically significant as the increase in relative wages for younger gays is $8.6 \%$, and the decrease in the relative probability of being employed for older gays is $4.5 \%$.

The analysis presented in this chapter, therefore, indicates that the passage of SOA laws generally do not have the intended effect of improving the labor market outcome for gays and lesbians. The theoretical model that is developed shows that this result is possible due to the unintended effects of the SOA law on the incentives of firms to hire gays and lesbians. This result has important implications for gay and lesbian rights groups that are expending resources in order to have SOA laws passed. In particular, it suggests that a more effective use of their resources may be on directly lobbying employers to not discriminate or on a general education of society on sexual orientation discrimination. Use of resources in these ways are unlikely to have the negative unintended consequences that the passage of SOA laws can have. 
Table 2.1: Descriptive Statistics for Gays and Lesbians by Year

\begin{tabular}{lcccc}
\hline & Gay 1990 & Gay 2000 & Lesbian 1990 & Lesbian 2000 \\
\hline Earnings & $37504.87^{\mathrm{a}}$ & 38592.21 & 32878.92 & 38592.21 \\
& $(472.78)^{\mathrm{b}}$ & $(296.09)$ & $(390.48)$ & $(296.09)$ \\
Hours & 43.42 & 44.32 & 42.49 & 43.58 \\
& $(0.13)$ & $(0.07)$ & $(0.14)$ & $(0.07)$ \\
Weeks & 49.66 & 50.05 & 44.32 & 49.76 \\
& $(0.08)$ & $(0.04)$ & $(0.07)$ & $(0.04)$ \\
Employed & 0.9495 & 0.9580 & 0.9555 & 0.9611 \\
& $(0.0031)$ & $(0.0016)$ & $(0.0038)$ & $(0.0015)$ \\
Age & 35.87 & 40.28 & 39.53 & 35.27 \\
& $(0.13)$ & $(0.08)$ & $(0.07)$ & $(0.15)$ \\
Child & 0.0594 & 0.1575 & 0.1215 & 0.2296 \\
& $(0.0031)$ & $(0.0026)$ & $(0.0052)$ & $(0.0029)$ \\
High School degree & 0.1526 & 0.2163 & 0.1538 & 0.2159 \\
& $(0.0048)$ & $(0.0030)$ & $(0.0058)$ & $(0.0028)$ \\
Some college & 0.2619 & 0.2256 & 0.2318 & 0.2344 \\
& $(0.0059)$ & $(0.0030)$ & $(0.0067)$ & $(0.0030)$ \\
College degree & 0.3188 & 0.2837 & 0.3215 & 0.2738 \\
Advanced degree & $(0.0062)$ & $(0.0033)$ & $(0.0074)$ & $(0.0031)$ \\
& 0.1597 & 0.1298 & 0.1863 & 0.1419 \\
Disability & $(0.0049)$ & $(0.0024)$ & $(0.0062)$ & $(0.0024)$ \\
& 0.0854 & 0.1286 & 0.0827 & 0.1125 \\
English & $(0.0036)$ & $(0.0024)$ & $(0.0044)$ & $(0.00220)$ \\
Black & 0.9944 & 0.9856 & 0.9957 & 0.9890 \\
& $(0.0010)$ & $(0.0009)$ & $(0.0010)$ & $(0.0007)$ \\
Metro & 0.0628 & 0.0865 & 0.0828 & 0.1066 \\
& $(0.0035)$ & $(0.0021)$ & $(0.0046)$ & $(0.0022)$ \\
Observations & 0.9101 & 0.8470 & 0.8502 & 0.8098 \\
& $(0.0036)$ & $(0.0025)$ & $(0.0056)$ & $(0.0026)$ \\
\hline \multirow{2}{*}{ Nean } & 6497 & 23563 & 4664 & 25819 \\
\hline
\end{tabular}

${ }^{a}$ Means are reported for all continuous variables and proportions for all binary variables.

${ }^{\mathrm{b}}$ Standard errors are in parentheses. 
Table 2.2: Descriptive Statistics for Heterosexuals by Year

\begin{tabular}{|c|c|c|c|c|}
\hline & Male 1990 & Male 2000 & Female 1990 & Female 2000 \\
\hline Earnings & $\begin{array}{c}31290.20^{\mathrm{a}} \\
(92.18)^{\mathrm{b}}\end{array}$ & $\begin{array}{c}34160.37 \\
(94.84)\end{array}$ & $\begin{array}{c}23459.58 \\
(64.59)\end{array}$ & $\begin{array}{c}26642.65 \\
(72.21)\end{array}$ \\
\hline Hours & $\begin{array}{l}44.19 \\
(0.03)\end{array}$ & $\begin{array}{l}44.73 \\
(0.03)\end{array}$ & $\begin{array}{l}41.20 \\
(0.03)\end{array}$ & $\begin{array}{l}41.49 \\
(0.02)\end{array}$ \\
\hline Weeks & $\begin{array}{l}49.10 \\
(0.02)\end{array}$ & $\begin{array}{l}49.54 \\
(0.02)\end{array}$ & $\begin{array}{l}49.03 \\
(0.02)\end{array}$ & $\begin{array}{l}49.24 \\
(0.02)\end{array}$ \\
\hline Employed & $\begin{array}{c}0.9168 \\
(0.0009)\end{array}$ & $\begin{array}{c}0.9344 \\
(0.0007)\end{array}$ & $\begin{array}{c}0.9209 \\
(0.0010)\end{array}$ & $\begin{array}{c}0.9279 \\
(0.0008)\end{array}$ \\
\hline Age & $\begin{array}{l}34.18 \\
(0.03)\end{array}$ & $\begin{array}{c}35.33 \\
(0.03)\end{array}$ & $\begin{array}{l}32.16 \\
(0.03)\end{array}$ & $\begin{array}{c}33.80 \\
(0.03)\end{array}$ \\
\hline Child & $\begin{array}{c}0.1826 \\
(0.0012)\end{array}$ & $\begin{array}{c}0.2203 \\
(0.0011)\end{array}$ & $\begin{array}{c}0.2845 \\
(0.0014)\end{array}$ & $\begin{array}{c}0.2742 \\
(0.0011)\end{array}$ \\
\hline High School degree & $\begin{array}{c}0.3246 \\
(0.0015)\end{array}$ & $\begin{array}{c}0.3317 \\
(0.0012)\end{array}$ & $\begin{array}{c}0.3237 \\
(0.0015)\end{array}$ & $\begin{array}{c}0.3046 \\
(0.0012)\end{array}$ \\
\hline Some college & $\begin{array}{c}0.2199 \\
(0.0014)\end{array}$ & $\begin{array}{c}0.2351 \\
(0.0011)\end{array}$ & $\begin{array}{c}0.2469 \\
(0.0014)\end{array}$ & $\begin{array}{c}0.2681 \\
(0.0011)\end{array}$ \\
\hline College degree & $\begin{array}{c}0.1715 \\
(0.0012)\end{array}$ & $\begin{array}{c}0.1765 \\
(0.0010)\end{array}$ & $\begin{array}{c}0.1839 \\
(0.0013)\end{array}$ & $\begin{array}{c}0.2012 \\
(0.0010)\end{array}$ \\
\hline Advanced degree & $\begin{array}{c}0.0455 \\
(0.0007)\end{array}$ & $\begin{array}{c}0.0429 \\
(0.0005)\end{array}$ & $\begin{array}{c}0.0364 \\
(0.0006)\end{array}$ & $\begin{array}{c}0.0454 \\
(0.0005)\end{array}$ \\
\hline Disability & $\begin{array}{c}0.0757 \\
(0.0008)\end{array}$ & $\begin{array}{c}0.1316 \\
(0.0009)\end{array}$ & $\begin{array}{c}0.0650 \\
(0.0008)\end{array}$ & $\begin{array}{c}0.1146 \\
(0.0008)\end{array}$ \\
\hline English & $\begin{array}{c}0.9946 \\
(0.0002)\end{array}$ & $\begin{array}{c}0.9919 \\
(0.0002)\end{array}$ & $\begin{array}{c}0.9919 \\
(0.0003)\end{array}$ & $\begin{array}{c}0.9872 \\
(0.0003)\end{array}$ \\
\hline Black & $\begin{array}{c}0.1490 \\
(0.0012)\end{array}$ & $\begin{array}{c}0.1542 \\
(0.0010)\end{array}$ & $\begin{array}{c}0.1309 \\
(0.0011)\end{array}$ & $\begin{array}{c}0.1298 \\
(0.0009)\end{array}$ \\
\hline Metro & $\begin{array}{c}0.7510 \\
(0.0014)\end{array}$ & $\begin{array}{c}0.7706 \\
(0.0010)\end{array}$ & $\begin{array}{c}0.7511 \\
(0.0014)\end{array}$ & $\begin{array}{c}0.7698 \\
(0.0010)\end{array}$ \\
\hline Observations & 118432 & 190951 & 118392 & 191731 \\
\hline
\end{tabular}

${ }^{a}$ Means are reported for all continuous variables and proportions for all binary variables.

b Standard errors are in parentheses. 
Table 2.3: Results for 19-64 Year Old Male and Female Samples

\begin{tabular}{|c|c|c|c|c|c|c|c|c|}
\hline & \multicolumn{2}{|c|}{ Male Earnings } & \multicolumn{2}{|c|}{ Male Employment ${ }^{c}$} & \multicolumn{2}{|c|}{ Female Earnings } & \multicolumn{2}{|c|}{ Female Employment } \\
\hline & Coefficient & t-stat $^{\mathrm{a}}$ & $\mathrm{dF} / \mathrm{dx}$ & z-stat & Coefficient & t-stat & $\mathrm{dF} / \mathrm{dx}$ & z-stat \\
\hline Age & 0.0597 & $65.08^{* * * b}$ & 0.0009 & $2.65^{* * *}$ & 0.0577 & $63.92^{* * *}$ & 0.0025 & $6.97^{* * *}$ \\
\hline Age squared & -0.0006 & $-47.28^{* * *}$ & 0.0000 & -1.54 & -0.0006 & $-48.90^{* * *}$ & 0.0000 & $-3.11 * * *$ \\
\hline Child & 0.0256 & $8.47^{* * *}$ & 0.0125 & $10.60^{* * *}$ & -0.0212 & $-7.39^{* * *}$ & -0.0092 & $-7.83^{* * *}$ \\
\hline High School degree & 0.1778 & $50.51^{* * *}$ & 0.0307 & $26.37^{* * *}$ & 0.1961 & $45.63^{* * *}$ & 0.0423 & $34.39 * * *$ \\
\hline Some college & 0.2593 & $67.36^{* * *}$ & 0.0434 & $35.16^{* * *}$ & 0.3140 & $71.06^{* * *}$ & 0.0563 & $45.05^{* * *}$ \\
\hline College degree & 0.4713 & $110.10^{* * *}$ & 0.0574 & $43.76^{* * *}$ & 0.5524 & $117.99^{* * *}$ & 0.0717 & $54.57^{* * *}$ \\
\hline Advanced degree & 0.7029 & $92.50^{* * *}$ & 0.0550 & $28.72^{* * *}$ & 0.7805 & $110.99^{* * *}$ & 0.0597 & $33.48^{* * *}$ \\
\hline Disability & -0.0613 & $-13.25^{* * *}$ & -0.0041 & $-2.37 * *$ & -0.0714 & $-14.78^{* * *}$ & 0.0054 & $2.89 * * *$ \\
\hline English & 0.3771 & $23.81^{* * *}$ & 0.0167 & $2.88^{* * *}$ & 0.3407 & $21.16^{* * *}$ & 0.0590 & $9.36 * * *$ \\
\hline Black & -0.1409 & $-38.08^{* * *}$ & -0.0509 & $-31.56^{* * *}$ & -0.0414 & $-10.44^{* * *}$ & -0.0412 & $-24.28^{* * *}$ \\
\hline Metro & 0.1279 & $43.95^{* * *}$ & 0.0161 & $13.79^{* * *}$ & 0.1730 & $61.15^{* * *}$ & 0.0124 & $10.37^{* * *}$ \\
\hline Weeks & 0.0317 & $118.73^{* * *}$ & $\mathrm{x}$ & $\mathrm{x}$ & 0.0320 & $121.68^{* * *}$ & $\mathrm{x}$ & $\mathrm{x}$ \\
\hline Hours & 0.0119 & $70.11^{* * *}$ & $\mathrm{x}$ & $\mathrm{x}$ & 0.0162 & $68.22^{* * *}$ & $\mathrm{x}$ & $\mathrm{x}$ \\
\hline SOR & -0.0517 & $-4.64^{* * *}$ & 0.0081 & $1.81^{*}$ & 0.0609 & $4.71^{* * *}$ & 0.0051 & 0.92 \\
\hline Year & 0.0535 & $17.62^{* * *}$ & 0.0149 & $12.26^{* * *}$ & 0.0724 & $24.28^{* * *}$ & 0.0028 & $2.28^{* *}$ \\
\hline State & 0.1414 & $29.49^{* * *}$ & -0.0034 & $-1.84^{*}$ & 0.1655 & $35.33^{* * *}$ & 0.0014 & 0.69 \\
\hline $\mathrm{SOR}^{*}$ Year & 0.0426 & $3.31^{* * *}$ & 0.0055 & 1.04 & 0.0504 & $3.57^{* * *}$ & 0.0145 & $2.52^{* *}$ \\
\hline SOR*State & -0.0261 & -1.37 & 0.0034 & 0.44 & -0.0136 & -0.64 & 0.0098 & 0.85 \\
\hline State*Year & -0.0587 & $-9.35^{* * *}$ & -0.0059 & $-2.39^{* *}$ & -0.0459 & $-7.44^{* * *}$ & -0.0100 & $-3.76^{* * *}$ \\
\hline SOR ${ }^{*}$ State*Year & 0.0750 & $3.21^{* * *}$ & -0.0235 & $-2.19^{* *}$ & 0.0235 & 0.96 & -0.0166 & -1.13 \\
\hline Observations & 251713 & & 299008 & & 218477 & & 267078 & \\
\hline $\mathrm{R}$ squared & 0.32 & & & & 0.40 & & & \\
\hline
\end{tabular}

${ }^{a}$ t-stats and z-stats are calculated using robust standard errors.

b Statistical significance is reported at the $1 \%(* * *), 5 \%(* *)$, and $10 \%(*)$ levels.

${ }^{c} \mathrm{dF} / \mathrm{dx}$ probabilities are reported for the probit estimation of employment specifications. The probabilities are calculated for binary variables by switching these variables from 0 to 1 .

Table 2.4: Results for 19-39 Year Old Male and Female Samples

\begin{tabular}{|c|c|c|c|c|c|c|c|c|}
\hline & \multicolumn{2}{|c|}{ Male Earnings } & \multicolumn{2}{|c|}{ Male Employment ${ }^{c}$} & \multicolumn{2}{|c|}{ Female Earnings } & \multicolumn{2}{|c|}{ Female Employment } \\
\hline & Coefficient & t-stat ${ }^{a}$ & $\mathrm{dF} / \mathrm{dx}$ & z-stat & Coefficient & t-stat & $\mathrm{dF} / \mathrm{dx}$ & z-stat \\
\hline Age & 0.1098 & $36.46^{* * * \mathrm{~b}}$ & 0.0080 & $6.64^{* * *}$ & 0.1120 & $39.80^{* * *}$ & 0.0080 & $6.74^{* * *}$ \\
\hline Age squared & -0.0014 & $-27.74^{* * *}$ & -0.0001 & $-6.17^{* * *}$ & -0.0015 & $-31.19^{* * *}$ & -0.0001 & $-5.27 * * *$ \\
\hline Child & 0.0101 & $2.91^{* * *}$ & 0.0118 & $8.39^{* * *}$ & -0.0429 & $-12.90 * * *$ & -0.0149 & $-10.14^{* * *}$ \\
\hline High School degree & 0.1623 & $40.15^{* * *}$ & 0.0336 & $24.36^{* * *}$ & 0.1797 & $35.65^{* * *}$ & 0.0485 & $32.31^{* * *}$ \\
\hline Some college & 0.2309 & $52.52^{* * *}$ & 0.0489 & $33.36^{* * *}$ & 0.2764 & $53.84^{* * *}$ & 0.0653 & $42.81^{* * *}$ \\
\hline College degree & 0.4408 & $89.41^{* * *}$ & 0.0627 & $39.71^{* * *}$ & 0.5083 & $93.31^{* * *}$ & 0.0830 & $51.03^{* * *}$ \\
\hline Advanced degree & 0.6341 & $65.05^{* * *}$ & 0.0595 & $22.17^{* * *}$ & 0.6966 & $78.66^{* * *}$ & 0.0648 & $27.69 * * *$ \\
\hline Disability & -0.0479 & $-8.65^{* * *}$ & 0.0004 & 0.19 & -0.0579 & $-10.04^{* * *}$ & 0.0109 & $4.58^{* * *}$ \\
\hline English & 0.3411 & $17.62^{* * *}$ & 0.0096 & 1.37 & 0.3286 & $17.41 * * *$ & 0.0587 & $7.54^{* * *}$ \\
\hline Black & -0.1326 & $-30.31^{* * *}$ & -0.0619 & $-31.04^{* * *}$ & -0.0360 & $-8.03^{* * *}$ & -0.0464 & $-22.16^{* * *}$ \\
\hline Metro & 0.1144 & $34.91 * * *$ & 0.0166 & $11.79 * * *$ & 0.1620 & $50.18^{* * *}$ & 0.0144 & $9.80 * * *$ \\
\hline Weeks & 0.0318 & $107.47^{* * *}$ & $\mathrm{x}$ & $\mathrm{x}$ & 0.0321 & $110.86^{* * *}$ & $\mathrm{x}$ & $\mathrm{x}$ \\
\hline Hours & 0.0122 & $62.26^{* * *}$ & $\mathrm{x}$ & $\mathrm{x}$ & 0.0168 & $60.61^{* * *}$ & $\mathrm{x}$ & $\mathrm{x}$ \\
\hline SOR & -0.0536 & $-4.24 * * *$ & 0.0025 & 0.46 & 0.0451 & $3.23^{* * *}$ & 0.0027 & 0.39 \\
\hline Year & 0.0686 & $20.21 * * *$ & 0.0159 & $10.96^{* * *}$ & 0.0757 & $22.78^{* * *}$ & 0.0045 & $3.02^{* * *}$ \\
\hline State & 0.1411 & $26.55^{* * *}$ & -0.0044 & $-2.00 * *$ & 0.1664 & $32.32^{* * *}$ & 0.0035 & 1.53 \\
\hline $\mathrm{SOR}^{*}$ Year & 0.0457 & $2.94^{* * *}$ & 0.0028 & 0.43 & 0.0401 & $2.55^{* *}$ & 0.0196 & $2.78 * * *$ \\
\hline SOR*State & -0.0198 & -0.90 & 0.0007 & 0.07 & -0.0009 & -0.04 & 0.0145 & 0.97 \\
\hline State*Year & -0.0676 & $-9.56^{* * *}$ & -0.0051 & $-1.72^{*}$ & -0.0532 & $-7.69 * * *$ & -0.0128 & $-3.92^{* * *}$ \\
\hline SOR*State*Year & 0.0858 & $3.06^{* * *}$ & -0.0092 & -0.73 & 0.0344 & 1.21 & -0.0304 & -1.46 \\
\hline Observations & 176011 & & 207081 & & 156466 & & 192925 & \\
\hline $\mathrm{R}$ squared & 0.32 & & & & 0.40 & & & \\
\hline
\end{tabular}

${ }^{\mathrm{a}}$ t-stats and z-stats are calculated using robust standard errors.

b Statistical significance is reported at the $1 \%(* * *), 5 \%(* *)$, and $10 \%(*)$ levels.

${ }^{\mathrm{c}} \mathrm{dF} / \mathrm{dx}$ probabilities are reported for the probit estimation of employment specifications. The probabilities are calculated for binary variables by switching these variables from 0 to 1 . 
Table 2.5: Results for 40-64 Year Old Male and Female Samples

\begin{tabular}{|c|c|c|c|c|c|c|c|c|}
\hline & \multicolumn{2}{|c|}{ Male Earnings } & \multicolumn{2}{|c|}{ Male Employment ${ }^{c}$} & \multicolumn{2}{|c|}{ Female Earnings } & \multirow{2}{*}{\multicolumn{2}{|c|}{ Female Employment }} \\
\hline & Coefficient & t-stat ${ }^{\mathrm{a}}$ & $\mathrm{dF} / \mathrm{dx}$ & z-stat & Coefficient & t-stat & & \\
\hline Age & 0.0453 & $6.56^{* * * \mathrm{~b}}$ & -0.0008 & -0.37 & 0.0460 & $6.38^{* * *}$ & 0.0074 & $3.06^{* * *}$ \\
\hline Age squared & -0.0004 & $-5.84^{* * *}$ & 0.0000 & 0.70 & -0.0004 & $-5.87 * * *$ & -0.0001 & $-2.51^{* *}$ \\
\hline Child & 0.0541 & $8.88^{* * *}$ & 0.0151 & $7.16^{* * *}$ & 0.0156 & $2.71^{* * *}$ & 0.0083 & $4.34^{* * *}$ \\
\hline High School degree & 0.2127 & $30.13^{* * *}$ & 0.0246 & $11.34^{* * *}$ & 0.2285 & $27.94^{* * *}$ & 0.0273 & $12.77^{* * *}$ \\
\hline Some college & 0.3245 & $41.47^{* * *}$ & 0.0297 & $12.94^{* * *}$ & 0.3996 & $46.01^{* * *}$ & 0.0333 & $15.27^{* * *}$ \\
\hline College degree & 0.5249 & $61.72^{* * *}$ & 0.0428 & $17.9^{* * *}$ & 0.6218 & $66.66^{* * *}$ & 0.0413 & $18.30^{* * *}$ \\
\hline Advanced degree & 0.7891 & $64.82^{* * *}$ & 0.0461 & $15.80^{* * *}$ & 0.8930 & $76.33^{* * *}$ & 0.0454 & $17.05^{* * *}$ \\
\hline Disability & -0.0846 & $-10.19^{* * *}$ & -0.0134 & $-4.70^{* * *}$ & -0.0940 & $-10.76^{* * *}$ & -0.0058 & $-2.06^{* *}$ \\
\hline English & 0.4719 & $18.19^{* * *}$ & 0.0379 & $3.59^{* * *}$ & 0.3881 & $13.30 * * *$ & 0.0656 & $6.19^{* * *}$ \\
\hline Black & -0.1556 & $-22.57 * * *$ & -0.0284 & $-10.5^{* * *}$ & -0.0535 & $-6.51^{* * *}$ & -0.0298 & $-10.57^{* * *}$ \\
\hline Metro & 0.1573 & $26.25^{* * *}$ & 0.0135 & $6.62^{* * *}$ & 0.1944 & $33.81^{* * *}$ & 0.0055 & $2.79^{* * *}$ \\
\hline Weeks & 0.0310 & $52.45^{* * *}$ & $\mathrm{x}$ & $\mathrm{x}$ & 0.0310 & $50.50^{* * *}$ & $\mathrm{x}$ & $\mathrm{x}$ \\
\hline Hours & 0.0110 & $33.35^{* * *}$ & $\mathrm{x}$ & $\mathrm{x}$ & 0.0145 & $31.96^{* * *}$ & $\mathrm{x}$ & $\mathrm{x}$ \\
\hline SOR & -0.0452 & $-1.98^{* *}$ & 0.0186 & $2.37^{* *}$ & 0.1068 & $3.62^{* * *}$ & 0.0070 & 0.79 \\
\hline Year & 0.0191 & $2.90^{* * *}$ & 0.0144 & $6.44^{* * *}$ & 0.0768 & $11.54^{* * *}$ & -0.0002 & -0.09 \\
\hline State & 0.1402 & $13.24^{* * *}$ & -0.0001 & -0.03 & 0.1588 & $14.66^{* * *}$ & -0.0060 & -1.62 \\
\hline SOR*Year & 0.0406 & 1.64 & 0.0057 & 0.62 & 0.0326 & 1.06 & 0.0075 & 0.80 \\
\hline SOR*State & -0.0463 & -1.25 & 0.0093 & 0.69 & -0.0518 & -1.16 & 0.0038 & 0.23 \\
\hline State*Year & -0.0415 & $-3.13^{* * *}$ & -0.0085 & $-1.89^{*}$ & -0.0328 & $-2.45^{* *}$ & -0.0017 & -0.38 \\
\hline SOR*State*Year & 0.0675 & 1.58 & -0.0446 & $-2.23^{* *}$ & 0.0300 & 0.62 & 0.0013 & 0.07 \\
\hline Observations & 75702 & & 91927 & & 62011 & & 74153 & \\
\hline $\mathrm{R}$ squared & 0.24 & & & & 0.32 & & & \\
\hline
\end{tabular}

${ }^{a}$ t-stats and z-stats are calculated using robust standard errors.

${ }^{\mathrm{b}}$ Statistical significance is reported at the $1 \%(* * *), 5 \%(* *)$, and $10 \%(*)$ levels.

${ }^{c} \mathrm{dF} / \mathrm{dx}$ probabilities are reported for the probit estimation of employment specifications. The probabilities are calculated for binary variables by switching these variables from 0 to 1 .

Table 2.6: Summary of DDD Estimates for Robustness Checks

\begin{tabular}{lccccccc}
\hline & \multicolumn{2}{c}{ Migrants Excluded } & \multicolumn{2}{c}{ W/Regions } & \multicolumn{2}{c}{ NH Switched } & W/Occupations \\
Sample & Earnings & Employment $^{\mathrm{c}}$ & Earnings & Employment & Earnings & Employment & Earnings \\
\hline Male 19-64 & $0.0729^{* * * \mathrm{~b}}$ & $-0.0221^{* * *}$ & $0.1339^{* * *}$ & -0.0224 & $0.0691^{* * *}$ & $-0.0222^{* * *}$ & $0.0780^{* * *}$ \\
& $(3.05)^{\mathrm{a}}$ & $(-2.00)$ & $(4.10)$ & $(-1.41)$ & $(2.95)$ & $(-2.08)$ & $(3.42)$ \\
Male 19-39 & $0.0882^{* * *}$ & -0.0089 & $0.1664^{* * *}$ & -0.0052 & $0.0769^{* * *}$ & -0.0078 & $0.0914^{* * *}$ \\
& $(2.98)$ & $(-0.66)$ & $(4.26)$ & $(-0.29)$ & $(2.73)$ & $(-0.62)$ & $(3.32)$ \\
Male 40-64 & 0.0591 & $-0.0381^{*}$ & 0.0732 & $-0.0615^{*}$ & 0.0688 & $-0.0446^{* *}$ & 0.0590 \\
& $(1.38)$ & $(-1.95)$ & $(1.26)$ & $(-1.95)$ & $(1.61)$ & $(-2.23)$ & $(1.41)$ \\
Female 19-64 & 0.0125 & -0.0153 & 0.0184 & -0.0004 & 0.0222 & -0.0131 & 0.0191 \\
& $(0.50)$ & $(-1.04)$ & $(0.64)$ & $(-0.02)$ & $(0.90)$ & $(-0.90)$ & $(0.80)$ \\
Female 19-39 & 0.0243 & -0.0302 & 0.0320 & -0.0144 & 0.0267 & -0.0226 & 0.0243 \\
& $(0.83)$ & $(-1.42)$ & $(0.94)$ & $(-0.58)$ & $(0.92)$ & $(-1.12)$ & $(0.87)$ \\
Female 40-64 & 0.0212 & 0.0019 & 0.0173 & 0.0138 & 0.0380 & -0.0001 & 0.0428 \\
& $(0.44)$ & $(0.11)$ & $(0.32)$ & $(0.66)$ & $(0.78)$ & $(-0.01)$ & $(0.91)$ \\
\hline
\end{tabular}

${ }^{a}$ t-stats and z-stats are calculated using robust standard errors.

${ }^{\mathrm{b}}$ Statistical significance is reported at the $1 \%(* * *), 5 \%(* *)$, and $10 \%(*)$ levels.

${ }^{\mathrm{c}} \mathrm{dF} / \mathrm{dx}$ probabilities are reported for the probit estimation of employment specifications. The probabilities are calculated for binary variables by switching these variables from 0 to 1 . 


\section{Chapter 3}

\section{A Spatial Migration Model to Investigate the Preferences of Gays and Lesbians for State Legislation}

\section{Introduction}

Gay and lesbian rights groups lobby for legislation to be passed at all levels of government that would extend certain rights and protections to gay and lesbian individuals. The pieces of legislation that are most commonly sought after are those that allow gays and lesbians to enter into legally recognized relationships, increase the punishment for hate based crimes, and that outlaw sexual orientation discrimination in employment and other facets of life. The passage of such legislation is often met with fierce resistance by conservative groups and politicians alike. Due to this fierce resistance, legislation granting these rights and protections to gays and lesbians has not gained widespread passage at the local, state, or federal levels of government. In fact, the only gay and lesbian rights law to be enacted at the federal level is a sexual orientation hate crime law that was signed into law on October 
28, 2009, after years of debate $t^{1}$ Debate over the passage of other gay and lesbian rights legislation at all levels of government continues today $\left.\right|^{2}$ Despite this ongoing debate, little is known on the actual preferences of gays and lesbians for the passage of particular legislation.

Knowledge on the preferences of gays and lesbians for particular laws is important to both gay and lesbian rights groups and politicians. Gay and lesbian rights groups need to know on which types of legislation, if any, to use their limited resources on in attempting to get passed. Politicians should have an idea of if the costs involved with debating and enacting particular legislation result in actual benefits for gays and lesbians. Furthermore, state and local politicians can use information on the laws gays and lesbians benefit from in order to try to attract these individuals to their regions. Attracting gays and lesbians may be desirable because research has shown that locations with a larger creative class, which gays and lesbians are often assumed to be a part of, experience higher economic growth (Florida, 2002).

One method that can be used to determine the preferences of gays and lesbians for legislation is to infer those preferences from existing survey and empirical data on the existence of discrimination against these individuals. This method is problematic, however, as the evidence from existing research on the presence of discrimination against gays and lesbians is mixed. Evidence pointing towards the existence of discrimination is provided by Herek (2009), which shows using survey data that gays and lesbians at least perceive themselves as being targets of hate crimes. Also, Tebaldi and Elmslie (2006) and Allegretto and Arthur (2001) provide evidence that gays face labor market discrimination and results from Badgett (1995) indicate that both gays and lesbians are subject to labor market discrimination. On the other hand, Klawitter and Flatt (1998) finds that the presence of private employment anti-discrimination laws at a location do not have an effect on employment outcomes of gays

\footnotetext{
${ }^{1}$ The Local Law Enforcement Hate Crimes Prevention Act of 2009 was passed as an Amendment to the National Defense Authorization Act for the Fiscal Year 2010 (The Library of Congress, 2010).

${ }^{2}$ The Employment Non Discrimination Act (ENDA) is a proposed bill in Congress that would create a federal law making it a crime to discriminate in private employment based on an individual's sexual orientation. This bill was first introduced by the 103rd Congress in 1994 and has been introduced by each Congress since then, except for the 109th (The Library of Congress, 2009).
} 
and lesbians, suggesting that discrimination against gays and lesbians may not exist.

Inference concerning the preferences of gays and lesbians for legislation can also be made using survey results concerning the knowledge of gays and lesbians on the existence of certain gay and lesbian rights and protection laws. If gays and lesbians truly have a preference for the passage of legislation, then the expectation is that they would be knowledgeable on at least the gay and lesbian rights laws of the country and state that they reside in. In a nationally representative survey conducted by Egan et al. (2008), of the 768 gays, lesbians, and bisexuals questioned, only $59 \%$ of the respondents knew that there was not a federal law that prohibited discrimination based on sexual orientation at the time of the survey. Additionally, only $38 \%$ of poll respondents answered all four questions on the existence of particular state and federal laws that were asked on the survey correctly. These low poll results provide evidence that gays and lesbians don't invest much time in learning about legislation. This finding seems to suggest that certain types of rights and protection legislation may not be important to gays and lesbians.

Another approach that can be taken to analyze the preferences of gays and lesbians is to study their revealed preferences for particular legislation through their migration decisions. This approach is based on the model set forth by Tiebout (1956), which suggests that individuals "vote with their feet" for particular public policies. This revealed preferences method for analyzing gay and lesbian preferences has yet to be used in the literature and is the method that is used in this paper. The benefit of this revealed preferences approach is that examining the actual choices of gays and lesbians is likely to provide more insight into their preferences rather than trying to infer these preferences.

The particular migration model that is used is a gravity model of gay and lesbian state-tostate migration flows from 1995-2000. The specific gay and lesbian rights and protections laws that are the focus of this paper are those that varied between states as of 1994. These laws are hate crime laws, private employment anti-discrimination laws, public employment antidiscrimination laws, and domestic partnership laws. The gravity model of gay and lesbian 
state-to-state migration flows is estimated using the spatial autoregressive Tobit model. The spatial autoregressive model is used because there is the likelihood of spatial dependence occurring in the migration flows of gays and lesbians due to sharing of information between gays and lesbians at proximate locations. The Tobit version of this model is employed because it appears from the large number of zero state-to-state gay and lesbian migration flows in the data that gays and lesbians receive negative utility from making certain state-to-state moves.

The results from the estimation of this model provide evidence that gays and lesbians do have a preference for certain types of gay and lesbian rights legislation. In particular, evidence is provided from the main model specification that sexual orientation hate crime laws and public employment anti-discrimination laws increase in-migration, while private employment anti-discrimination laws reduce in-migration. Additionally, it is found that the existence of domestic partnership laws do not have an impact on in-migration or outmigration. These results are generally robust to re-estimations of the main model using different cutoff years for the existence of legislation, separate gay and lesbian samples, and using variable definitions for legislation that include local legislation in addition to state legislation.

\section{Literature Review}

The only study to date that has taken an econometric approach to analyzing the location of gays and lesbians is Black et al. (2002), which specifically focused on the location of gay households. Their paper argues that gay households demand less housing space than heterosexual households because gay households are less likely to have children. Therefore, gay households have more income to spend on adult related amenities than heterosexual households. This difference in economic constraints between gay and heterosexual households leads to gay households concentrating in cities that have higher levels of adult related 
amenities. Regressions are used that regress a citys gay household concentration on the amenities and the citizen attitudes towards homosexuality in a city. The results from these regressions provide evidence that amenities have a strong positive effect on gay concentration in a city, but citizen attitudes do not have an effect on this concentration. The same general results are found for lesbian households as well, although the amenity effect is not as strong since lesbian households are more likely to have children than gay households.

There are issues with Black et al. (2002) that make it a less than ideal study of factors important for gay and lesbian location. For one, it does not take into consideration the effect of the presence of gay and lesbian legislation at a particular location. According to Tiebout (1956), individuals sort themselves into locations based on their preferences for the public policies offered at that location. Therefore, the presence of different types of legislation at a location possibly influences gay and lesbian location decisions differently than the general populations. This legislation is likely correlated with amenities and the public attitude toward homosexuality, confounding the results of the study. Another issue is that a regression of only the concentration of gays and lesbians at a particular time on a set of variables is likely to suffer from endogeneity. It is not possible to tell if gays and lesbians are located in the city because of amenities, attitudes, or legislation, or if these attributes of the city have resulted from gays and lesbians choosing to locate in the city for some other reason.

The approach often used to analyze the revealed preferences of individuals for specific public policies is to study their migration decisions. Examples of public policies that have been analyzed in the migration literature are the effect of government taxation and expenditures on elderly migration (Conway and Houtenville, 2001; Conway and Rork, 2006; Clark and Hunter, 1992; Conway and Houtenville, 2003), the effect of welfare benefits on the migration of female headed households (Blank, 1988) and the migration of the poor Cebula and Koch, 1989), and the effect of various economic freedom indicators on overall migration (Ashby, 2007). Migration models in the regional science literature typically use the gravity 
model of migration as a starting point. This model is derived from the equation for gravitational force from the physical sciences and explains origin to destination migration as a function of the population at the two locations and the distance between them Greenwood, 1975; Borjas, 1989). The gravity model is normally modified to include controls for other locational attributes likely to affect migration decisions. The other attributes that have commonly been found to impact migration and that have been included in gravity models are

those concerning economic opportunity (Fields, 1979; Greenwood et al., 1986; Greenwood and Hunt, 1989), natural amenities such as climate (Graves, 1979; Cushing, 1987), and cost of living (Fournier et al., 1988).

Cushing and Poot (2004) points out that one issue with the current migration literature is that spatial econometric techniques have been under utilized. This under utilization is a clear problem as LeSage and Pace (2008) provides evidence that migration models that control for spatial dependence are superior to those that do not. Ashby (2007) is one migration study that does incorporate spatial econometrics. He develops a methodology that can be used to apply spatial econometrics to a state-to-state migration flows model and a similar methodology to his is used in this paper. This model is used in Ashby (2007) to analyze migration responses to measures of state economic freedom and evidence is provided that individuals "vote with their feet" by migrating to states with relatively higher government expenditures and transfers, smaller tax burdens, and lower minimum wages and union densities.

\section{Methodology}

The typical gravity model of state-to-state migration flows takes the following reduced form:

$$
\begin{aligned}
& m_{i j}=\alpha_{0}+\alpha_{i} p_{i}+\alpha_{j} p_{j}+ \\
& \alpha_{d} d_{i j}+\beta_{i} X_{i}(.)+\beta_{j} X_{j}(.)+u_{i j}
\end{aligned}
$$


where $m_{i j}$ is the migrant flow from origin state $i$ to destination state $j, p_{i}$ is the population of the origin, $p_{j}$ is the population of the destination, $d_{i j}$ is the distance between the origin and destination, and $X_{i}($.$) and X_{j}($.$) include a set of other characteristics of i$ and $j$ that potentially influences migration flows between the two locations. The distance between the origin and destination is included on the right hand to control for the psychic and monetary costs of migration, as these costs are likely to increase with the distance of a move. Origin and destination populations are included because origins with larger populations experience more out migration and those migrants are likely attracted to other places with large populations. Other origin and destination characteristics that are typically included in gravity models include controls for economic opportunity, amenities, cost of living, and public policies at the origin and destination. Migration flows and population often enter into the equation in $\log$ form, while the other independent variables enter in level or log form.

Gravity models of origin-destination flows have historically been estimated using OLS. The use of OLS results in biased and inefficient parameter estimates though in the presence of spatially dependent variables. Migration flows are likely to exhibit spatial dependence as individuals in states located near each other are culturally and economically similar and are therefore likely to migrate to similar locations. Recent research has shown that spatial dependence is not fully accounted for by only controlling for distance in the OLS model (Lee and Pace, 2005; LeSage and Pace, 2009: Tiefelsdorf, 2003). Results from these studies suggest that spatial econometric techniques that include a spatial lag of the dependent variable are more appropriate than OLS in estimating gravity models of origin-destination flows.

When using spatial econometric models a weight matrix must be constructed that represents the spatial dependence that exists in the dependent variable. This spatial weight matrix is usually an $n \times n$ matrix that represents spatial connectivity using a distance, contiguity, or closest neighbor criteria. The selection of the weight matrix to use in the model is often done ad hoc and a priori by the researcher. One way in which the $n \times n$ weight matrix is often constructed is as a standardized first order rook contiguity matrix. This type of 
weight matrix is constructed by entering a value of one into the matrix if two locations share a border with each other and a zero into the matrix if they do not. The weight matrix is then standardized by dividing the entries across each row by the number of non-zero values in the row.

In an origin-destination flows model the typical $n \times n$ weight matrix must be transformed into an $n^{2} \times n^{2}$ matrix. This transformation is necessary because for each pair of locations $i$ and $j$ in the model there are two observations that are included in the model. One observation is included for the flow of migrants from $i$ to $j$ and another observation for the flow of migrants from $j$ to $i$. The way this transformation is performed depends on the type of spatial dependence in the origin-destination flows that is assumed. One possible weight matrix, $W_{o}$, can be constructed as the spatial dependence of an origin to destination flow on the average of flows from the neighbors of the origin to the destination. Another possible weight matrix, $W_{d}$, is one that captures the spatial dependence of an origin to destination flow on the average of flows from the origin to the neighbors of the destination. A third possibility is a weight matrix $W_{w}$ that captures the influence of the average of flows from the neighbors of the origin to the neighbors of the destination on the origin to destination flow.

Following from Ashby (2007), the model that is estimated in this paper uses the $W_{o}$ spatial weight matrix. The reason for assuming the spatial relationship takes this form is that it is likely gays and lesbians that are located in neighboring locations have contact with each other and share information about possible migration destinations. Therefore, the migration flows from neighboring origins to the same destination are the most likely flows to exhibit dependence. A Kronecker product of the form $W \otimes I_{n}$, where $W$ is the $n \times n$ standardized first order rook contiguity matrix and $I_{n}$ is an $n$ dimension identity matrix, is used to create the $n^{2} \times n^{2} W_{o}$ matrix $!^{3}$

In a typical state-to-state migration model an observation is included for a migration flow from each of the 49 origin states (the continental 48 states and the District of Columbia,

\footnotetext{
${ }^{3}$ The creation of the $W_{o}$ matrix in this manner assumes that the dependent variable vector is formed so that it is sorted first by each origin location and then by each destination location.
} 
which from here on is considered to be a state) to each of the 48 destination states, resulting in 2352 observations. An adjustment must be made though when using a spatial model of origin-destination flows since the weight matrix used in this model must be a square matrix. The adjustment that is used in this paper follows from Ashby (2007) in that an observation is included for the migration flow from each of the 49 origin states to themselves. Included in this observation is the number of individuals that did not migrate out of the state over the migration period. This number is calculated by subtracting gay and lesbian migrants into the state over the period 1995-2000 from the 2000 state gay and lesbian population. In order to separate the effect of these 49 stayer observations from the 2352 flow observations that are of interest, the constant is excluded from the model and in its place two dummy variables are included that represent if a migration flow observation represents those that are staying in the origin state or those that are migrating from the origin to destination.

An issue that is encountered when estimating this model is that $56 \%$ of the values on the dependent variable of gay and lesbian state-to-state migration flows are zero. The large number of zero flows suggests that the use of the typical spatial autoregressive model in analyzing gay and lesbian migration results in biased estimates. The approach that is employed to deal with this issue is to use the Tobit model to estimate the regression equation, as is suggested by LeSage and Pace (2009). The motivation for the use of the Tobit Model in this situation is that individuals in certain origin states can be thought of as receiving negative utility from moving to certain destination states. Therefore, non-zero gay and lesbian stateto-state migration flows are observed with some probability and the positive flows take on a continuous distribution. 
The spatial autoregressive Tobit model for the gravity model takes the form:

$$
\begin{aligned}
& m_{i j}=\left\{\begin{array}{c}
0 \text { if } m_{i j}^{*} \leq 0 \\
m_{i j}^{*} \text { if } m_{i j}^{*}>0
\end{array}\right\} \\
& m_{i j}^{*}=\rho W_{o} m_{i j}^{*}+\alpha_{s} \text { stayer }+\alpha_{m} \text { mover }+\alpha_{i} p_{i}+\alpha_{j} p_{j}+ \\
& \alpha_{d} d_{i j}+\beta_{o} X_{i}(.)+\beta_{d} X_{j}(.)+u_{i j}, \text { where } u_{i j} \text { is } N\left(0, \sigma^{2} I_{n}\right) .
\end{aligned}
$$

A Bayesian estimation approach is used to estimate the spatial autoregressive Tobit model because the maximum likelihood estimation of this model is difficult (LeSage and Pace, 2009). Diffuse priors are used for the Bayesian estimation because no prior information is known for the parameters of the model. The interpretation of the results from Bayesian estimation differ from their interpretation in the classical approach in that the concept of a confidence interval does not exist in the Bayesian approach as it does in the classical approach. The significance level in Bayesian estimation is based on a posterior density interval. This interval is interpreted as an interval the researcher is $95 \%$ (for a $5 \%$ significance level) certain to contain the true value of the coefficient being estimated (Kennedy, 2008).

The use of a spatial model allows direct, indirect, and total effects for each of the independent variables to be calculated. The direct effect represents the average effect that a change in the independent variable of an observation has on it's own dependent variable. This coefficient includes the initial impact of the change in an independent variable on it's dependent variable as well as feedback in the system. This feedback occurs when the change in the dependent variable causes changes in the other dependent variables of the system through the spatial weight matrix that in turn feedback onto the initial dependent variable. The indirect effect represents the average spatial spill over effect that a change in an independent variable has on all other dependent variables, excluding it's own. The total effect is the sum of the direct and indirect effects. The results for the direct effects are the main focus of this paper due to the interest in gay and lesbian preferences. 


\section{Data}

The focus of this paper is on the migration flows of the gay and lesbian population. The data on gay and lesbian state-to-state migration flows is from the 5\% PUMS of the 2000 U.S. Census.4 The Census does not ask individuals directly about their sexual orientation, but beginning with the 1990 Census it has been possible to identify gay and lesbian individuals that are a part of same-sex cohabitating relationships. Individuals are identified as being gay or lesbian if they are part of a household with two adults of the same sex in which the adults identified themselves as being in an unmarried partnership $5^{5}$ neither adult has had his or her age, sex, or relationship status imputed by the Census, both individuals in the household reside in the continental United States, and both are between the ages 25-65. Defining gays and lesbians in this way and using Census sampling weights allows for the identification of 930,917 gays and lesbians residing in the United States in the year 2000.

One issue that arises with using Census data on same-sex cohabitating couples is whether or not these couples are indeed gay and lesbian. Black et al. (2000) and Carpenter (2004) both give an in depth analysis of this issue with both providing evidence that the same-sex cohabitating couples identified on the Census are likely to be gay and lesbian. Black et al. (2000) finds that the residence of $60 \%$ of partnered gays identified on the 1990 Census are concentrated in just 20 cities. This finding wouldn't be expected if those individuals identified as partnered gays were actually a part of the general non-gay population. They also find that there is a strong correlation between the concentration of partnered gays in cities and the concentration of AIDS deaths among young men in those cities. Carpenter (2004) identifies same-sex cohabitating couples from the Center for Disease Control's Behavioral Risk Factor Surveillance System (BRFSS) and shows that these couples are likely to be gay and lesbian using information on the sexual behavior and family planning of these couples. This finding

\footnotetext{
${ }^{4}$ This data is publicly available from www.ipums.org.

${ }^{5}$ Gay and lesbian households are identified from the "relationship to household head" heading on the Census. The categories under this heading are spouse, child, inlaw, unmarried partner, and other nonrelative.
} 
provides evidence that couples that identify themselves as being same-sex couples on surveys are indeed gay or lesbian.

Another issue that arises is the possibility that some same-sex cohabitating couples fail to report themselves as such due to a distrust of government run surveys. This possibility could make the results of this analysis difficult to interpret if those gay and lesbian couples that identify themselves as same-sex couples on the Census are different in someway from those that do not. Black et al. (2000) shows using data from the General Social Survey (GSS) and the National Health and Social Life Survey (NHSS), which are both non-government run surveys, that in fact the 1990 Census only identifies $35 \%$ of the total number of partnered gay couples and $29 \%$ of the total number of partnered lesbian couples. They discuss, however, that empirical findings for characteristics such as veteran status, education, and earnings for individuals identified as being in same-sex cohabitating couples on the Census are very similar to those for the same-sex cohabitating couples identified in the GSS and NHSS. Also, Carpenter (2004) estimates gay and lesbian wage regressions using same-sex couples from the BRFSS and finds that the results for these wage regressions do not differ from the results of similar wage regressions using the same-sex couples that are identified on the Census.

Migration moves in this paper are calculated from the PUMS as those gay and lesbian individuals that lived in a different state than their current residence 5 years prior to the 2000 Census. Individual state-to-state migration moves are identified in this fashion and summed to get the aggregate state-to-state migration flows between the years 1995-2000. Table 3.1 shows the top out-migrant and in-migrant flow of each state, while table 3.2 provides data on the total gay and lesbian out-migration, in-migration, and net-migration for each state. Also provided in tables 3.1 and 3.2 is an estimate of the gay and lesbian population in each state at the beginning of the migration period. The method that is used for this estimation is to add the number of non-migrants to the number of out-migrants for each state.

The explanatory variables of interest in this paper are those that represent state gay and lesbian rights and protection legislation. As of 1994, the four areas of rights and protections 
for gays and lesbians that states differed in their laws regarding were public employment antidiscrimination laws, private employment anti-discrimination laws, domestic partnership laws, and hate crime laws. States that had passed private employment anti-discrimination laws had in most cases also passed some combination of other private market anti-discrimination laws regarding housing markets, credit markets, union practices, and education. These other anti-discrimination laws are highly correlated with the private employment law and including all of these laws in the analysis presents an issue with multi-collinearity. Thus, out of these laws only the presence of a private employment anti-discrimination law is included as an explanatory variable.

The data on the presence of public employment anti-discrimination, private employment anti-discrimination, hate crime, and domestic partnership laws in states and the years in which the laws were passed comes from the National Gay and Lesbian Task Force (1998) and Rimmerman et al., eds (2000). Provided in table 3.3 is a list of the states that had and had not passed each of these types of laws by 1994 and the years in which the laws were passed. These laws enter into the regression as separate origin and destination dummy variables that take the value of one if the state had passed the particular law by 1994 and that take a value of zero if they had not.

The year 1994 is used as the cutoff year for two primary reasons. First off, the migration data from the $5 \%$ PUMS only allows the migration of an individual to be identified from that person living in a different location than they did five years previously. This identification process means that it is not possible to know the year in which the person migrated to their 2000 residence. If for example 1996 is used as the cutoff for legislation and an individual migrated in 1995, then bias is introduced into the coefficients. Also, 1994 is used as the cutoff to reduce the possibility of endogenity between the migration flows and the passage of the laws. Endogeneity could exist as an increase in gay and lesbian migration into a state increases the gay and lesbian population, which in turn gives them more political power to pass legislation. However, since gays and lesbians are a relatively small portion of the 
population endogeneity in this form is likely not much of an issue.

Domestic partnership laws entitle the partners of gays and lesbians to some or all of the same employer benefits often offered to the spouses and children of married individuals. The three states that had passed a law allowing for gays and lesbians to enter into domestic partnerships as of 1994 were Vermont, Massachusetts, and the District of Columbia. An issue that arises in interpreting the results for this variable is that some employers voluntarily offer domestic partnership benefits in the absence of a state law. Therefore, it may appear in the data that an individual is moving to a location without this law, when in reality the employer at the location they are moving to is providing domestic partner benefits. Nevertheless, the results for this variable still give an indication of the effect that legal recognition at the state level of gay and lesbian relationships has on the migration of gays and lesbians. The expectation then is that the presence of a law allowing for domestic partnerships in a state increases the migration flow of gays and lesbians into the state and reduces the flow out of the state. This result should especially be true for those gays and lesbians that are in partnered relationships and these are the gays and lesbians that are the focus of this paper.

As of 1994, 16 states had a hate crime law that included crimes that are based on a person's sexual orientation as a hate crime ${ }^{6}$ Sexual orientation hate crime laws legislate that in crimes in which the victim is specifically targeted because of his or her sexual orientation the perpetrator of the crime receives a harsher penalty than he or she normally would. The reason for this harsher penalty is that it is believed that hate crimes cause especially harsh psychological damages to the victims of the crimes and to those individuals that are a part of the entire gay and lesbian community. Since the prevalence of hate crimes against gays and lesbians may be correlated, either positively or negatively, with the passage of a hate crime law, data should be included into the analysis on the prevalence of hate crimes. Unfortunately, accurate sexual orientation hate crime data is not available at the state level

\footnotetext{
${ }^{6}$ Arizona, Maryland, and Utah included sexual orientation in their hate crimes data collection law as of 1994, but not in the state's penalty laws. Also, Texas's hate crime law could be interpreted as including sexual orientation but was basically unenforceable due to vague language. Therefore, these states are not included as having sexual orientation hate crimes legislation (NGLTF, 1998).
} 
for the time period under consideration. However, a control variable is included in the analysis for the liberalness of a state, and this control is likely correlated with the prevalence of hate crimes. The presence of a hate crime law is expected to increase the in-migration and decrease the out-migration of gays and lesbians as it is expected to reduce the prevalence of hate crimes.

Sexual orientation private employment anti-discrimination laws prohibit discrimination in hiring, firing, and pay in private employment based on a person's sexual orientation. A private employment anti-discrimination law had been passed by eight states as of 1994 . The effect of this law on the employment outcomes of gays and lesbians is not evident a priori. The reason for this is that a private employment anti-discrimination law reduces the costs of hiring and increases the costs of firing gays and lesbians to the firm, as it increases the probability of the firm facing a lawsuit in each of these situations. A reduction in the costs of hiring gays and lesbians increases the labor demand for them, while an increase in the costs of firing gays and lesbians reduces the labor demand for them. Interacting with these effects is the equal pay provision of the private employment anti-discrimination law which requires gays and lesbians to be paid equal to heterosexuals performing similar jobs. This provision acts as a minimum wage law by increasing wages and reducing employment if the wage of gays and lesbians falls below the wage of heterosexuals.

Although the expected effect of a private employment anti-discrimination law is theoretically ambiguous, empirical evidence exists regarding the effect of the law. Evidence provided by Klawitter and Flatt (1998) suggests that the existence of a private employment anti-discrimination law does not affect the wages of gays or lesbians. Additionally, chapter 2 of this dissertation provides evidence that suggests gays experience higher wages and lower employment, while lesbian employment outcomes are unaffected, by the passage of a private employment anti-discrimination law. Taking these studies into consideration, the expected impact of a private employment anti-discrimination law on the in-migration and out-migration of gays is ambiguous. For lesbians, the expectation is that the law has no 
impact on the migration.

Sexual orientation public employment anti-discrimination laws prohibit discrimination in hiring, firing, and pay in public employment based on a person's sexual orientation. A public employment anti-discrimination law had been passed by 13 states as of 1994. 7 The theoretical impact regarding employment outcomes of a public employment anti-discrimination law is the same as a private employment one since it includes identical provisions. Despite this, the public employment anti-discrimination law covers relatively few jobs in the economy. Therefore, it likely does not have an effect on gay and lesbian migration through its impact on employment outcomes. However, the law may still have an impact on migration, as gays and lesbians may receive utility from the existence of the law in itself. If this is the case, the existence of the public employment anti-discrimination law is expected to increase in-migration and reduce out-migration of gays and lesbians.

In fact, the possibility that gays and lesbians receive utility from the existence of a law in itself is a possibility for all of the pieces of legislation in this analysis. The reasoning for this possibility is that gays and lesbians likely receive negative utility from having to hide their sexual orientation in the work place and other facets of life. The presence of a particular gay and lesbian rights or protection law may make gay and lesbian individuals less stressed over hiding their sexual orientation, increasing their happiness (Badgett, 2001). In addition to each of the effects of the laws already mentioned above, if laws in themselves have value to gays and lesbians then this effect is expected to cause the presence of each law to increase the in-migration and decrease the out-migration of gays and lesbians.

Gays and lesbians are often thought of as choosing to live in locations that are more liberal. The likelihood exists that the liberalness of a location is correlated with the existence of gay and lesbian rights legislation. Therefore, the liberalness of a state must be controlled for in order to avoid an omitted variables bias in the coefficients on the law variables. Unfortunately, there isn't any obvious way to control for the liberalness of a state.

\footnotetext{
${ }^{7}$ States with executive orders prohibiting public employment sexual orientation discrimination are included as states with public employment anti-discrimination legislation.
} 
The approach taken in this paper is to use the percentage of the states population that voted for George Bush in the 1992 Presidential Election as a proxy for state liberalness as is done in Haider-Markel and Meier (1996) and Gates (2007). This proxy should adequately control for liberalness because George Bush ran a campaign based on strong family values in $1992 .^{8}$

The other explanatory variables that are included in this analysis are controls for economic opportunity, amenities, and cost of living at the origin and destination state that have typically been found in migration studies. These variables also take on their 1994 values, unless otherwise noted, in order to avoid issues with endogeneity. Median household income, employment growth, and the unemployment rate are included to account for economic opportunity. The specification of climate amenities is based on Cushing (1987), which suggests that extreme temperatures and other climate variables that may be correlated with temperature should be included in the regression. The variables that are therefore included to control for climate are cooling degree days, heating degree days, average wind speed, average percent relative humidity, and average days of sunshine. Additionally, controls are included for the non-climate amenities of the crime rate, existence of a coastline, percent of land cover by water, cost of living, and percent of land that is a part of state parks. Table 3.4 lists and defines all the variables used in this analysis along with the sources of those variables.

\footnotetext{
${ }^{8}$ Another variable that has been suggested to control for the attitude towards gays and lesbians is the percent of the state population that adheres to a religion or regularly attends church. Religion is not used in the main regression since there is ambiguity concerning which religious denominations do or do not accept gays and lesbians. State level survey data from 1990 provided by ARDA on religious adherents is used in place of the Bush variable in robustness checks and this variable is insignificant and its use does not significantly change the reported results. Black et al. (2002) uses individual survey responses in the General Social Survey to questions regarding homosexuality to control for public attitude. Unfortunately, the smallest geographic unit that can be identified on the publicly available GSS data is the region (GSS, 2010).
} 


\section{$5 \quad$ Results}

\subsection{Descriptive Statistics}

The data on migration flows from table 3.1 can be used to gauge the appropriateness of the gravity model in analyzing gay and lesbian migration. The states that were most often the largest recipient of out-migrants from another state from 1995-2000 were California (13), Florida (5), Georgia (3), Minnesota (3), Texas (4), and Washington (3). The states that were most often the largest source of in-migrants for another state were California (13), New York (6), Texas (5), and Massachusetts (4). From this data, population clearly plays a significant role in gay and lesbian migration flows, but it also appears that population is not the only determinant of migration. For example, although New York had the second largest gay and lesbian population at the start of the period and was the largest source of in-migrants for six states, it was not the largest recipient of in-migrants for any state. Table 3.1 also shows that distance plays an important role in gay and lesbian migration as many of the top out-migrant and in-migrant flows for states are with states that are in close proximity to them.

The data on net-migration in table 3.2 and on the presence of state laws in table 3.3 can be used to develop an idea on the possible effect of gay and lesbian legislation on migration. $25 \%$ of the eight states that had passed a private employment anti-discrimination law by 1994 had a positive net-migration, while $37 \%$ of the 41 states that did not pass this legislation experienced a positive net-migration. Also, of the 13 states that passed a public employment anti-discrimination law, $31 \%$ had a positive net-migration, while $36 \%$ that did not pass a law had a positive net-migration. These statistics suggest that employment anti-discrimination laws may have a negative impact on gays and lesbians. As for the hate crime law, $44 \%$ of the 16 states that passed a law had a positive net-migration, while $30 \%$ of the 33 states that did not pass a law experienced a positive net-migration. This result for the hate crime law

indicates that gays and lesbians may benefit from the existence of this law. Lastly, none of the three states that had a domestic partnership law as of 1994 had positive net-migration, 
suggesting that this law may not matter or may have a negative impact on gays and lesbians.

\subsection{Main Model Specification}

The direct effect results from the estimation of the spatial autoregressive Tobit model are reported in column 2 of tables 3.5 and 3.6 . The estimate for the spatial dependence coefficient $\rho$ is .12. Although this coefficient is relatively small, it is positive and statistically significant indicating that there is positive spatial dependence in the gay and lesbian migration flows. This result provides evidence that a spatial model for analyzing gay and lesbian migration flows is superior to the non-spatial model that has been historically used to analyze migration. The results for the coefficients of the standard gravity model variables of population and distance are as predicted by standard gravity model theory. Distance between the origin state and destination state has a statistically significant negative effect on the state-tostate migration flow that decreases with distance. The coefficients on both the origin and destination population variables are positive and statistically significant.

A feature of the remaining results is that a number of the origin and destination coefficients exhibit the same sign. This result appears to be puzzling for most variables since standard utility maximization theory suggests that variables have the opposite impact on in-migration as they do on out-migration. The same-sign issue is not unique to this paper as it is present in elderly migration work and multiple other migration studies. An explanation commonly put forth concerning this issue is that asymmetries in the origin and destination variables are likely to exist. For instance, an individual may not have full information of the attributes of the destination or may discount the influence of variables there such as crime.

The results for the amenity variables are generally as expected. These results indicate that gays and lesbians migrate into places that are not extremely cold, that have a coastline, and that are more liberal. They migrate away from places that are extremely cold and have

high average wind speeds. The one result that goes against expectations is that an increase in percentage sunshine leads to an increase in out-migration. This result might be explained 
by collinearity with the cooling degree days variables which have the expected signs but are statistically insignificant. Neither the origin or destination variables for the percentage of land covered by water, percentage of land devoted to state parks, crime rate, humidity, cost of living, or cooling degree days have statistically significant coefficients, suggesting these amenities do not factor into the utility of gays and lesbians.

As for the economic opportunity variables, the unemployment rate appears to be the most important of these variables influencing gay and lesbian migration. Specifically, an increase in the unemployment rate causes a decrease in in-migration to a state. Employment growth leads to an increase in in-migration as expected, but also causes an increase in outmigration. This same-sign issue for employment growth can likely be explained by places with high employment growth being vibrant places with people often moving in and out. The coefficients on both the origin and destination median household income variables are statistically insignificant. This indicates that after controlling for amenities and cost of living median household income does not factor into gay and lesbian migration.

The coefficients mainly of interest are those on the legislation variables. The evidence suggests that the existence of a private employment anti-discrimination law does not have an impact on out-migration, but causes a reduction in in-migration. This result indicates that a private employment anti-discrimination law has a negative impact on gay and lesbian employment outcomes that outweighs any positive effect that might exist from the existence of the law. As for the public employment anti-discrimination and hate crime laws, the existence of these laws also do not have an impact on out-migration, but result in an increase in-migration. These results suggest that gays and lesbians gain utility from living in places that have passed a public employment anti-discrimination or a hate crime law. Lastly, it is found that the existence of a domestic partnership does not have an impact on in-migration or out-migration, indicating that this law may not have an effect on the utility of gays and lesbians.

For comparison, reported in column 1 of tables 3.5 and 3.6 are the results from the estima- 
tion of the Tobit model excluding the spatial weight matrix. The statistical significance and sign of the legislation coefficients is unchanged between the two models, with the exception of the hate crime in-migration law becoming statistically significant at the $5 \%$ significance level. A priori, the magnitude of the coefficients for the law variables of the spatial model could be greater or less than those of the non-spatial model. The coefficients are expected to be less than those of the non-spatial model because the non-spatial model attributes some of the spatial correlation in migration flows to the presence of laws. On the other hand, the spatial model coefficients include not only the initial impact of a change in a law on a migration flow, but also the feedback that occurs when that migration flow impacts other migration flows that then feed back onto the initial flow. In looking at the statistically significant coefficients on the law variables, the coefficient on the non-spatial private employment anti-discrimination in-migration variable is higher (in absolute value), the coefficient on the non-spatial public employment anti-discrimination in-migration variable is higher, and the coefficient on the non-spatial hate crime variable is approximately the same as the spatial coefficient.

\subsection{Checks for Robustness}

One possible issue with the results reported up to this point is that only states with a particular law as of 1994 have been considered as having that law in existence. The possibility exists that laws passed at the beginning of the migration period could also significantly influence migration decisions over the time period. The reason for this is that a number of the migration moves are likely to of occurred in years after the beginning of the migration period, in which case those migrants would be basing their decisions on laws passed in the year 1995 or 1996. Therefore, the model is re-estimated first using 1995 as the cutoff year

for legislation and then using 1996 as the cutoff year to check for robustness of the results.

In column 3 of tables 3.5 and 3.6 coefficients are reported for the estimation of the model using 1995 as the cutoff year, and in column 4 coefficients are reported for estimation of 
the model using 1996 as the cutoff year. For the most part, the results for the legislation variables are similar to the results obtained when 1994 is used as the cutoff year. The one major change when 1995 or 1996 is used as the cutoff year is that the private employment anti-discrimination law is now indicated as reducing out-migration from a state. Therefore, the coefficient on this variable now exhibits the same-sign issue as it also indicates that a private employment law decreases in-migration to a state. This phenomenon can likely be explained by two competing influences on gays and lesbians. For one, they may see the law as being a positive protection of their rights and gain utility from the existence of the law in itself. At the same time, it is harder for them to find employment in a state because of the existence of a law and this makes it less likely for them to move there.

Another possible issue is that legislation targeted towards gays and lesbians may have a different effect on gays than on lesbians. Tebaldi and Elmslie (2006) provides evidence that gays face labor market discrimination and that lesbians do not. Also, results from Herek (2009) show that gays are likelier to have hate crimes committed against them than lesbians are. Additionally, chapter 2 of this dissertation provides evidence that the passage of a private employment anti-discrimination law reduces employment and raises wages for gays and it does not have an effect on labor market outcomes for lesbians. In order to account for this possibility, the sample is separated into separate gay and lesbian migration flows and is re-estimated for both of these samples.

Separate results for the gay and lesbian samples are reported in tables 3.7 and 3.8 . The results are reported using 1994, 1995, and 1996 as the cutoff year for legislation. For both the gay and lesbian samples, the private employment anti-discrimination law reduces in-migration for all three cutoff year specifications. Also, a hate crime law increases inmigration in all specifications, with the exception of the 1994 cutoff year for the gay sample. The finding that lesbians are negatively impacted by private employment anti-discrimination laws in addition to gays doesn't seem to match with previous research, but these laws are often passed along with other anti-discrimination measures that could negatively impact 
lesbians in similar fashion as the private employment laws do for gays.

One other possible issue is that only state level laws have been considered up to this point. As of 1994, local governments had also passed legislation in regards to domestic partnerships and public and private employment discrimination. There are two main reasons for only considering state laws and ignoring local gay and lesbian rights legislation. One reason is that local laws vary widely from place to place in their enforceability based on, and this enforceability is not revealed through the data on the existence of these laws. Also, the survey results suggesting that gays and lesbians lack knowledge on basic gay and lesbian rights legislation at the national and state levels would seem to also suggest that they would be even more likely to lack knowledge on the often less publicized local laws. However, there is the possibility that local legislation that has been relegated to the error term has an effect on gays and lesbians and is therefore impacting the results that have been reported so far.

The problem that arises is finding a way to incorporate local legislation into a state-tostate migration flows model. The method that is used in Haider-Markel and Meier (1996) to incorporate local legislation into a state level model of legislation adoption is to use the percentage of the state's population that is covered by either a local or state law as the legislation variable. This variable represents the probability of an individual being covered by a particular law by residing in a state. The three gay and lesbian rights laws that vary at the local level and that are constructed in this way are the private employment antidiscrimination, public employment anti-discrimination, and domestic partnership law. Hate crime laws only vary at the state level and are kept as a binary variable representing if a state had a hate crime law or not.

The results for the re-estimation of the model using this new specification of the legislation variables is reported in tables 3.9 and 3.10 for the full, gay, and lesbian samples. The results for the coefficient sign and statistical significance of the private employment in-migration and hate crime law in-migration variables are similar to those results in the state laws regressions. As for domestic partnership laws, they vary much more at the local than at the state level of 
government, so it might be expected that if these laws matter to gays and lesbians then this might show up when information on local laws is included into the analysis. Despite this, the coefficients on both the in-migration and out-migration domestic partnership variables continue to be statistically insignificant.

\section{Conclusion}

The results of this chapter provide evidence that the passage of certain types of gay and lesbian rights legislation do have an impact on the utility of gays and lesbians. The estimation of the main model specification using 1994 as the cutoff year for the passage of legislation shows that public employment anti-discrimination laws and hate crime laws increase inmigration, while private employment anti-discrimination laws decrease in-migration. These results indicate that gays and lesbians are made better off as the result of the passage of public employment anti-discrimination and hate crime laws, and that they are made worse off by the passage of private employment anti-discrimination laws. Robustness checks that switch the cutoff year for the data on the passage of legislation to 1995 and 1996 suggest that private employment laws may also have a negative impact on out-migration, which suggest that gays and lesbians actually benefit from these laws. This same-sign issue with the private employment law can likely be explained by the law having a negative impact on gay and lesbian employment outcomes while at the same time benefiting them as they feel more welcomed in these places.

These results have significance at the federal level of government. For one, they suggest that the recently passed federal law including sexual orientation into the national hate crimes law has increased the utility of gays and lesbians. The results also indicate that the effect of the passage of the federal private sector anti-discrimination law that is being currently considered in Congress is not clear. The evidence provided on the impact of private employment anti-discrimination on the employment of gays provided in the last chapter, and on the 
impact of this law on in-migration provided in this chapter, suggest a negative effect of this law on gays and lesbians. The possible reduction in out-migration also found in this chapter, as well as the increases in wages for gays found in the last chapter, indicate a positive impact of this legislation. What these results do suggest is that a more in depth discussion and further research needs to take place before this legislation is passed at the federal level. These results also have policy implications for states that may want to pass legislation in order to attract gays and lesbians for economic growth purposes. In particular, the findings of this chapter unambiguously indicate that states that want to attract gays and lesbians should pass public employment anti-discrimination laws. This finding is important as there are likely to be large changes in gay and lesbian populations at stake with the passage or nonpassage of legislation. Estimates of the total homosexual population from previous studies range from $4 \%$ to $10 \%$ of the overall population, and the homosexual population is likely to be relatively mobile as these individuals are less likely constrained by children and other family obligations. 
Table 3.1: Source and Magnitude of Top Gay and Lesbian Migrant In-flow and Out-Flow By State

\begin{tabular}{|c|c|c|c|c|c|}
\hline State & Population & Top Out Flow To: & Flow Size & Top In Flow From: & Flow Size \\
\hline Alabama & 11604 & Florida & 277 & Georgia & 271 \\
\hline Arizona & 17419 & California & 936 & California & 728 \\
\hline Arkansas & 5080 & Texas & 306 & California & 145 \\
\hline California & 151615 & Washington & 1723 & New York & 1644 \\
\hline Colorado & 16679 & California & 545 & Texas & 467 \\
\hline Connecticut & 11780 & Florida & 253 & New York & 336 \\
\hline Delaware & 2164 & Maryland & 145 & Pennsylvania & 240 \\
\hline District of Columbia & 5865 & Virginia & 479 & Maryland & 509 \\
\hline Florida & 54980 & Georgia & 1220 & New York & 1413 \\
\hline Georgia & 30051 & Florida & 591 & Florida & 1220 \\
\hline Idaho & 3053 & Washington & 87 & Nevada & 90 \\
\hline Illinois & 35508 & California & 768 & California & 576 \\
\hline Indiana & 17617 & Kentucky & 364 & Illinois & 288 \\
\hline Iowa & 6495 & California & 161 & Minnesota & 150 \\
\hline Kansas & 6399 & Missouri & 249 & Missouri & 327 \\
\hline Kentucky & 9594 & Ohio & 240 & Indiana & 364 \\
\hline Louisiana & 15567 & Texas & 348 & Mississippi & 175 \\
\hline Maine & 4601 & Florida & 143 & Massachusetts & 288 \\
\hline Maryland & 18155 & $\mathrm{DC}$ & 509 & Virginia & 487 \\
\hline Massachusetts & 29121 & California & 631 & New York & 520 \\
\hline Michigan & 24212 & California & 310 & California & 279 \\
\hline Minnesota & 14296 & California & 277 & Wisconsin & 440 \\
\hline Mississippi & 7428 & Tennessee & 207 & California & 134 \\
\hline Missouri & 15361 & Kansas & 327 & Kansas & 249 \\
\hline Montana & 2690 & California & 219 & Texas & 48 \\
\hline Nebraska & 4211 & Colorado & 133 & Iowa & 132 \\
\hline Nevada & 6578 & California & 205 & California & 1356 \\
\hline New Hampshire & 5054 & Massachusetts & 272 & Massachusetts & 278 \\
\hline New Jersey & 25941 & Pennsylvania & 857 & New York & 1243 \\
\hline New Mexico & 6186 & Texas & 243 & California & 152 \\
\hline New York & 75805 & California & 1644 & California & 888 \\
\hline North Carolina & 24931 & Georgia & 617 & New York & 683 \\
\hline North Dakota & 927 & Minnesota & 71 & Minnesota & 97 \\
\hline Ohio & 30631 & Florida & 622 & Michigan & 262 \\
\hline Oklahoma & 8020 & Texas & 387 & Texas & 344 \\
\hline Oregon & 14248 & Washington & 473 & California & 615 \\
\hline Pennsylvania & 34470 & New Jersey & 647 & New Jersey & 857 \\
\hline Rhode Island & 4263 & Massachusetts & 280 & Massachusetts & 173 \\
\hline South Carolina & 11658 & Georgia & 291 & North Carolina & 222 \\
\hline South Dakota & 1303 & Minnesota & 285 & Nebraska & 69 \\
\hline Tennessee & 15720 & Kentucky & 218 & Texas & 337 \\
\hline Texas & 66297 & California & 1087 & California & 1025 \\
\hline Utah & 5460 & California & 229 & California & 200 \\
\hline Vermont & 3456 & New Hampshire & 109 & Massachusetts & 164 \\
\hline Virginia & 23069 & Maryland & 487 & DC & 479 \\
\hline Washington & 25494 & California & 554 & California & 1723 \\
\hline West Virginia & 5425 & Virginia & 137 & California & 73 \\
\hline Wisconsin & 14262 & Minnesota & 440 & Illinois & 285 \\
\hline Wyoming & 1212 & Colorado & 120 & Texas & 49 \\
\hline
\end{tabular}

${ }^{a}$ Gay and lesbian population and migration flows are calculated from the 5\% PUMS of the 2000 Census. The 5\% PUMS of the 2000 Census is available from www.ipums.org. 
Table 3.2: Out, In, and Net Gay and Lesbian Migration by State

\begin{tabular}{|c|c|c|c|c|}
\hline State & Population & Out-Migrants & In-Migrants & Net-Migrants \\
\hline Alabama & 11604 & 1456 & 1452 & -4 \\
\hline Arizona & 17419 & 2348 & 4215 & 1867 \\
\hline Arkansas & 5080 & 951 & 902 & -49 \\
\hline California & 151615 & 12961 & 12944 & -17 \\
\hline Colorado & 16679 & 2317 & 3632 & 1315 \\
\hline Connecticut & 11780 & 1326 & 1364 & 38 \\
\hline Delaware & 2164 & 327 & 813 & 486 \\
\hline District of Columbia & 5865 & 1916 & 1479 & -437 \\
\hline Florida & 54980 & 6362 & 9564 & 3202 \\
\hline Georgia & 30051 & 3938 & 6281 & 2343 \\
\hline Idaho & 3053 & 494 & 390 & -104 \\
\hline Illinois & 35508 & 4367 & 3766 & -601 \\
\hline Indiana & 17617 & 2421 & 1750 & -671 \\
\hline Iowa & 6495 & 1084 & 633 & -451 \\
\hline Kansas & 6399 & 1188 & 1487 & 299 \\
\hline Kentucky & 9594 & 1203 & 1715 & 512 \\
\hline Louisiana & 15567 & 2022 & 1320 & -702 \\
\hline Maine & 4601 & 762 & 1136 & 374 \\
\hline Maryland & 18155 & 2811 & 2645 & -166 \\
\hline Massachusetts & 29121 & 4330 & 3349 & -981 \\
\hline Michigan & 24212 & 2585 & 2117 & -468 \\
\hline Minnesota & 14296 & 1805 & 2403 & 598 \\
\hline Mississippi & 7428 & 852 & 819 & -33 \\
\hline Missouri & 15361 & 2347 & 1528 & -819 \\
\hline Montana & 2690 & 806 & 215 & -591 \\
\hline Nebraska & 4211 & 882 & 432 & -450 \\
\hline Nevada & 6578 & 975 & 2532 & 1557 \\
\hline New Hampshire & 5054 & 928 & 672 & -256 \\
\hline New Jersey & 25941 & 3733 & 3060 & -673 \\
\hline New Mexico & 6186 & 1058 & 936 & -122 \\
\hline New York & 75805 & 9473 & 5979 & -3494 \\
\hline North Carolina & 24931 & 2951 & 4044 & 1093 \\
\hline North Dakota & 927 & 155 & 107 & -48 \\
\hline Ohio & 30631 & 4402 & 2489 & -1913 \\
\hline Oklahoma & 8020 & 1513 & 1146 & -367 \\
\hline Oregon & 14248 & 1800 & 2416 & 616 \\
\hline Pennsylvania & 34470 & 3682 & 2985 & -697 \\
\hline Rhode Island & 4263 & 838 & 565 & -273 \\
\hline South Carolina & 11658 & 1474 & 1260 & -214 \\
\hline South Dakota & 1303 & 197 & 277 & 80 \\
\hline Tennessee & 15720 & 2257 & 2723 & 466 \\
\hline Texas & 66297 & 7007 & 7290 & 283 \\
\hline Utah & 5460 & 867 & 859 & -8 \\
\hline Vermont & 3456 & 551 & 505 & -46 \\
\hline Virginia & 23069 & 4328 & 3280 & -1048 \\
\hline Washington & 25494 & 2941 & 4757 & 1816 \\
\hline West Virginia & 5425 & 658 & 123 & -535 \\
\hline Wisconsin & 14262 & 1799 & 1266 & -533 \\
\hline Wyoming & 1212 & 403 & 229 & -174 \\
\hline
\end{tabular}

${ }^{\text {a }}$ Gay and lesbian population and migration flows are calculated from the $5 \%$ PUMS of the 2000 Census. The $5 \%$ PUMS of the 2000 Census is available from www.ipums.org. 
Table 3.3: Year of Passage of Legislation for States With a Law as of 1996

\begin{tabular}{|c|c|c|c|c|}
\hline State & Private & Public & Hate Crime & Partnership \\
\hline Alabama & $\mathrm{x}$ & $\mathrm{x}$ & $\mathrm{x}$ & $\mathrm{x}$ \\
\hline Arizona & $\mathrm{x}$ & $\mathrm{x}$ & $\mathrm{x}$ & $\mathrm{x}$ \\
\hline Arkansas & $\mathrm{x}$ & $\mathrm{x}$ & $\mathrm{x}$ & $\mathrm{x}$ \\
\hline California & 1990 & 1979 & 1984 & $\mathrm{x}$ \\
\hline Colorado & & 1990 & $\mathrm{x}$ & $\mathrm{x}$ \\
\hline Connecticut & 1991 & 1991 & 1987 & $\mathrm{x}$ \\
\hline Delaware & $\mathrm{x}$ & $\mathrm{x}$ & 1995 & $\mathrm{x}$ \\
\hline District of Columbia & 1991 & 1977 & 1977 & 1992 \\
\hline Florida & $\mathrm{x}$ & $\mathrm{x}$ & 1991 & $\mathrm{x}$ \\
\hline Georgia & $\mathrm{x}$ & $\mathrm{x}$ & $\mathrm{x}$ & $\mathrm{x}$ \\
\hline Idaho & $\mathrm{x}$ & $\mathrm{x}$ & $\mathrm{x}$ & $\mathrm{x}$ \\
\hline Illinois & $\mathrm{x}$ & 1996 & 1990 & $\mathrm{x}$ \\
\hline Indiana & $\mathrm{x}$ & $\mathrm{x}$ & $\mathrm{x}$ & $\mathrm{x}$ \\
\hline Iowa & $\mathrm{x}$ & $\mathrm{x}$ & 1990 & $\mathrm{x}$ \\
\hline Kansas & $\mathrm{x}$ & $\mathrm{x}$ & $\mathrm{x}$ & $\mathrm{x}$ \\
\hline Kentucky & $\mathrm{x}$ & $\mathrm{x}$ & $\mathrm{x}$ & $\mathrm{x}$ \\
\hline Louisiana & $\mathrm{x}$ & $\mathrm{x}$ & $\mathrm{x}$ & $\mathrm{x}$ \\
\hline Maine & $\mathrm{x}$ & $\mathrm{x}$ & 1987 & $\mathrm{x}$ \\
\hline Maryland & $\mathrm{x}$ & 1995 & $\mathrm{x}$ & $\mathrm{x}$ \\
\hline Massachusetts & 1989 & 1989 & 1996 & 1992 \\
\hline Michigan & $\mathrm{x}$ & $\mathrm{x}$ & $\mathrm{x}$ & $\mathrm{x}$ \\
\hline Minnesota & 1993 & 1993 & 1988 & $\mathrm{x}$ \\
\hline Mississippi & $\mathrm{x}$ & $\mathrm{x}$ & $\mathrm{x}$ & $\mathrm{x}$ \\
\hline Missouri & $\mathrm{x}$ & $\mathrm{x}$ & $\mathrm{x}$ & $\mathrm{x}$ \\
\hline Montana & $\mathrm{x}$ & $\mathrm{x}$ & $\mathrm{x}$ & $\mathrm{x}$ \\
\hline Nebraska & $\mathrm{x}$ & $\mathrm{x}$ & $\mathrm{x}$ & $\mathrm{x}$ \\
\hline Nevada & $\mathrm{x}$ & $\mathrm{x}$ & 1989 & $\mathrm{x}$ \\
\hline New Hampshire & $\mathrm{x}$ & $\mathrm{x}$ & 1990 & $\mathrm{x}$ \\
\hline New Jersey & 1992 & 1992 & 1990 & $\mathrm{x}$ \\
\hline New Mexico & $\mathrm{x}$ & $\mathrm{x}$ & $\mathrm{x}$ & $\mathrm{x}$ \\
\hline New York & $\mathrm{x}$ & 1994 & $\mathrm{x}$ & $\mathrm{x}$ \\
\hline North Carolina & $\mathrm{x}$ & $\mathrm{x}$ & $\mathrm{x}$ & $\mathrm{x}$ \\
\hline North Dakota & $\mathrm{x}$ & $\mathrm{x}$ & $\mathrm{x}$ & $\mathrm{x}$ \\
\hline Ohio & $\mathrm{x}$ & 1992 & $\mathrm{x}$ & $\mathrm{x}$ \\
\hline Oklahoma & $\mathrm{x}$ & $\mathrm{x}$ & $\mathrm{x}$ & $\mathrm{x}$ \\
\hline Oregon & $\mathrm{x}$ & $\mathrm{x}$ & 1989 & $\mathrm{x}$ \\
\hline Pennsylvania & $\mathrm{x}$ & 1988 & $\mathrm{x}$ & $\mathrm{x}$ \\
\hline Rhode Island & 1995 & 1995 & 1991 & $\mathrm{x}$ \\
\hline South Carolina & $\mathrm{x}$ & $\mathrm{x}$ & $\mathrm{x}$ & $\mathrm{x}$ \\
\hline South Dakota & $\mathrm{x}$ & $\mathrm{x}$ & $\mathrm{x}$ & $\mathrm{x}$ \\
\hline Tennessee & $\mathrm{x}$ & $\mathrm{x}$ & $\mathrm{x}$ & $\mathrm{x}$ \\
\hline Texas & $\mathrm{x}$ & $\mathrm{x}$ & $\mathrm{x}$ & $\mathrm{x}$ \\
\hline Utah & $\mathrm{x}$ & $\mathrm{x}$ & $\mathrm{x}$ & $\mathrm{x}$ \\
\hline Vermont & 1993 & 1993 & 1990 & 1991 \\
\hline Virginia & $\mathrm{x}$ & $\mathrm{x}$ & $\mathrm{x}$ & $\mathrm{x}$ \\
\hline Washington & $\mathrm{x}$ & 1985 & 1993 & $\mathrm{x}$ \\
\hline West Virginia & $\mathrm{x}$ & $\mathrm{x}$ & $\mathrm{x}$ & $\mathrm{x}$ \\
\hline Wisconsin & 1978 & 1978 & 1988 & $\mathrm{x}$ \\
\hline Wyoming & $\mathrm{x}$ & $\mathrm{x}$ & $\mathrm{x}$ & $\mathrm{x}$ \\
\hline
\end{tabular}

${ }^{a}$ Years in bold represent laws passed as of 1994. These are the laws used in the main regression specification. The data on laws is from NGLTF (1998) and Rimmerman et al., eds (2000]. 
Table 3.4: Definition and Source of Variables

\begin{tabular}{|c|c|c|}
\hline Variable & Definition & Source \\
\hline Migration Flow & $\begin{array}{l}\text { origin to destination state migration flow } \\
\text { of gays and lesbians between } 1995-2000\end{array}$ & $\begin{array}{l}\text { Author's Calculation from } \\
2000 \text { U.S. Census 5\% PUMS }\end{array}$ \\
\hline Distance & $\begin{array}{l}\text { distance in hundreds of miles between the geographic } \\
\text { center of each origin and destination state }\end{array}$ & $\begin{array}{l}\text { Author's Calculation } \\
\text { Using MATLAB 7.10.0 }\end{array}$ \\
\hline Population & $\begin{array}{l}\text { estimate of gay and lesbian population at } \\
\text { the beginning of the migration period }\end{array}$ & $\begin{array}{l}\text { Author's Calculation from } \\
2000 \text { U.S. Census 5\% PUMS }\end{array}$ \\
\hline Employment Growth & $\begin{array}{l}\text { percentage change in } \\
\text { employment from } 1990 \text { to } 1994\end{array}$ & $\begin{array}{l}\text { Statistical Abstract of the } \\
\text { United States }\end{array}$ \\
\hline Income & median household income in 1994 & $\begin{array}{l}\text { Statistical Abstract of the } \\
\text { United States }\end{array}$ \\
\hline Unemployment & unemployment rate in 1994 & $\begin{array}{l}\text { Statistical Abstract of the } \\
\text { United States }\end{array}$ \\
\hline Crime Rate & $\begin{array}{l}\text { number of crimes committed } \\
\text { per 100,000 people in } 1994\end{array}$ & $\begin{array}{l}\text { Statistical Abstract of the } \\
\text { United States }\end{array}$ \\
\hline Cooling & $\begin{array}{l}\text { population weighted average number of } \\
\text { annual cooling degree days }\end{array}$ & $\begin{array}{l}\text { National Oceanic and } \\
\text { Atmosphere Administration }\end{array}$ \\
\hline Heating & $\begin{array}{l}\text { population weighted average number of } \\
\text { annual heating degree days }\end{array}$ & $\begin{array}{l}\text { National Oceanic and } \\
\text { Atmosphere Administration }\end{array}$ \\
\hline Sunshine & $\begin{array}{l}\text { average percentage days of } \\
\text { sunshine annually }\end{array}$ & $\begin{array}{l}\text { Statistical Abstract of the } \\
\text { United States }\end{array}$ \\
\hline Wind & annual average wind speed in miles per hour & $\begin{array}{l}\text { Statistical Abstract of the } \\
\text { United States }\end{array}$ \\
\hline Humidity & annual average percent relative humidity & $\begin{array}{l}\text { Statistical Abstract of the } \\
\text { United States }\end{array}$ \\
\hline Water Cover & percent of state land covered by lakes and rivers & $\begin{array}{l}\text { Statistical Abstract of the } \\
\text { United States }\end{array}$ \\
\hline State Park & percent of state land that is part of a state park & $\begin{array}{l}\text { Statistical Abstract of the } \\
\text { United States }\end{array}$ \\
\hline Cost of Living & $\begin{array}{l}\text { cost of living index created by (CITE) for } 1990 \\
\text { and adjusted for } 1994 \text { using the state GDP deflator }\end{array}$ & $\begin{array}{l}\text { CITE and } \\
\text { Bureau of Labor Statistics }\end{array}$ \\
\hline Coastline & $\begin{array}{l}\text { binary variable equaling one if there } \\
\text { is an ocean or Great Lake coastline }\end{array}$ & $\begin{array}{l}\text { Statistical Abstract of the } \\
\text { United States }\end{array}$ \\
\hline Bush Vote & $\begin{array}{l}\text { percentage of the state vote that George Bush } \\
\text { received in the } 1992 \text { President election }\end{array}$ & www.uselectionatlas.org \\
\hline Private Law & $\begin{array}{l}\text { binary variable equaling on if the state had a private } \\
\text { employment anti-discrimination law as of the cutoff year }\end{array}$ & NGLTF Policy Institute \\
\hline Hate Crime Law & $\begin{array}{l}\text { binary variable equaling one if the state had a } \\
\text { hate crime law as of the cutoff year }\end{array}$ & NGLTF Policy Institute \\
\hline Partnership Law & $\begin{array}{l}\text { binary variable equaling one if the state had a } \\
\text { domestic partnership law as of the cutoff year }\end{array}$ & NGLTF Policy Institute \\
\hline Public Law & $\begin{array}{l}\text { binary variable equaling one if the state had a public } \\
\text { employment anti-discrimination law as of the cutoff year }\end{array}$ & NGLTF Policy Institute \\
\hline
\end{tabular}


Table 3.5: Full Sample Bayesian Spatial Autoregressive Tobit Model Estimation Results

\begin{tabular}{|c|c|c|c|c|}
\hline & $\begin{array}{c}(1) \\
\text { Non-Spatial }\end{array}$ & $\begin{array}{c}(2) \\
1994 \text { Cutoff }\end{array}$ & $\begin{array}{c}(3) \\
1995 \text { Cutoff }\end{array}$ & $\begin{array}{c}(4) \\
1996 \text { Cutoff }\end{array}$ \\
\hline Stayer & $\begin{array}{l}-27.86^{* * *} \\
(5.44)\end{array}$ & $\begin{array}{l}-27.69^{* * *} \\
(-5.64)\end{array}$ & $\begin{array}{l}-32.07^{* * *} \\
(-6.43)\end{array}$ & $\begin{array}{l}-31.02^{* * *} \\
(-6.20)\end{array}$ \\
\hline Mover & $\begin{array}{l}-32.24^{* * *} \\
(5.46)\end{array}$ & $\begin{array}{l}-32.43^{* * *} \\
(-6.68)\end{array}$ & $\begin{array}{l}-36.83^{* * *} \\
(-7.42)\end{array}$ & $\begin{array}{l}-35.75^{* * *} \\
(-7.15)\end{array}$ \\
\hline Distance & $\begin{array}{l}-0.7919^{* * *} \\
(0.0470)\end{array}$ & $\begin{array}{l}-0.6604^{* * *} \\
(-12.92)\end{array}$ & $\begin{array}{l}-0.6653^{* * *} \\
(-12.67)\end{array}$ & $\begin{array}{l}-0.6660^{* * *} \\
(-14.02)\end{array}$ \\
\hline Distance Squared & $\begin{array}{l}0.0248^{* * *} \\
(0.0020)\end{array}$ & $\begin{array}{l}0.0212^{* * *} \\
(10.40)\end{array}$ & $\begin{array}{l}0.0213^{* * *} \\
(10.13)\end{array}$ & $\begin{array}{l}0.0213^{* * *} \\
(11.31)\end{array}$ \\
\hline Log Population O & $\begin{array}{c}1.96^{* * *} \\
(0.1330)\end{array}$ & $\begin{array}{c}1.83^{* * *} \\
(14.37)\end{array}$ & $\begin{array}{c}1.92^{* * *} \\
(14.37)\end{array}$ & $\begin{array}{l}1.95^{\text {*** }} \\
(14.66)\end{array}$ \\
\hline Log Population D & $\begin{array}{c}1.78^{* * *} \\
(0.1331)\end{array}$ & $\begin{array}{l}1.37^{* * * *} \\
(10.61)\end{array}$ & $\begin{array}{l}1.54^{* * * *} \\
(11.81)\end{array}$ & $\begin{array}{l}1.53^{\text {*** }} \\
(11.45)\end{array}$ \\
\hline Cooling $\mathrm{O}$ & $\begin{array}{r}0.0002 \\
(0.0002)\end{array}$ & $\begin{array}{r}0.0003 \\
(1.31)\end{array}$ & $\begin{array}{r}0.0002 \\
(0.73)\end{array}$ & $\begin{array}{r}0.0002 \\
(0.74)\end{array}$ \\
\hline Cooling D & $\begin{array}{l}-0.0004^{* *} \\
(0.0002)\end{array}$ & $\begin{array}{r}-0.0003 \\
(-1.49)\end{array}$ & $\begin{array}{c}-0.0004^{*} \\
(-1.88)\end{array}$ & $\begin{array}{l}-0.0004^{* *} \\
(-1.96)\end{array}$ \\
\hline Heating $\mathrm{O}$ & $\begin{array}{l}0.0002^{* *} \\
(0.0001)\end{array}$ & $\begin{array}{l}0.0002^{* *} \\
(2.29)\end{array}$ & $\begin{array}{l}0.0002^{* *} \\
(2.29)\end{array}$ & $\begin{array}{l}0.0003^{* *} \\
(2.23)\end{array}$ \\
\hline Heating D & $\begin{array}{l}-0.0005^{* * *} \\
(0.0001)\end{array}$ & $\begin{array}{l}-0.0003^{* * *} \\
(-3.04)\end{array}$ & $\begin{array}{l}-0.0003^{* * *} \\
(-3.00)\end{array}$ & $\begin{array}{l}-0.0003^{* * *} \\
(-2.89)\end{array}$ \\
\hline Wind $\mathrm{O}$ & $\begin{array}{c}0.1708^{* * *} \\
(0.05680)\end{array}$ & $\begin{array}{l}0.1812^{* * *} \\
(3.19)\end{array}$ & $\begin{array}{l}0.2082^{* * *} \\
(3.52)\end{array}$ & $\begin{array}{l}0.1980^{* * *} \\
(3.20)\end{array}$ \\
\hline Wind D & $\begin{array}{r}0.0300 \\
(0.0602)\end{array}$ & $\begin{array}{r}0.0706 \\
(1.23)\end{array}$ & $\begin{array}{c}0.1035^{*} \\
(1.75)\end{array}$ & $\begin{array}{r}0.0837 \\
(1.42)\end{array}$ \\
\hline Sunshine O & $\begin{array}{l}0.0464^{* * *} \\
(0.0187)\end{array}$ & $\begin{array}{l}0.0485^{* * *} \\
(2.80)\end{array}$ & $\begin{array}{l}0.0548^{* * *} \\
(2.97)\end{array}$ & $\begin{array}{l}0.0498^{* * *} \\
(2.65)\end{array}$ \\
\hline Sunshine D & $\begin{array}{c}0.0397^{* *} \\
(0.0189)\end{array}$ & $\begin{array}{l}0.0403^{* *} \\
(2.28)\end{array}$ & $\begin{array}{l}0.0494^{* * *} \\
(2.69)\end{array}$ & $\begin{array}{l}0.0437^{* *} \\
(2.39)\end{array}$ \\
\hline Humidity $\mathrm{O}$ & $\begin{array}{r}0.0159 \\
(0.0193)\end{array}$ & $\begin{array}{r}0.0150 \\
(0.83)\end{array}$ & $\begin{array}{r}0.0178 \\
(0.94)\end{array}$ & $\begin{array}{r}0.0143 \\
(0.77)\end{array}$ \\
\hline Humidity D & $\begin{array}{l}-0.0278^{*} \\
(0.0192)\end{array}$ & $\begin{array}{r}-0.0202 \\
(-1.13)\end{array}$ & $\begin{array}{r}-0.0183 \\
(-1.01)\end{array}$ & $\begin{array}{r}-0.0216 \\
(-1.18)\end{array}$ \\
\hline Coastline O & $\begin{array}{c}-0.2444 \\
(0.2690)\end{array}$ & $\begin{array}{r}-0.1666 \\
(-0.61)\end{array}$ & $\begin{array}{r}-0.2497 \\
(-0.92)\end{array}$ & $\begin{array}{r}-0.2689 \\
(-0.89)\end{array}$ \\
\hline Coastline D & $\begin{array}{l}0.6680^{* * * *} \\
(0.2795)\end{array}$ & $\begin{array}{c}0.61370^{* *} \\
(2.26)\end{array}$ & $\begin{array}{c}0.4602^{*} \\
(1.68)\end{array}$ & $\begin{array}{r}0.3763 \\
(1.37)\end{array}$ \\
\hline Water Cover O & $\begin{array}{r}0.0109 \\
(0.0373)\end{array}$ & $\begin{array}{r}0.0165 \\
(0.45)\end{array}$ & $\begin{array}{r}0.0554 \\
(1.40)\end{array}$ & $\begin{array}{r}0.0565 \\
(1.40)\end{array}$ \\
\hline Water Cover D & $\begin{array}{r}0.0143 \\
(0.0387)\end{array}$ & $\begin{array}{r}0.0104 \\
(0.30)\end{array}$ & $\begin{array}{r}0.0556 \\
(1.37)\end{array}$ & $\begin{array}{r}0.0580 \\
(1.51)\end{array}$ \\
\hline State Park O & $\begin{array}{r}0.0045 \\
(0.0914)\end{array}$ & $\begin{array}{r}-0.0212 \\
(-0.23)\end{array}$ & $\begin{array}{r}-0.0416 \\
(-0.47)\end{array}$ & $\begin{array}{r}-0.0469 \\
(-0.51)\end{array}$ \\
\hline State Park D & $\begin{array}{r}0.01420 \\
(0.0866)\end{array}$ & $\begin{array}{r}-0.0249 \\
(-0.29)\end{array}$ & $\begin{array}{r}-0.0850 \\
(-0.98)\end{array}$ & $\begin{array}{r}-0.1176 \\
(-1.27)\end{array}$ \\
\hline
\end{tabular}

${ }^{a}$ Statistical significance is reported at the $1 \%(* * *), 5 \%(* *)$, and $10 \%\left({ }^{*}\right)$ levels.

${ }^{\mathrm{b}}$ Standard deviations are reported in parenthesis for the the non-spatial Tobit model.

${ }^{c}$ T-statistics are reported in parenthesis for the spatial Tobit models.

Continued on next page... 
Table 3.6: (Continued):Full Sample Bayesian Spatial Autoregressive Tobit Model Estimation Results

\begin{tabular}{|c|c|c|c|c|}
\hline & $\begin{array}{c}(1) \\
\text { Non-Spatial }\end{array}$ & $\begin{array}{c}(2) \\
1994 \text { Cutoff }\end{array}$ & $\begin{array}{c}(3) \\
1995 \text { Cutoff }\end{array}$ & $\begin{array}{c}(4) \\
1996 \text { Cutoff }\end{array}$ \\
\hline Crime Rate $\mathrm{O}$ & $\begin{array}{r}0.00007 \\
(0.00009)\end{array}$ & $\begin{array}{r}0.00002 \\
(0.23)\end{array}$ & $\begin{array}{r}-0.00002 \\
(-0.17)\end{array}$ & $\begin{array}{r}0.00000 \\
(0.03)\end{array}$ \\
\hline Crime Rate D & $\begin{array}{r}0.00008 \\
(0.00009)\end{array}$ & $\begin{array}{r}0.00009 \\
(0.99)\end{array}$ & $\begin{array}{r}0.00002 \\
(0.26)\end{array}$ & $\begin{array}{r}0.00004 \\
(0.49)\end{array}$ \\
\hline Bush Vote O & $\begin{array}{l}-0.0208 \\
(0.0280)\end{array}$ & $\begin{array}{r}-0.0158 \\
(-0.57)\end{array}$ & $\begin{array}{r}-0.0140 \\
(-0.50)\end{array}$ & $\begin{array}{r}-0.0180 \\
(-0.63)\end{array}$ \\
\hline Bush Vote D & $\begin{array}{l}-0.0911^{* * *} \\
(0.0283)\end{array}$ & $\begin{array}{l}-0.0603^{* *} \\
(-2.14)\end{array}$ & $\begin{array}{c}-0.0470^{*} \\
(-1.65)\end{array}$ & $\begin{array}{r}-0.0443 \\
(-1.50)\end{array}$ \\
\hline Cost of Living $\mathrm{O}$ & $\begin{array}{r}0.0098 \\
(0.0197)\end{array}$ & $\begin{array}{r}0.0193 \\
(1.01)\end{array}$ & $\begin{array}{r}0.0164 \\
(0.87)\end{array}$ & $\begin{array}{r}0.0146 \\
(0.78)\end{array}$ \\
\hline Cost of Living D & $\begin{array}{r}0.0083 \\
(0.0212)\end{array}$ & $\begin{array}{r}0.0029 \\
(0.15)\end{array}$ & $\begin{array}{r}0.0004 \\
(0.02)\end{array}$ & $\begin{array}{r}0.0014 \\
(0.08)\end{array}$ \\
\hline Employment Growth O & $\begin{array}{c}0.0802^{* * *} \\
(0.0338)\end{array}$ & $\begin{array}{l}0.0838^{* *} \\
(2.50)\end{array}$ & $\begin{array}{l}0.0941^{* * *} \\
(2.65)\end{array}$ & $\begin{array}{l}0.0833^{* *} \\
(2.34)\end{array}$ \\
\hline Employment Growth D & $\begin{array}{l}0.1528^{* * *} \\
(0.0343)\end{array}$ & $\begin{array}{l}0.1100^{* * *} \\
(3.33)\end{array}$ & $\begin{array}{l}0.1275^{* * *} \\
(3.88)\end{array}$ & $\begin{array}{l}0.1171^{* * *} \\
(3.33)\end{array}$ \\
\hline Unemployment O & $\begin{array}{r}0.0099 \\
(0.0969)\end{array}$ & $\begin{array}{r}0.0261 \\
(0.28)\end{array}$ & $\begin{array}{c}0.0706^{*} \\
(0.74)\end{array}$ & $\begin{array}{r}0.0648 \\
(0.66)\end{array}$ \\
\hline Unemployment D & $\begin{array}{l}-0.3690^{* * *} \\
(0.1019)\end{array}$ & $\begin{array}{l}-0.2557^{* * *} \\
(-2.77)\end{array}$ & $\begin{array}{c}-0.1823^{*} \\
(-1.95)\end{array}$ & $\begin{array}{c}-0.1834^{*} \\
(-1.90)\end{array}$ \\
\hline Income $\mathrm{O}$ & $\begin{array}{r}-0.00002 \\
(0.00004)\end{array}$ & $\begin{array}{r}-0.00002 \\
(-0.53)\end{array}$ & $\begin{array}{r}-0.00002 \\
(-0.42)\end{array}$ & $\begin{array}{r}-0.00001 \\
(-0.26)\end{array}$ \\
\hline Income D & $\begin{array}{r}0.00001 \\
(0.00004)\end{array}$ & $\begin{array}{r}0.00001 \\
(0.29)\end{array}$ & $\begin{array}{r}0.00001 \\
(0.17)\end{array}$ & $\begin{array}{r}0.00001 \\
(0.27)\end{array}$ \\
\hline Partnership Law O & $\begin{array}{r}-0.5554 \\
(0.5029)\end{array}$ & $\begin{array}{r}-0.3115 \\
(-0.67)\end{array}$ & $\begin{array}{r}-0.0647 \\
(-0.13)\end{array}$ & $\begin{array}{r}-0.2766 \\
(-0.58)\end{array}$ \\
\hline Partnership Law D & $\begin{array}{r}-0.1664 \\
(0.4938)\end{array}$ & $\begin{array}{r}-0.0964 \\
(-0.21)\end{array}$ & $\begin{array}{r}0.5362 \\
(1.07)\end{array}$ & $\begin{array}{r}0.0960 \\
(0.21)\end{array}$ \\
\hline Hate Crime Law O & $\begin{array}{r}0.0922 \\
(0.2411)\end{array}$ & $\begin{array}{r}0.2031 \\
(0.84)\end{array}$ & $\begin{array}{r}0.2789 \\
(1.10)\end{array}$ & $\begin{array}{r}0.2412 \\
(0.99)\end{array}$ \\
\hline Hate Crime Law D & $\begin{array}{l}0.4150^{* *} \\
(0.2476)\end{array}$ & $\begin{array}{c}0.4228^{*} \\
(1.81)\end{array}$ & $\begin{array}{l}0.6821^{* * *} \\
(2.67)\end{array}$ & $\begin{array}{l}0.6435^{* *} \\
(2.55)\end{array}$ \\
\hline Public Law O & $\begin{array}{r}0.1233 \\
(0.2806)\end{array}$ & $\begin{array}{r}0.1123 \\
(0.39)\end{array}$ & $\begin{array}{r}0.0443 \\
(0.16)\end{array}$ & $\begin{array}{r}-0.2003 \\
(-0.71)\end{array}$ \\
\hline Public Law D & $\begin{array}{c}0.6587^{* *} \\
(0.2834)\end{array}$ & $\begin{array}{l}0.5435^{* *} \\
(1.99)\end{array}$ & $\begin{array}{l}0.5121^{* *} \\
(2.06)\end{array}$ & $\begin{array}{r}0.4080 \\
(1.43)\end{array}$ \\
\hline Private Law $\mathrm{O}$ & $\begin{array}{r}-0.2869 \\
(0.3838)\end{array}$ & $\begin{array}{r}-0.5282 \\
(-1.45)\end{array}$ & $\begin{array}{l}-0.7853^{* *} \\
(-2.09)\end{array}$ & $\begin{array}{c}-0.6429^{*} \\
(-1.84)\end{array}$ \\
\hline Private Law D & $\begin{array}{l}-0.9007^{* *} \\
(0.3815)\end{array}$ & $\begin{array}{c}-0.7856^{* *} \\
(-2.15)\end{array}$ & $\begin{array}{c}-1.1790^{* * * *} \\
(-3.25)\end{array}$ & $\begin{array}{l}-0.9896^{* * *} \\
(-2.87)\end{array}$ \\
\hline Rho & & $0.1240^{* * *}$ & $0.1211^{* * *}$ & $0.1211^{* * *}$ \\
\hline
\end{tabular}

${ }^{\text {a }}$ Statistical significance is reported at the $1 \%(* * *), 5 \%(* *)$, and $10 \%(*)$ levels.

b Standard deviations are reported in parenthesis for the the non-spatial Tobit model.

c $\mathrm{T}$-statistics are reported in parenthesis for the spatial Tobit models. 
Table 3.7: Bayesian Spatial Autoregressive Tobit Model Estimation Results for Separate Gay and Lesbian Samples

\begin{tabular}{|c|c|c|c|c|c|c|}
\hline & $\begin{array}{l}\text { Gays } \\
\text { (1) } \\
1994 \text { Cutoff }\end{array}$ & $\begin{array}{c}(2) \\
1995 \text { Cutoff }\end{array}$ & $\begin{array}{c}(3) \\
1996 \text { Cutoff }\end{array}$ & $\begin{array}{c}\text { Lesbians } \\
\text { (4) } \\
1994 \text { Cutoff }\end{array}$ & $\begin{array}{c}(5) \\
1995 \text { Cutoff }\end{array}$ & $\begin{array}{c}(6) \\
1996 \text { Cutoff }\end{array}$ \\
\hline Stayer & $\begin{array}{l}-42.59^{* * *} \\
(-6.58)\end{array}$ & $\begin{array}{l}-47.06^{* * * *} \\
(-6.98)\end{array}$ & $\begin{array}{l}-46.64^{* * *} \\
(-7.15)\end{array}$ & $\begin{array}{l}-24.47^{* * *} \\
(-3.49)\end{array}$ & $\begin{array}{l}-26.54^{* * *} \\
(-4.16)\end{array}$ & $\begin{array}{l}-25.46^{* * *} \\
(-4.11)\end{array}$ \\
\hline Mover & $\begin{array}{l}-48.35^{* * *} \\
(-7.50)\end{array}$ & $\begin{array}{l}-52.75^{* * *} \\
(-7.87)\end{array}$ & $\begin{array}{l}-52.36^{* * *} \\
(-8.04)\end{array}$ & $\begin{array}{l}-29.64^{* * *} \\
(-4.26)\end{array}$ & $\begin{array}{l}-31.66^{* * *} \\
(-4.96)\end{array}$ & $\begin{array}{l}-30.56^{* * *} \\
(-4.97)\end{array}$ \\
\hline Distance & $\begin{array}{l}-0.6754^{* * *} \\
(-10.41)\end{array}$ & $\begin{array}{l}-0.6872^{* * *} \\
(-10.78)\end{array}$ & $\begin{array}{l}-0.6862^{* * *} \\
(-10.98)\end{array}$ & $\begin{array}{l}-0.8537^{* * *} \\
(-14.53)\end{array}$ & $\begin{array}{l}-0.8604^{* * *} \\
(-13.47)\end{array}$ & $\begin{array}{l}-0.8576^{* * *} \\
(-13.31)\end{array}$ \\
\hline Distance Squared & $\begin{array}{l}0.0205^{* * *} \\
(7.67)\end{array}$ & $\begin{array}{l}0.0208^{* * *} \\
(8.04)\end{array}$ & $\begin{array}{l}0.0206^{* * *} \\
(8.26)\end{array}$ & $\begin{array}{l}0.0279^{* * *} \\
(11.50)\end{array}$ & $\begin{array}{l}0.0282^{* * *} \\
(10.88)\end{array}$ & $\begin{array}{l}0.0280^{* * *} \\
(10.72)\end{array}$ \\
\hline Log Population O & $\begin{array}{l}1.78^{* * *} \\
(9.82)\end{array}$ & $\begin{array}{c}1.83^{* * *} \\
(10.71)\end{array}$ & $\begin{array}{l}1.86^{* * * *} \\
(10.23)\end{array}$ & $\begin{array}{l}1.92^{* * * *} \\
(11.17)\end{array}$ & $\begin{array}{l}1.98^{* * *} \\
(11.67)\end{array}$ & $\begin{array}{l}2.02^{* * *} \\
(11.40)\end{array}$ \\
\hline Log Population D & $\begin{array}{l}1.61^{* * *} \\
(9.32)\end{array}$ & $\begin{array}{c}1.82^{* * *} \\
(10.52)\end{array}$ & $\begin{array}{l}1.78^{* * * *} \\
(10.69)\end{array}$ & $\begin{array}{l}1.69^{* * *} \\
(10.23)\end{array}$ & $\begin{array}{l}1.86^{* * *} \\
(11.31)\end{array}$ & $\begin{array}{l}1.84^{* * *} \\
(10.94)\end{array}$ \\
\hline Cooling O & $\begin{array}{l}0.0006^{* *} \\
(1.97)\end{array}$ & $\begin{array}{r}0.0005 \\
(1.62)\end{array}$ & $\begin{array}{c}0.0005^{*} \\
(1.85)\end{array}$ & $\begin{array}{r}-0.0000 \\
(-0.06)\end{array}$ & $\begin{array}{r}-0.0001 \\
(-0.17)\end{array}$ & $\begin{array}{r}-0.0001 \\
(-0.31)\end{array}$ \\
\hline Cooling D & $\begin{array}{r}-0.0000 \\
(-0.09)\end{array}$ & $\begin{array}{r}-0.0002 \\
(-0.66)\end{array}$ & $\begin{array}{r}-0.0002 \\
(-0.69)\end{array}$ & $\begin{array}{l}-0.0005^{* *} \\
(-2.08)\end{array}$ & $\begin{array}{l}-0.0008^{* * *} \\
(-2.77)\end{array}$ & $\begin{array}{l}-0.0007^{* * *} \\
(-2.64)\end{array}$ \\
\hline Heating $\mathrm{O}$ & $\begin{array}{c}0.0003^{*} \\
(1.92)\end{array}$ & $\begin{array}{c}0.0003^{*} \\
(1.81)\end{array}$ & $\begin{array}{l}0.0003^{* *} \\
(2.21)\end{array}$ & $\begin{array}{r}0.0001 \\
(0.54)\end{array}$ & $\begin{array}{r}0.0001 \\
(0.62)\end{array}$ & $\begin{array}{r}0.0001 \\
(0.57)\end{array}$ \\
\hline Heating D & $\begin{array}{r}-0.0001 \\
(-0.95)\end{array}$ & $\begin{array}{r}-0.0001 \\
(-0.85)\end{array}$ & $\begin{array}{r}-0.0001 \\
(-0.84)\end{array}$ & $\begin{array}{l}-0.0004^{* * *} \\
(-2.80)\end{array}$ & $\begin{array}{l}-0.0004^{* * *} \\
(-3.21)\end{array}$ & $\begin{array}{l}-0.0004^{* * *} \\
(-3.24)\end{array}$ \\
\hline Wind $\mathrm{O}$ & $\begin{array}{l}0.2255^{* * *} \\
(3.13)\end{array}$ & $\begin{array}{l}0.2378^{* * *} \\
(3.02)\end{array}$ & $\begin{array}{l}0.2249^{* * *} \\
(2.91)\end{array}$ & $\begin{array}{l}0.2153^{* * *} \\
(2.89)\end{array}$ & $\begin{array}{l}0.2375^{* * *} \\
(2.96)\end{array}$ & $\begin{array}{l}0.2291^{* * *} \\
(2.85)\end{array}$ \\
\hline Wind D & $\begin{array}{r}0.0918 \\
(1.19)\end{array}$ & $\begin{array}{c}0.1402^{*} \\
(1.79)\end{array}$ & $\begin{array}{r}0.1125 \\
(1.53)\end{array}$ & $\begin{array}{r}0.07356 \\
(0.93)\end{array}$ & $\begin{array}{r}0.1169 \\
(1.55)\end{array}$ & $\begin{array}{r}0.0710 \\
(0.96)\end{array}$ \\
\hline Sunshine O & $\begin{array}{r}0.0281 \\
(1.20)\end{array}$ & $\begin{array}{r}0.0337 \\
(1.42)\end{array}$ & $\begin{array}{r}0.0301 \\
(1.22)\end{array}$ & $\begin{array}{l}0.0534^{* *} \\
(2.25)\end{array}$ & $\begin{array}{l}0.0583^{* *} \\
(2.46)\end{array}$ & $\begin{array}{l}0.0530^{* *} \\
(2.21)\end{array}$ \\
\hline Sunshine D & $\begin{array}{c}0.0429^{*} \\
(1.81)\end{array}$ & $\begin{array}{c}0.0583^{* *} \\
(2.54)\end{array}$ & $\begin{array}{c}0.0500^{* *} \\
(2.24)\end{array}$ & $\begin{array}{c}0.0539^{* *} \\
(2.29)\end{array}$ & $\begin{array}{c}0.0599^{* *} \\
(2.56)\end{array}$ & $\begin{array}{c}0.0532^{* *} \\
(2.36)\end{array}$ \\
\hline Humidity $\mathrm{O}$ & $\begin{array}{r}-0.0126 \\
(-0.51)\end{array}$ & $\begin{array}{r}-0.0096 \\
(-0.41)\end{array}$ & $\begin{array}{r}-0.0093 \\
(-0.38)\end{array}$ & $\begin{array}{r}0.0376 \\
(1.52)\end{array}$ & $\begin{array}{c}0.0420^{*} \\
(1.77)\end{array}$ & $\begin{array}{r}0.0369 \\
(1.46)\end{array}$ \\
\hline Humidity D & $\begin{array}{r}-0.0131 \\
(-0.57)\end{array}$ & $\begin{array}{r}-0.0084 \\
(-0.36)\end{array}$ & $\begin{array}{r}-0.0123 \\
(-0.55)\end{array}$ & $\begin{array}{r}-0.0339 \\
(-1.46)\end{array}$ & $\begin{array}{c}-0.0412^{*} \\
(-1.77)\end{array}$ & $\begin{array}{c}-0.0419^{*} \\
(-1.86)\end{array}$ \\
\hline Coastline $\mathrm{O}$ & $\begin{array}{r}-0.2566 \\
(-0.68)\end{array}$ & $\begin{array}{r}-0.3663 \\
(-1.04)\end{array}$ & $\begin{array}{r}-0.3639 \\
(-0.91)\end{array}$ & $\begin{array}{r}0.1804 \\
(0.51)\end{array}$ & $\begin{array}{r}0.1462 \\
(0.41)\end{array}$ & $\begin{array}{r}0.1274 \\
(0.34)\end{array}$ \\
\hline Coastline D & $\begin{array}{r}0.5286 \\
(1.48)\end{array}$ & $\begin{array}{r}0.3606 \\
(1.00)\end{array}$ & $\begin{array}{r}0.2337 \\
(0.64)\end{array}$ & $\begin{array}{c}0.8400^{* *} \\
(2.40)\end{array}$ & $\begin{array}{c}0.6702^{* *} \\
(1.97)\end{array}$ & $\begin{array}{r}0.5026 \\
(1.34)\end{array}$ \\
\hline Water Cover O & $\begin{array}{r}0.0209 \\
(0.41)\end{array}$ & $\begin{array}{r}0.0497 \\
(1.04)\end{array}$ & $\begin{array}{r}0.0414 \\
(0.78)\end{array}$ & $\begin{array}{r}-0.0335 \\
(-0.72)\end{array}$ & $\begin{array}{r}-0.0051 \\
(-0.10)\end{array}$ & $\begin{array}{r}-0.0020 \\
(-0.04)\end{array}$ \\
\hline Water Cover D & $\begin{array}{r}0.0079 \\
(0.17)\end{array}$ & $\begin{array}{r}0.0569 \\
(1.10)\end{array}$ & $\begin{array}{r}0.0664 \\
(1.36)\end{array}$ & $\begin{array}{r}0.0348 \\
(0.76)\end{array}$ & $\begin{array}{c}0.0978^{* *} \\
(2.04)\end{array}$ & $\begin{array}{c}0.0983^{*} \\
(2.07)\end{array}$ \\
\hline State Park O & $\begin{array}{r}-0.1828 \\
(-1.56)\end{array}$ & $\begin{array}{r}-0.1862 \\
(-1.64)\end{array}$ & $\begin{array}{r}-0.1874 \\
(-1.53)\end{array}$ & $\begin{array}{r}0.0373 \\
(0.33)\end{array}$ & $\begin{array}{r}-0.0028 \\
(-0.02)\end{array}$ & $\begin{array}{r}0.0113 \\
(0.10)\end{array}$ \\
\hline State Park D & $\begin{array}{r}-0.1347 \\
(-1.17)\end{array}$ & $\begin{array}{c}-0.1977^{*} \\
(-1.72)\end{array}$ & $\begin{array}{c}-0.2523^{* *} \\
(-2.14)\end{array}$ & $\begin{array}{r}0.1027 \\
(0.85)\end{array}$ & $\begin{array}{r}0.0241 \\
(0.21)\end{array}$ & $\begin{array}{r}-0.0308 \\
(-0.26)\end{array}$ \\
\hline
\end{tabular}

${ }^{\text {a }}$ Statistical significance is reported at the $1 \%(* * *), 5 \%(* *)$, and $10 \%(*)$ levels.

$\mathrm{b}$ T-statistics are reported in parenthesis for the spatial Tobit models.

Continued on next page... 
Table 3.8: (Continued): Bayesian Spatial Autoregressive Tobit Model Estimation Results for Separate Gay and Lesbian Samples

\begin{tabular}{|c|c|c|c|c|c|c|}
\hline & $\begin{array}{l}\text { Gays } \\
\text { (1) } \\
1994 \text { Cutoff }\end{array}$ & $\begin{array}{c}(2) \\
1995 \text { Cutoff }\end{array}$ & $\begin{array}{c}\text { (3) } \\
1996 \text { Cutoff }\end{array}$ & $\begin{array}{c}\text { Lesbians } \\
\text { (4) } \\
1994 \text { Cutoff }\end{array}$ & $\begin{array}{c}(5) \\
1995 \text { Cutoff }\end{array}$ & $\begin{array}{c}(6) \\
1996 \text { Cutoff }\end{array}$ \\
\hline Crime Rate $\mathrm{O}$ & $\begin{array}{r}-0.00005 \\
(-0.47)\end{array}$ & $\begin{array}{r}-0.00007 \\
(-0.60)\end{array}$ & $\begin{array}{r}-0.00003 \\
(-0.28)\end{array}$ & $\begin{array}{r}0.00004 \\
(0.40)\end{array}$ & $\begin{array}{r}0.00000 \\
(-0.00)\end{array}$ & $\begin{array}{r}0.00000 \\
(0.00)\end{array}$ \\
\hline Crime Rate D & $\begin{array}{c}0.00023^{* *} \\
(2.02)\end{array}$ & $\begin{array}{r}0.00015 \\
(1.26)\end{array}$ & $\begin{array}{r}0.00019 \\
(1.60)\end{array}$ & $\begin{array}{r}-0.00002 \\
(-0.13)\end{array}$ & $\begin{array}{r}-0.00007 \\
(-0.66)\end{array}$ & $\begin{array}{r}-0.00004 \\
(-0.39\end{array}$ \\
\hline Bush Vote $\mathrm{O}$ & $\begin{array}{r}0.0075 \\
(0.21)\end{array}$ & $\begin{array}{r}0.0106 \\
(0.27)\end{array}$ & $\begin{array}{r}0.0024 \\
(0.06)\end{array}$ & $\begin{array}{r}-0.0552 \\
(-1.64)\end{array}$ & $\begin{array}{c}-0.0619^{*} \\
(-1.75)\end{array}$ & $\begin{array}{c}-0.0646^{*} \\
(-1.68)\end{array}$ \\
\hline Bush Vote D & $\begin{array}{r}-0.0201 \\
(-0.56)\end{array}$ & $\begin{array}{c}-0.0064 \\
(-0.17)\end{array}$ & $\begin{array}{r}0.0058 \\
(0.15)\end{array}$ & $\begin{array}{l}-0.0923^{* * *} \\
(-2.63)\end{array}$ & $\begin{array}{l}-0.0764^{* *} \\
(-2.16)\end{array}$ & $\begin{array}{c}-0.0702^{*} \\
(-1.88)\end{array}$ \\
\hline Cost of Living $\mathrm{O}$ & $\begin{array}{r}0.0338 \\
(1.43)\end{array}$ & $\begin{array}{r}0.0252 \\
(1.05)\end{array}$ & $\begin{array}{r}0.0249 \\
(0.96)\end{array}$ & $\begin{array}{r}0.0118 \\
(0.47)\end{array}$ & $\begin{array}{r}0.0124 \\
(0.53)\end{array}$ & $\begin{array}{r}0.0100 \\
(0.41)\end{array}$ \\
\hline Cost of Living D & $\begin{array}{r}0.0405 \\
(1.64)\end{array}$ & $\begin{array}{r}0.0366 \\
(1.49)\end{array}$ & $\begin{array}{r}0.03880 \\
(1.57)\end{array}$ & $\begin{array}{r}-0.0214 \\
(-0.84)\end{array}$ & $\begin{array}{r}-0.0353 \\
(-1.53)\end{array}$ & $\begin{array}{r}-0.0340 \\
(-1.47)\end{array}$ \\
\hline Employment Growth O & $\begin{array}{c}0.10070^{* *} \\
(2.33)\end{array}$ & $\begin{array}{l}0.0976^{* *} \\
(2.32)\end{array}$ & $\begin{array}{c}0.0850^{*} \\
(1.75)\end{array}$ & $\begin{array}{l}0.0988^{* *} \\
(2.23)\end{array}$ & $\begin{array}{l}0.1143^{* *} \\
(2.58)\end{array}$ & $\begin{array}{l}0.1080^{* *} \\
(2.46)\end{array}$ \\
\hline Employment Growth D & $\begin{array}{l}0.1673^{* * *} \\
(4.05)\end{array}$ & $\begin{array}{l}0.1871^{* * *} \\
(4.01)\end{array}$ & $\begin{array}{l}0.1746^{* * *} \\
(3.77)\end{array}$ & $\begin{array}{l}0.1066^{* *} \\
(2.40)\end{array}$ & $\begin{array}{l}0.1080^{* *} \\
(2.49)\end{array}$ & $\begin{array}{l}0.0892^{* *} \\
(1.99)\end{array}$ \\
\hline Unemployment $\mathrm{O}$ & $\begin{array}{l}0.3562^{* * *} \\
(2.82)\end{array}$ & $\begin{array}{l}0.3891^{* * *} \\
(3.20)\end{array}$ & $\begin{array}{l}0.3863^{* * *} \\
(3.04)\end{array}$ & $\begin{array}{r}-0.1634 \\
(-1.32)\end{array}$ & $\begin{array}{r}-0.1245 \\
(-0.96)\end{array}$ & $\begin{array}{r}-0.1241 \\
(-1.00)\end{array}$ \\
\hline Unemployment D & $\begin{array}{r}-0.0683 \\
(-0.54)\end{array}$ & $\begin{array}{r}0.0158 \\
(0.13)\end{array}$ & $\begin{array}{r}0.0277 \\
(0.22)\end{array}$ & $\begin{array}{l}-0.3853^{* * *} \\
(-3.03)\end{array}$ & $\begin{array}{l}-0.3278^{* * *} \\
(-2.72)\end{array}$ & $\begin{array}{l}-0.3235^{* *} \\
(-2.51)\end{array}$ \\
\hline Income $\mathrm{O}$ & $\begin{array}{r}0.00001 \\
(0.09)\end{array}$ & $\begin{array}{r}0.00002 \\
(0.27)\end{array}$ & $\begin{array}{r}0.00002 \\
(0.44)\end{array}$ & $\begin{array}{r}0.00000 \\
(0.00)\end{array}$ & $\begin{array}{r}0.00001 \\
(0.12)\end{array}$ & $\begin{array}{r}0.00001 \\
(0.21)\end{array}$ \\
\hline Income D & $\begin{array}{r}0.00003 \\
(0.58)\end{array}$ & $\begin{array}{r}0.00003 \\
(0.50)\end{array}$ & $\begin{array}{r}0.00003 \\
(0.52)\end{array}$ & $\begin{array}{r}0.00000 \\
(0.04)\end{array}$ & $\begin{array}{r}0.00000 \\
(0.07)\end{array}$ & $\begin{array}{r}0.00001 \\
(0.19)\end{array}$ \\
\hline Partnership Law O & $\begin{array}{r}-0.2936 \\
(-0.49)\end{array}$ & $\begin{array}{r}-0.0723 \\
(-0.11)\end{array}$ & $\begin{array}{r}-0.4651 \\
(-0.79)\end{array}$ & $\begin{array}{r}-0.1947 \\
(-0.32)\end{array}$ & $\begin{array}{r}-0.0815 \\
(-0.13)\end{array}$ & $\begin{array}{r}-0.1416 \\
(-0.23)\end{array}$ \\
\hline Partnership Law D & $\begin{array}{r}-0.3368 \\
(-0.53)\end{array}$ & $\begin{array}{r}0.4853 \\
(0.72)\end{array}$ & $\begin{array}{r}-0.0188 \\
(-0.03)\end{array}$ & $\begin{array}{r}0.5219 \\
(0.84)\end{array}$ & $\begin{array}{r}1.0249 \\
(1.62)\end{array}$ & $\begin{array}{r}0.4838 \\
(0.85)\end{array}$ \\
\hline Hate Crime Law O & $\begin{array}{r}0.3462 \\
(1.13)\end{array}$ & $\begin{array}{r}0.4394 \\
(1.28)\end{array}$ & $\begin{array}{r}0.3687 \\
(1.09)\end{array}$ & $\begin{array}{r}0.0181 \\
(0.06)\end{array}$ & $\begin{array}{r}-0.0150 \\
(-0.05)\end{array}$ & $\begin{array}{r}0.0094 \\
(0.03)\end{array}$ \\
\hline Hate Crime Law D & $\begin{array}{r}0.4768 \\
(1.49)\end{array}$ & $\begin{array}{l}0.8658^{* * *} \\
(2.60)\end{array}$ & $\begin{array}{c}0.7928^{* *} \\
(2.35)\end{array}$ & $\begin{array}{c}0.6394^{* *} \\
(2.17)\end{array}$ & $\begin{array}{l}0.9633^{* * *} \\
(3.00)\end{array}$ & $\begin{array}{l}0.9646^{* * * *} \\
(3.01)\end{array}$ \\
\hline Public Law O & $\begin{array}{r}-0.2008 \\
(-0.55)\end{array}$ & $\begin{array}{r}-0.1499 \\
(-0.43)\end{array}$ & $\begin{array}{r}-0.4718 \\
(-1.33)\end{array}$ & $\begin{array}{r}0.2645 \\
(0.70)\end{array}$ & $\begin{array}{r}0.1234 \\
(0.37)\end{array}$ & $\begin{array}{r}-0.1655 \\
(-0.47)\end{array}$ \\
\hline Public Law D & $\begin{array}{c}0.5724^{*} \\
(1.67)\end{array}$ & $\begin{array}{c}0.5475 \\
(1.60)\end{array}$ & $\begin{array}{r}0.5666 \\
(1.52)\end{array}$ & $\begin{array}{r}0.2981 \\
(0.82)\end{array}$ & $\begin{array}{r}0.2656 \\
(0.82)\end{array}$ & $\begin{array}{r}0.1918 \\
(0.55)\end{array}$ \\
\hline Private Law O & $\begin{array}{r}-0.2039 \\
(-0.42)\end{array}$ & $\begin{array}{r}-0.4642 \\
(-1.00)\end{array}$ & $\begin{array}{r}-0.2866 \\
(-0.63)\end{array}$ & $\begin{array}{r}-0.6412 \\
(-1.30)\end{array}$ & $\begin{array}{r}-0.7142 \\
(-1.57)\end{array}$ & $\begin{array}{r}-0.5789 \\
(-1.36)\end{array}$ \\
\hline Private Law D & $\begin{array}{c}-0.9191^{* *} \\
(-2.02)\end{array}$ & $\begin{array}{l}-1.5433^{* * * *} \\
(-3.33)\end{array}$ & $\begin{array}{l}-1.3429 * * * \\
(-3.07)\end{array}$ & $\begin{array}{c}-1.1113^{* *} \\
(-2.27)\end{array}$ & $\begin{array}{l}-1.3282^{* * * *} \\
(-2.90)\end{array}$ & $\begin{array}{l}-1.1754^{* * *} \\
(-2.78)\end{array}$ \\
\hline rho & $0.1010^{* * *}$ & $0.0951^{* * *}$ & $0.0968^{* * *}$ & $0.0651^{* *}$ & $0.0626^{* *}$ & $0.0595^{* *}$ \\
\hline
\end{tabular}

${ }^{\text {a }}$ Statistical significance is reported at the $1 \%(* * *), 5 \%(* *)$, and $10 \%(*)$ levels.

b $\mathrm{T}$-statistics are reported in parenthesis for the spatial Tobit models. 
Table 3.9: Bayesian Spatial Autoregressive Tobit Model Estimation Results Including Local Laws as of 1994

\begin{tabular}{|c|c|c|c|c|}
\hline & $\begin{array}{c}(1) \\
\text { Non-Spatial }\end{array}$ & $\begin{array}{l}(2) \\
\text { Full }\end{array}$ & $\begin{array}{r}(3) \\
\text { Gays }\end{array}$ & $\begin{array}{c}(4) \\
\text { Lesbians }\end{array}$ \\
\hline Stayer & $\begin{array}{l}-32.38^{* * *} \\
(5.60)\end{array}$ & $\begin{array}{l}-31.23^{* * *} \\
(-5.93)\end{array}$ & $\begin{array}{l}-46.91 * * * \\
(-7.05)\end{array}$ & $\begin{array}{l}-27.87 * * * \\
(-4.27)\end{array}$ \\
\hline Mover & $\begin{array}{l}-36.76^{* * *} \\
(5.54)\end{array}$ & $\begin{array}{l}-35.96^{* * *} \\
(-6.88)\end{array}$ & $\begin{array}{l}-52.67^{* * *} \\
(-7.91)\end{array}$ & $\begin{array}{l}-33.02^{* * *} \\
(-5.09)\end{array}$ \\
\hline Distance & $\begin{array}{l}-0.7950^{* * *} \\
(0.0497)\end{array}$ & $\begin{array}{l}-0.6663^{* * *} \\
(-12.82)\end{array}$ & $\begin{array}{l}-0.6856^{* * *} \\
(-10.27)\end{array}$ & $\begin{array}{l}-0.8543^{* * *} \\
(-13.93)\end{array}$ \\
\hline Distance Squared & $\begin{array}{l}0.0249^{* * *} \\
(0.0021)\end{array}$ & $\begin{array}{l}0.0214^{* * *} \\
(10.28)\end{array}$ & $\begin{array}{l}0.0207^{* * *} \\
(7.74)\end{array}$ & $\begin{array}{l}0.0279^{* * *} \\
(11.08)\end{array}$ \\
\hline Log Population O & $\begin{array}{l}2.07^{* * *} \\
(0.1377)\end{array}$ & $\begin{array}{l}1.93^{* * *} \\
(14.09)\end{array}$ & $\begin{array}{l}1.88^{* * * *} \\
(11.28)\end{array}$ & $\begin{array}{l}2.02^{* * *} \\
(11.91)\end{array}$ \\
\hline Log Population D & $\begin{array}{c}1.84^{* * *} \\
(0.1289)\end{array}$ & $\begin{array}{l}1.42^{* * *} \\
(11.10)\end{array}$ & $\begin{array}{l}1.68^{* * *} \\
(9.20)\end{array}$ & $\begin{array}{l}1.75^{\text {*** }} \\
(10.80)\end{array}$ \\
\hline Cooling O & $\begin{array}{r}0.0002 \\
(0.0002)\end{array}$ & $\begin{array}{r}0.0003 \\
(1.20)\end{array}$ & $\begin{array}{l}0.0006^{* *} \\
(2.06)\end{array}$ & $\begin{array}{r}0.0000 \\
(0.11)\end{array}$ \\
\hline Cooling D & $\begin{array}{l}-0.0004^{* *} \\
(0.0002)\end{array}$ & $\begin{array}{r}-0.0003 \\
(-1.29)\end{array}$ & $\begin{array}{r}0.0000 \\
(0.03)\end{array}$ & $\begin{array}{r}-0.0005 \\
(-1.55)\end{array}$ \\
\hline Heating $\mathrm{O}$ & $\begin{array}{l}0.0003^{* *} \\
(0.0001)\end{array}$ & $\begin{array}{l}0.0003^{* * *} \\
(2.70)\end{array}$ & $\begin{array}{l}0.0004^{* *} \\
(2.37)\end{array}$ & $\begin{array}{r}0.0002 \\
(0.97)\end{array}$ \\
\hline Heating D & $\begin{array}{l}-0.0004^{* * *} \\
(0.0001)\end{array}$ & $\begin{array}{l}-0.0003^{* *} \\
(-2.56)\end{array}$ & $\begin{array}{r}-0.0001 \\
(-0.50)\end{array}$ & $\begin{array}{l}-0.0003^{* *} \\
(-2.10)\end{array}$ \\
\hline Wind $\mathrm{O}$ & $\begin{array}{l}0.1708^{* * *} \\
(0.0641)\end{array}$ & $\begin{array}{l}0.1791^{* * *} \\
(3.01)\end{array}$ & $\begin{array}{l}0.2270^{* * *} \\
(2.92)\end{array}$ & $\begin{array}{l}0.2206^{* * *} \\
(2.69)\end{array}$ \\
\hline Wind D & $\begin{array}{r}0.0170 \\
(0.0624)\end{array}$ & $\begin{array}{r}0.0613 \\
(1.07)\end{array}$ & $\begin{array}{r}0.0870 \\
(1.07)\end{array}$ & $\begin{array}{r}0.0560 \\
(0.72)\end{array}$ \\
\hline Sunshine O & $\begin{array}{l}0.0490^{* * *} \\
(0.0198)\end{array}$ & $\begin{array}{l}0.0507^{* * *} \\
(2.81)\end{array}$ & $\begin{array}{r}0.0330 \\
(1.46)\end{array}$ & $\begin{array}{l}0.0529 * * \\
(2.13)\end{array}$ \\
\hline Sunshine D & $\begin{array}{c}0.0438^{* * *} \\
(0.01922)\end{array}$ & $\begin{array}{l}0.0431^{* *} \\
(2.37)\end{array}$ & $\begin{array}{c}0.0461^{*} \\
(1.92)\end{array}$ & $\begin{array}{l}0.0579^{* * *} \\
(2.44)\end{array}$ \\
\hline Humidity $\mathrm{O}$ & $\begin{array}{r}0.0192 \\
(0.0212)\end{array}$ & $\begin{array}{r}0.0185 \\
(0.95)\end{array}$ & $\begin{array}{r}-0.0081 \\
(-0.33)\end{array}$ & $\begin{array}{l}0.0423^{* *} \\
(1.76)\end{array}$ \\
\hline Humidity D & $\begin{array}{r}-0.0204 \\
(0.0194)\end{array}$ & $\begin{array}{r}-0.0160 \\
(-0.89)\end{array}$ & $\begin{array}{r}-0.0080 \\
(-0.31)\end{array}$ & $\begin{array}{r}-0.0287 \\
(-1.19)\end{array}$ \\
\hline Coastline $\mathrm{O}$ & $\begin{array}{r}-0.2862 \\
(0.2925)\end{array}$ & $\begin{array}{r}-0.1631 \\
(-0.59)\end{array}$ & $\begin{array}{r}-0.2679 \\
(-0.79)\end{array}$ & $\begin{array}{r}0.1392 \\
(0.41)\end{array}$ \\
\hline Coastline D & $\begin{array}{l}0.6608^{* * *} \\
(0.2910)\end{array}$ & $\begin{array}{l}0.6228^{* *} \\
(2.40)\end{array}$ & $\begin{array}{r}0.5633 \\
(1.56)\end{array}$ & $\begin{array}{l}0.8416^{* *} \\
(2.41)\end{array}$ \\
\hline Water Cover O & $\begin{array}{r}0.0138 \\
(0.0402)\end{array}$ & $\begin{array}{r}0.0098 \\
(0.25)\end{array}$ & $\begin{array}{r}0.0084 \\
(0.17)\end{array}$ & $\begin{array}{r}-0.0358 \\
(-0.74)\end{array}$ \\
\hline Water Cover D & $\begin{array}{r}0.0103 \\
(0.0377)\end{array}$ & $\begin{array}{r}0.0087 \\
(0.25)\end{array}$ & $\begin{array}{r}-0.0021 \\
(-0.04)\end{array}$ & $\begin{array}{r}0.0299 \\
(0.65)\end{array}$ \\
\hline State Park O & $\begin{array}{r}0.0212 \\
(0.0944)\end{array}$ & $\begin{array}{r}0.0024 \\
(0.02)\end{array}$ & $\begin{array}{r}-0.1430 \\
(-1.21)\end{array}$ & $\begin{array}{r}0.0537 \\
(0.45)\end{array}$ \\
\hline State Park D & $\begin{array}{r}-0.0045 \\
(0.0944)\end{array}$ & $\begin{array}{r}-0.0292 \\
(-0.32)\end{array}$ & $\begin{array}{r}-0.1583 \\
(-1.35)\end{array}$ & $\begin{array}{r}0.1070 \\
(0.87)\end{array}$ \\
\hline
\end{tabular}

${ }^{a}$ Statistical significance is reported at the $1 \%(* * *), 5 \%(* *)$, and $10 \%(*)$ levels.

b Standard deviations are reported in parenthesis for the non-spatial Tobit model.

c T-statistics are reported in parenthesis for the spatial Tobit models.

Continued on next page... 
Table 3.10: (Continued): Bayesian Spatial Autoregressive Tobit Model Estimation Results Including Local Laws as of 1994

\begin{tabular}{|c|c|c|c|c|}
\hline & $\begin{array}{c}(1) \\
\text { Non-Spatial }\end{array}$ & $\begin{array}{l}(2) \\
\text { Full }\end{array}$ & $\begin{array}{r}(3) \\
\text { Gays }\end{array}$ & $\begin{array}{c}(4) \\
\text { Lesbians }\end{array}$ \\
\hline Crime Rate $\mathrm{O}$ & $\begin{array}{r}0.00009 \\
(0.00009)\end{array}$ & $\begin{array}{r}0.00006 \\
(0.73)\end{array}$ & $\begin{array}{r}-0.00002 \\
(-0.13)\end{array}$ & $\begin{array}{r}0.00006 \\
(0.54)\end{array}$ \\
\hline Crime Rate D & $\begin{array}{r}0.00008 \\
(0.00009)\end{array}$ & $\begin{array}{r}0.00009 \\
(1.07)\end{array}$ & $\begin{array}{c}0.00025^{* *} \\
(2.27)\end{array}$ & $\begin{array}{r}0.00001 \\
(0.10)\end{array}$ \\
\hline Bush Vote O & $\begin{array}{l}-0.0140 \\
(0.0277)\end{array}$ & $\begin{array}{r}-0.0111 \\
(-0.42)\end{array}$ & $\begin{array}{r}0.0110 \\
(0.31)\end{array}$ & $\begin{array}{c}-0.0568^{*} \\
(-1.70)\end{array}$ \\
\hline Bush Vote D & $\begin{array}{l}-0.0830^{* * *} \\
(0.0284)\end{array}$ & $\begin{array}{l}-0.0543^{* *} \\
(-2.01)\end{array}$ & $\begin{array}{r}-0.0047 \\
(-0.13)\end{array}$ & $\begin{array}{l}-0.0900^{* * *} \\
(-2.63)\end{array}$ \\
\hline Cost of Living $\mathrm{O}$ & $\begin{array}{r}0.0115 \\
(0.0197)\end{array}$ & $\begin{array}{r}0.0191 \\
(0.99)\end{array}$ & $\begin{array}{r}0.0307 \\
(1.21)\end{array}$ & $\begin{array}{r}0.0155 \\
(0.61)\end{array}$ \\
\hline Cost of Living D & $\begin{array}{r}0.0077 \\
(0.0202)\end{array}$ & $\begin{array}{r}0.0036 \\
(0.19)\end{array}$ & $\begin{array}{r}0.0403 \\
(1.60)\end{array}$ & $\begin{array}{r}-0.0263 \\
(-1.06)\end{array}$ \\
\hline Employment Growth O & $\begin{array}{l}0.0769^{* *} \\
(0.0347)\end{array}$ & $\begin{array}{l}0.0801^{* *} \\
(2.42)\end{array}$ & $\begin{array}{l}0.0942^{* *} \\
(2.15)\end{array}$ & $\begin{array}{l}0.1024^{* *} \\
(2.30)\end{array}$ \\
\hline Employment Growth D & $\begin{array}{r}0.1500 \\
(0.0341)\end{array}$ & $\begin{array}{l}0.1108^{* * *} \\
(3.38)\end{array}$ & $\begin{array}{l}0.1624^{* * *} \\
(3.82)\end{array}$ & $\begin{array}{l}0.0936^{* *} \\
(2.26)\end{array}$ \\
\hline Unemployment $\mathrm{O}$ & $\begin{array}{r}0.0346 \\
(0.0999)\end{array}$ & $\begin{array}{r}0.0437 \\
(0.44)\end{array}$ & $\begin{array}{l}0.3832^{\text {*** }} \\
(3.15)\end{array}$ & $\begin{array}{r}-0.1372 \\
(-1.07)\end{array}$ \\
\hline Unemployment D & $\begin{array}{l}-0.3412^{* *} \\
(0.1044)\end{array}$ & $\begin{array}{l}-0.2392^{* *} \\
(-2.51)\end{array}$ & $\begin{array}{r}-0.0569 \\
(-0.45)\end{array}$ & $\begin{array}{l}-0.3934^{* * *} \\
(-3.15)\end{array}$ \\
\hline Income $\mathrm{O}$ & $\begin{array}{r}-0.00002 \\
(0.00004)\end{array}$ & $\begin{array}{r}-0.00002 \\
(-0.55)\end{array}$ & $\begin{array}{r}0.00001 \\
(0.18)\end{array}$ & $\begin{array}{r}0.00000 \\
(0.00)\end{array}$ \\
\hline Income D & $\begin{array}{r}0.00001 \\
(0.00004)\end{array}$ & $\begin{array}{r}0.00001 \\
(0.23)\end{array}$ & $\begin{array}{r}0.00003 \\
(0.56)\end{array}$ & $\begin{array}{r}0.00001 \\
(0.13)\end{array}$ \\
\hline Partnership Law O & $\begin{array}{r}-0.0025 \\
(0.0050)\end{array}$ & $\begin{array}{r}0.0005 \\
(0.10)\end{array}$ & $\begin{array}{r}0.0013 \\
(0.21)\end{array}$ & $\begin{array}{r}-0.0011 \\
(-0.17)\end{array}$ \\
\hline Partnership Law D & $\begin{array}{r}0.0032 \\
(0.0051)\end{array}$ & $\begin{array}{r}0.0027 \\
(0.58)\end{array}$ & $\begin{array}{r}0.0021 \\
(0.34)\end{array}$ & $\begin{array}{r}0.0082 \\
(1.35)\end{array}$ \\
\hline Hate Crime Law O & $\begin{array}{r}0.1855 \\
(0.2329)\end{array}$ & $\begin{array}{r}0.2876 \\
(1.21)\end{array}$ & $\begin{array}{c}0.4852^{*} \\
(1.66)\end{array}$ & $\begin{array}{r}0.0360 \\
(0.13)\end{array}$ \\
\hline Hate Crime Law D & $\begin{array}{l}0.4821 \text { ** } \\
(0.2420)\end{array}$ & $\begin{array}{l}0.4566^{* *} \\
(2.08)\end{array}$ & $\begin{array}{c}0.5614^{*} \\
(1.85)\end{array}$ & $\begin{array}{l}0.6741^{* * *} \\
(2.36)\end{array}$ \\
\hline Public Law O & $\begin{array}{r}0.0002 \\
(0.0035)\end{array}$ & $\begin{array}{r}0.0000 \\
(0.01)\end{array}$ & $\begin{array}{r}-0.0029 \\
(-0.63)\end{array}$ & $\begin{array}{r}0.0017 \\
(0.38)\end{array}$ \\
\hline Public Law D & $\begin{array}{l}0.0078^{* *} \\
(0.0036)\end{array}$ & $\begin{array}{c}0.0064^{*} \\
(1.93)\end{array}$ & $\begin{array}{c}0.0074^{*} \\
(1.68)\end{array}$ & $\begin{array}{r}0.0030 \\
(0.68)\end{array}$ \\
\hline Private Law $\mathrm{O}$ & $\begin{array}{l}-0.0066^{*} \\
(0.0047)\end{array}$ & $\begin{array}{c}-0.0087^{*} \\
(-1.94)\end{array}$ & $\begin{array}{r}-0.0067 \\
(-1.12)\end{array}$ & $\begin{array}{r}-0.0093 \\
(-1.62)\end{array}$ \\
\hline Private Law D & $\begin{array}{l}-0.0118^{* * *} \\
(0.0050)\end{array}$ & $\begin{array}{l}-0.0101^{* *} \\
(-2.27)\end{array}$ & $\begin{array}{l}-0.0115^{* *} \\
(-2.02)\end{array}$ & $\begin{array}{c}-0.0133^{* *} \\
(-2.32)\end{array}$ \\
\hline rho & & $0.1232^{* * *}$ & $0.0984^{* * *}$ & $0.0659 * *$ \\
\hline
\end{tabular}

a Statistical significance is reported at the $1 \%(* * *), 5 \%(* *)$, and $10 \%\left({ }^{*}\right)$ levels.

b Standard deviations are reported in parenthesis for the non-spatial Tobit model.

${ }^{\mathrm{c}}$ T-statistics are reported in parenthesis for the spatial Tobit models. 


\section{Chapter 4}

\section{An Examination of Prejudice Against Gay and Lesbian Households: A Spatial Hedonic Approach}

\section{Introduction}

The passage of different types of gay and lesbian rights legislation at the local, state, and federal levels of government has been a topic of heated debate in recent years. The argument that is often made against the passage of legislation that prohibits employment and other types of market discrimination against gays and lesbians is that gays and lesbians are not discriminated against by society. Thus, the passage of anti-discrimination legislation only serves the function of extending special privileges to them. Additionally, the case that is often made against allowing gays and lesbians to marry is that by providing this right to them the institution of marriage is ruined. By ruining this institution and thereby causing a breakdown in the traditional family unit, allowing gays and lesbians to marry leads to increases in divorce, abortion rates, the proportion of children born to single women, and the percent of children in female headed households in society. 
Research examining these claims often made against the passage of legislation provides evidence that these claims are likely not to be true. Evidence pointing towards the existence of discrimination is provided by Herek (2009), which shows using survey data that gays and lesbians at least perceive themselves as being targets of hate crimes. Also, Tebaldi and Elmslie (2006) and Allegretto and Arthur (2001) provide evidence that gays face labor market discrimination, and results from Badgett (1995) indicate that both gays and lesbians are subject to labor market discrimination. Additionally, (Langbein and Yost Jr. 2009) finds no statistically significant adverse effect on society in states that allow gays and lesbians to marry.

Thus, the possibility exists that claims made against passing gay and lesbian rights legislation are made based purely on prejudice, or negative attitudes, that some parts of society have towards gays and lesbians. The need exists to determine if prejudice is indeed the driving force behind arguments made against the passage of legislation, since one would think that arguments based solely on prejudice should not hold any weight in the debate regarding the passage of legislation. The only evidence that exists to date on the existence of prejudice in society is from survey data asking individuals their opinions on homosexuality. However, the examination of results from surveys likely does not provide an accurate picture regarding prejudice because it is not clear how accurately people respond to survey questions regarding prejudices they may have.

Another way to examine the existence of prejudice against gays and lesbians is to use a revealed preferences approach. This approach can be performed by analyzing the effect that the presence of gay and lesbian households in a neighborhood have on house prices in that neighborhood. If evidence is found that a higher percentage of gay and lesbian households in a neighborhood causes a reduction in house prices in that neighborhood, then this can be taken as evidence of society revealing their preference not to live around gays and lesbians. This method for analyzing prejudice has been used in a number of previous studies examining the existence of prejudice against blacks and other racial groups in society (Harris, 1999). 
In the debate over gay and lesbian rights legislation, generally those individuals that are conservative are the ones most adamant in their opposition to the passage of legislation. Thus, the existence and the degree of prejudice that exists against gays and lesbians is likely to vary by neighborhood, depending on the social characteristics of that neighborhood. The expectation, therefore, is that gay and lesbian households are likelier to have a negative impact on house prices in a conservative neighborhood than in a liberal one. The possibility also exists that gay and lesbian households may be an amenity to a neighborhood, especially in one that is liberal, thereby increasing house prices in that neighborhood. Gay and lesbian households may be an amenity to a neighborhood as evidence exists that some individuals in society prefer to live in neighborhoods with more diversity and cultural capital, which gay and lesbian households likely contribute to (Bell and Binnie, 2004).

This paper estimates a hedonic price model that incorporates the percentage of households in a neighborhood that are gay or lesbian and an interaction between this variable and a dummy representing if a neighborhood is conservative. This model is estimated at the census tract level for the Columbus, OH MSA using the spatial autoregressive model. The results from this analysis indicate that in liberal neighborhoods a $.1 \%$ point increase in the percentage of gay and lesbian households increases house prices by .81\%, but in conservative neighborhoods it decreases house prices by $.24 \%$. These results are robust to extension of the analysis to the Cleveland, OH MSA, and are consistent with the theory that conservative individuals may hold a prejudice against gays and lesbians.

\section{Literature Review}

The use of the hedonic price model to value goods that are not explicitly traded in markets is first developed by Rosen (1974). As applied to housing, the hedonic price model suggests that the price of a house represents the sum of expenditures on a number of bundled housing characteristics. These housing characteristics not only include tangible characteristics such 
as the size of the house or the number of bedrooms in the house, but also less tangible neighborhood characteristics such as the quality of the school district or the amount of pollution where the house is located. The fact that these less tangible neighborhood characteristics are also capitalized into house prices is formally modeled by Roback (1982).

The theory that a hedonic price model can be used to examine prejudice against a group is based on the model developed by Yinger (1976), which revises the Bailey (1966) border model of segregation. The Yinger model is developed in regards to whites and blacks, but is applicable to the examination of prejudice against gays and lesbians. In the Yinger model, whites and blacks both receive utility or disutility from living near the other race. This model departs from the typical border model in that it allows for whites and blacks to care about the racial composition of where they live. Thus, the model allows for the possibility of prejudice to be examined. Yinger shows that the coefficient on racial composition in a regression on value or rent measures the effect of prejudice in this model. In particular, a negative coefficient on a variable for the percent of neighborhood occupants that is black means that as this percent increases house prices decrease in the neighborhood, strongly suggesting the existence of prejudice. Also, Yinger discusses the possibility that the degree of prejudice, and therefore the magnitude of the coefficient on the racial composition variable, could vary by neighborhood type. Therefore, interactions for neighborhood type should be included into the regression analysis also.

A number of studies have been performed using the hedonic price model to examine the existence of racial prejudice and discrimination. Kiel and Zabel (1996), using data from the American Housing Survey for Denver, Philadelphia, and Chicago, provides evidence that prejudice against blacks exists in all three of these cities. They also show that this prejudice has been increasing overtime in Denver and Philadelphia, while decreasing in Chicago. Myers (2004) examines discrimination and prejudice also using data from the American Housing Survey, but uses variables that better control for neighborhood effects. She estimates a hedonic price model that controls for the race of the household, percent of a neighborhood's 
population that is black, and interactions between the percentage of a neighborhood that is black and how racially integrated that neighborhood is. Her main findings are that black owners pay premiums of around $10 \%$ for housing, indicating the existence of housing market discrimination against blacks. Furthermore, she finds that house values decline in neighborhoods as the percentage of blacks in that neighborhood increase, suggesting the existence of prejudice against blacks.

The hedonic price model that is developed in this paper is similar to previous models, with the exception that it takes into consideration the possibility of housing prices exhibiting spatial dependence. Only recently have hedonic price models begun to incorporate spatial econometrics into the estimation of the models. Brasington and Hite (2005) uses a spatial model to analyze the influence that the distance from a census block group to the nearest pollution site has on the house prices of that census block group. Additionally, Leguizamon (2010) uses a spatial model of individual housing observations to analyze the effect of the size of neighboring houses on the price of a house.

\section{$3 \quad$ Data and Methodology}

The focus of this paper is on housing transactions that occurred in the Columbus, OH MSA in the year 2000. The reason for performing the analysis at the MSA level is that previous studies have suggested the MSA can be considered a segmented housing market. Palmquist (1984) suggests that no segmentation exists within an MSA, but it does exist between MSAs due to moving costs. Brasington and Hite (2005) makes the case that moving costs are not a valid reason for this difference in segmentation because it is likely to be just as costly to move out to a suburban area within an MSA as it is to move to another MSA. Instead, they suggest that the assumption of market segmentation between MSAs is valid because of differing construction costs and job availability. Their reasoning is that it is more difficult and costly to find a job in a new metro area and move to that area than it is to move or find 
a job in the same metro area.

The data on housing used in this study is from that used in Brasington and Haurin (2006), Brasington (2007), and Brasington and Hite (2006). The data set is composed of 20,027 observations of of real estate transactions in the year 2000 for the Columbus, $\mathrm{OH}$ MSA. Included in each observation of a housing market transaction are detailed information on the characteristics of the house being sold and on the neighborhood that the house is located in. This data set additionally includes data on housing transactions for other MSAs located in Ohio. The Columbus MSA is used for the focus of this paper because it does not extend into any other state, therefore full information is available from this data set on it. It is also used because it is not contiguous to any other MSA, making it a clearly segmented housing market. As for the other two large MSAs in Ohio, Cincinnati and Cleveland, the Cincinnati MSA includes part of Kentucky and Indiana and the Cleveland MSA is contiguous to the Akron MSA.

The unit of observation within the Columbus MSA that is used is the census tract. Each census tract observation can be thought of as representing an average or representative house from the census tract. This representative house observation is created by taking the mean of the price and the other characteristics of the houses that were sold in the census tract. For the housing characteristics in which taking the mean does not make sense, proportions are used. The census tract is used as the unit of observation as opposed to individual houses or a different geography level because the census tract is the smallest geography level in which the number of gay and lesbian households is available from the Census.

The neighborhood characteristic's impact on house prices that is of interest in this paper is the percentage of households in a neighborhood, defined here at the census tract level, that are gay or lesbian. The data used to calculate the percentage of gay and lesbian households in each census tract is from the summary files of the 2000 Census. The Census does not ask individuals directly about their sexual orientation, but beginning with the 1990 
Census it has been possible to identify unmarried gay and lesbian partnered households ${ }^{11}$ For the Columbus MSA, there are a total of 2075 gay households and 2181 lesbian households identified on the 2000 Census. Thus, approximately one percent of the households in the Columbus MSA are gay or lesbian households.

One issue that arises with using Census data on same-sex cohabitating couples is whether or not these couples are indeed gay and lesbian. Black et al. (2000) and Carpenter (2004) both give an in depth analysis of this issue with both providing evidence that the same-sex cohabitating couples identified on the Census are likely to be gay and lesbian. Black et al. (2000) finds that the residence of $60 \%$ of partnered gays identified on the 1990 Census are concentrated in just 20 cities. This finding wouldn't be expected if those individuals identified as partnered gays were actually a part of the general non-gay population. They also find that there is a strong correlation between the concentration of partnered gays in cities and the concentration of AIDS deaths among young men in those cities. Carpenter (2004) identifies same-sex cohabitating couples from the Center for Disease Control's Behavioral Risk Factor Surveillance System (BRFSS) and shows that these couples are likely to be gay and lesbian using information on the sexual behavior and family planning of these couples. This finding provides evidence that in general couples that identify themselves as being same-sex couples on surveys are indeed gay or lesbian.

Another issue that arises is the possibility that some same-sex cohabitating couples fail to report themselves as such due possibly to a distrust of government run surveys. This possibility could make the results of this analysis difficult to interpret if those gay and lesbian couples that identify themselves as same-sex couples on the Census are different in someway from those that do not. Black et al. (2000) shows using data from the General Social Survey (GSS) and the National Health and Social Life Survey (NHSS), which are both nongovernment run surveys, that in fact the 1990 Census only identifies $35 \%$ of the total number

\footnotetext{
${ }^{1}$ Gay and lesbian households are identified from the "relationship to household head" heading on the Census. The categories under this heading are spouse, child, inlaw, unmarried partner, and other nonrelative.
} 
of partnered gay couples and $29 \%$ of the total number of partnered lesbian couples. They discuss though that empirical findings for characteristics such as veteran status, education, and earnings for individuals identified as being in same-sex cohabitating couples on the Census are very similar to those for the same-sex cohabitating couples identified in the GSS and NHSS. Also, Carpenter (2004) estimates gay and lesbian wage regressions using same-sex couples from the BRFSS and finds that the results for these wage regressions do not differ from the results of similar wage regressions using the same-sex couples that are identified on the Census.

In order to obtain a complete analysis of the effect gay and lesbian households have on neighborhood house prices, whether or not a neighborhood is conservative needs to be interacted with the percentage of households that are gay or lesbian. At the census tract level, there is not an obvious way to control for if a neighborhood is conservative. The method that is chosen is to create a variable representing whether the census tract is located in a county that George Bush won in the 2000 Presidential Election. There are eight counties that are located in the Columbus MSA, with over $60 \%$ of the vote going to George Bush in seven of them. These counties are coded as being conservative, while the eighth county is coded as being liberal, with $48 \%$ of the vote going to George Bush.

The data for the rest of the variables included in the analysis are from either the Brasington data set or the summary files of the 2000 Census. The representative census tract housing characteristics that are included are age (in quadratic form), house size (in quadratic form), lot size (in quadratic form), partial bathrooms, full bathrooms, proportion of houses with air conditioning, proportion of houses that are one story, proportion of houses with a deck, and proportion of houses with a garage. In order to control for neighborhood characteristics, the proportion of households below the poverty line, proportion of individuals with a graduate degree, median household income, and proportion of individuals that are black are taken from the Census for each census tract. To further control for neighborhood characteristics, the 9 th grade proficiency rate and mills tax rate for the school district that each house in 
the census tract is located in are averaged and included in the regression analysis. Lastly, in order to control for access to amenities, the distance from the center of each census tract to the Columbus CBD is calculated from the Census Tiger Ohio census tract shapefile.

The typical hedonic price model regresses the price of a house on the characteristics of that house and the neighborhood the house is located in using OLS. However, the coefficients from the OLS estimation of the hedonic price model are likely to be biased. The reason for this bias is that the prices of houses located near each other are likely dependent on each other because people should be willing to pay more for a house located in proximity to houses of higher value. This spatial dependence in housing prices necessitates the inclusion of the average of the prices of proximate houses as an independent variable. Thus, to control for this spatial dependence, the house price dependent variable vector is multiplied by a weight matrix representing the spatial relationship in prices. The weight matrix is typically constructed as an $n \times n$ standardized matrix representing a nearest neighbor or contiguity relationship between observations. Since the observations in this analysis are for a geographical unit, the census tract, the weight matrix is constructed as a contiguity matrix. This matrix is constructed by placing a one in the matrix if two census tracts share a border with each other and then dividing across each row by the number of non-zero elements in that row.

This model is known as the spatial autoregressive hedonic price model and is the one used here to estimate the impact of gay and lesbian households on neighborhood house prices. The model takes the form:

$$
v_{i}=\alpha+\rho W v_{i}+\beta_{d} D_{i}+\beta_{h} X_{h i}+\beta_{n} X_{n i}+\epsilon_{i}, \text { where } \epsilon \text { is } N\left(0, \sigma^{2} I_{i}\right)
$$

in which subscript $i$ indicates the census tract, $v$ represents the logged mean census tract house price, $X_{h}$ is a matrix of the mean or proportion of housing characteristics in the census tract, $X_{n}$ is a matrix of neighborhood characteristics of the census tract, $D$ is the distance 
from the center of the census tract to the Columbus CBD, and $W$ is an $n \times n$ spatial weight matrix representing the contiguity relationship between the census tracts within the MSA 2 Included in $X_{n}$, is the variable for the percentage of gay and lesbian households in the census tract, and a variable representing the interaction between this variable and a dummy equaling one if the census tract is in a county George Bush won in 2000.

Previous literature applying spatial econometrics to hedonic price models has used the spatial Durbin model, as opposed to the spatial autoregressive model. The spatial Durbin model includes not only a spatial lag of the dependent variable, but also spatial lags of the independent variables of the model. The choice to use the spatial autoregressive model instead of the spatial Durbin model is made because the housing observations used here are being averaged at the census tract level. This larger size of the geographical unit makes it less likely that a change in the independent variable of an observation has a direct impact on the dependent variable of another observation. Using Bayesian estimation of the spatial autoregressive and spatial Durbin models, it is possible to calculate model probabilities for the true model. Performing the estimation of these two models and calculating the model probabilities indicates that the spatial autoregressive model is indeed the true model.

The use of a spatial model allows direct, indirect, and total effects for each of the independent variables to be calculated. The direct effect represents the average effect that a change in the independent variable of an observation has on it's own dependent variable. This coefficient includes the initial impact of the change in an independent variable on it's dependent variable as well as feedback in the system. This feedback occurs when the change in the dependent variable causes changes in the other dependent variables of the system through the spatial weight matrix that in turn feedback onto the initial dependent variable. The indirect effect represents the average spatial spill over effect that a change in an independent variable has on all other dependent variables, excluding it's own. The total effect

\footnotetext{
${ }^{2}$ For the creation of the weight matrix $\mathrm{W}$, the Ohio census tract shapefile is obtained from the Census Tiger website and centroid $\mathrm{x}, \mathrm{y}$ coordinates for each census tract within the MSA are calculated using GeoDa. These centroid coordinates are then used to calculate the contiguity weight matrix $W$ in Matlab using LeSage's Spatial Econometrics Toolbox.
} 
is the sum of the direct and indirect effects. The results for the direct effects are the main focus of this paper due to the interest in prejudice against gays and lesbians.

\section{Results}

The results for the estimation of the spatial autoregressive hedonic price model for the Columbus MSA are provided in column 1 of table 4.3. The estimate for the coefficient on the spatial weight matrix $\rho$ is positive and statistically significant. This result provides evidence that observations on house prices are spatially dependent. Thus, a model controlling for spatial dependence is superior to one that does not.

Another coefficient of particular interest is the one on the distance to the CBD variable. Gays and lesbians are usually thought of as locating in high amenity areas, so it is necessary to adequately control for access to amenities in the analysis. This coefficient is negative and statistically significant, as is expected if the distance to CBD controls for access to amenities or jobs located in the CBD. The magnitude of this coefficient indicates that a one mile increase in the distance from the CBD reduces house prices by $.37 \%$.

The results for the coefficients on the rest of the housing and neighborhood characteristics are generally as expected. An increase in the number of partial and full bathrooms, size of the house, and lot size of the house leads to an increase in the price of the house, as does a decrease in the age of the house. Additionally, having a deck, air conditioning, and being one story result in an increase in the price of the house. As for the neighborhood characteristics, an increase in median family income and the proportion of individuals with a graduate degree increases the price of the house, while an increase in the percentage of individuals that are black decreases the price of the house.

The estimated coefficient on the percentage of gay and lesbian households variable, and on the interaction term between this variable and whether a census tract is conservative or not, provide evidence that gay and lesbian households may serve as an amenity in liberal neigh- 
borhoods, but prejudice may exist against these households in conservative neighborhoods. In particular, a .1\% point increase in the percentage of households in a liberal neighborhood that are gay or lesbian leads to a $.81 \%$ increase in the price of a house. On the other hand, a $.1 \%$ increase in the percentage of households in a conservative neighborhood that are gay or lesbian results in a $.24 \%$ decrease in the price of a house. These results confirm the prediction of the Yinger model that the coefficient representing prejudice is dependent on the make up of the neighborhood under consideration.

The focus up to this point has been on the Columbus MSA. As a robustness check of the Columbus results, the same model is run for the Cleveland MSA. This MSA is contiguous to the Akron MSA, so it is not clear if it can be considered a segmented housing market. However, results from this re-estimation can be useful for comparison to the Columbus MSA results. In Cleveland there are five counties, with three of these counties won by George Bush in the 2000 Presidential Election. Thus, these three counties are coded as being conservative for the interaction between percentage of gay and lesbian households and whether a neighborhood is conservative.

The results from this re-estimation for the Cleveland sample are provided in column 2 of table 4.3. The coefficient on the percentage of gay and lesbian households variable is positive and statistically significant, as in the Columbus sample. This coefficient indicates that a $.1 \%$ point increase in the percentage of gay and lesbian households increases the price of a house by $.44 \%$. The results also show that the interaction term between the percentage of households that are gay or lesbian and the conservative variable is positive and statistically insignificant for the Cleveland MSA. This result suggests that prejudice does not exist among conservative neighborhoods in the Cleveland MSA like it does in the Columbus MSA. An explanation for this difference may be that the culture significantly differs between these two MSAs. For instance, those that vote for conservative candidates may be more socially conservative in Columbus than in Cleveland.

Another explanation has to do with the distribution of conservatives in the Cleveland 
MSA. In the Columbus MSA, there are four counties that all voted over $60 \%$ for George Bush, with one county voting under $50 \%$. Therefore, a single dummy variable is appropriate for controlling for conservativeness in Columbus. On the other hand, in Cleveland, the vote for George Bush is much more spread out. He received $34 \%$ of the vote in Cuyahoga County, $43 \%$ in Lorain County, $50 \%$ in Lake County, $56 \%$ in Medina County, and $60 \%$ in Geauga County. Due to this variance in the vote, a single dummy variable may not be appropriate for controlling for conservative neighborhoods in the Cleveland MSA. A better approach may be to include a dummy variable and interaction term for each county, and comparing the estimated interaction terms to the Bush vote of each county.

This re-estimation is performed and the results are reported in column 3 of table 4.3 . In this re-estimation the Cuyahoga County dummy term is omitted, meaning that the observed interaction coefficients are relative to the most liberal county in the MSA. The coefficient for the percentage of gay and lesbian households variable indicates that a one percentage point increase in the percentage of gay and lesbian households leads to a statistically significant $.41 \%$ increase in the price of a house in Cuyahoga County. Turning to the county and percentage of households that are gay and lesbian interaction terms, the only one that is statistically significant is the Geauga County interaction. This coefficient indicates that a one percentage point increase in the percentage of gay and lesbian households reduces the price of a house in Geauga County by $2.79 \%$, providing evidence consistent with the theory that discrimination against gays and lesbians does exist in this county.

The results from the Cleveland regression suggest that there is a threshold, at least in relation to votes for conservative candidates, for prejudice to show up in house prices. This threshold appears to be around $60 \%$, as prejudice did not show up in the results for Medina County, in which George Bush received $56 \%$ of the vote. This result matches with the results from the Columbus MSA in which all the neighborhoods labeled as conservative, and in which evidence of prejudice is found, had over $60 \%$ of the vote go to George Bush. The likely reason for this threshold is that counties with less than this amount of conservative 
vote may not have truly socially conservative attitudes, as not everyone that voted for George Bush holds these attitudes.

The negative impact that gays and lesbians have on house prices in conservative Cleveland neighborhoods appears to be much larger than it is in conservative Columbus neighborhoods. The likely reason for this result is the difference in degree of liberalness of the liberal neighborhoods in the two MSAs. In Cleveland, the Geauga County coefficient is relative to the omitted Cuyahoga County variable, and Cuyahoga County is strongly liberal as only $34 \%$ of the 2000 vote went to George Bush. On the other hand, the coefficient for the conservative counties of Columbus are relative to Franklin County, in which George Bush still received $48 \%$ of the vote.

A possible issue with the results that have been reported up to this point is that the percentage of households in a census tract that are gay or lesbian may not be exogenous. If prejudice exists against gays and lesbians in conservative neighborhoods, then there is the chance that gays and lesbians are discriminated against in the housing market in those neighborhoods. The presence of discrimination then leads to less gays and lesbians locating in that neighborhood than the amount that otherwise would. In order to analyze the possibility that discrimination exists, data is needed on the sexual orientation of the household that is purchasing the house. Unfortunately, characteristics of the individuals purchasing the houses is unavailable in the housing data set used in this paper. If discrimination does exist in conservative and not liberal neighborhoods, then this discrimination would bias the coefficients reported here in the direction of not finding prejudice in conservative neighborhoods. Since the results reported for the Columbus and Cleveland MSAs indicate that prejudice does exist in conservative neighborhoods, the existence of discrimination would only strengthen these results. 


\section{Conclusion}

This chapter builds on studies from the hedonic price model literature on prejudice against blacks to analyze the existence of prejudice against gays and lesbians. The results indicate that in the Columbus MSA the price of a house increases in liberal neighborhoods by $.81 \%$ and falls in conservative neighborhoods by $.24 \%$ in response to a $.1 \%$ point increase in the percentage of gay and lesbian households in that neighborhood. For the Cleveland MSA, the results show that a $.1 \%$ point increase in the percentage of gay and lesbian households increases house prices by $.41 \%$ in all neighborhoods except the most conservative ones, in which house prices fall by $2.79 \%$. These results are taken as being consistent with the theory that gay and lesbian households have an amenity effect in liberal neighborhoods and a disamenity effect, likely due to prejudice, in conservative neighborhoods.

These results have important policy implications related to the passage of gay and lesbian rights legislation. If conservative individuals are indeed prejudice against gays and lesbians, then this prejudice is likely to be playing a role in their voting on gay and lesbian rights issues. This is a problem since it seems unfair to leave such issues up to a popular vote when prejudice is having an effect on the outcome of the vote. Also, the possible existence of prejudice indicates that politicians and gay and lesbian rights groups may want to devote more resources in attempting to reduce this prejudice in society. 
Table 4.1: Definition and Source of Variables

\begin{tabular}{|c|c|c|}
\hline Variable & Definition & Source \\
\hline CBD Distance & $\begin{array}{l}\text { distance from center } \\
\text { of census tract to CBD (in miles) }\end{array}$ & Author's Calculation \\
\hline Age & $\begin{array}{l}\text { mean age of houses within } \\
\text { census tract (in hundreds years) }\end{array}$ & Brasington Data Set \\
\hline Lot Size & $\begin{array}{l}\text { mean lot size of houses within } \\
\text { census tract (in ten thousands of square feet) }\end{array}$ & Brasington Data Set \\
\hline House Size & $\begin{array}{l}\text { mean house size of houses within } \\
\text { census tract (in thousands of square feet) }\end{array}$ & Brasington Data Set \\
\hline Air Conditioning & $\begin{array}{l}\text { proportion of houses within } \\
\text { census tract with central air conditioning }\end{array}$ & Brasington Data Set \\
\hline Deck & $\begin{array}{l}\text { proportion of houses within } \\
\text { census tract with a deck }\end{array}$ & Brasington Data Set \\
\hline Garage & $\begin{array}{l}\text { proportion of houses within } \\
\text { census tract with a garage }\end{array}$ & Brasington Data Set \\
\hline One Story & $\begin{array}{l}\text { proportion of houses within } \\
\text { census tract that are one story }\end{array}$ & Brasington Data Set \\
\hline Full Baths & $\begin{array}{l}\text { mean number of full baths for } \\
\text { houses in the census tract }\end{array}$ & Brasington Data Set \\
\hline Partial Baths & $\begin{array}{l}\text { mean number of partial baths for } \\
\text { houses in the census tract }\end{array}$ & Brasington Data Set \\
\hline Tax Rate & $\begin{array}{l}\text { mean tax year } 2000 \text { class } 1 \text { tax rate } \\
\text { faced by houses within census tract }\end{array}$ & Brasington Data Set \\
\hline School Quality & $\begin{array}{l}\text { mean pass rate on } 9 \text { th grade prof. test } \\
\text { faced by houses within census tract }\end{array}$ & Brasington Data Set \\
\hline Income & $\begin{array}{l}\text { mean income per person in the } \\
\text { census tract }\end{array}$ & 2000 Census Summary File \\
\hline Grad Degree & $\begin{array}{l}\text { proportion of census tract population } \\
\text { that has a graduate degree }\end{array}$ & 2000 Census Summary File \\
\hline Poverty & $\begin{array}{l}\text { proportion of households in census } \\
\text { tract that fall below the poverty line }\end{array}$ & 2000 Census Summary File \\
\hline Black & $\begin{array}{l}\text { proportion of census tract population } \\
\text { that is black }\end{array}$ & 2000 Census Summary File \\
\hline Gay/Lesbian & $\begin{array}{l}\text { proportion of households in census } \\
\text { tract that are gay or lesbian }\end{array}$ & 2000 Census Summary File \\
\hline
\end{tabular}


Table 4.2: Descriptive Statistics

\begin{tabular}{lrrrr}
\hline & Columbus & & Cleveland & \\
& Mean & Std. Dev. & Mean & Std. Dev. \\
\hline Log Price & 11.716 & 0.444 & 11.661 & 0.494 \\
One Story & 0.398 & 0.274 & 0.257 & 0.269 \\
Garage & 0.591 & 0.352 & 0.743 & 0.346 \\
Air Conditioner & 0.521 & 0.387 & 0.178 & 0.247 \\
Deck & 0.040 & 0.099 & 0.134 & 0.168 \\
Full Bath & 1.498 & 0.360 & 1.332 & 0.391 \\
Partial Bath & 0.491 & 0.269 & 0.402 & 0.318 \\
Age & 0.429 & 0.279 & 0.566 & 0.262 \\
House Size & 1.664 & 0.391 & 1.670 & 0.478 \\
Lot Size & 2.148 & 3.037 & 2.263 & 4.160 \\
School Quality & 58.512 & 19.267 & 54.230 & 25.266 \\
Tax Rate & 32.338 & 5.465 & 33.448 & 8.095 \\
Pollution & 2.090 & 14.217 & 0.819 & 15.073 \\
CBD Distance & 11.757 & 9.342 & 12.565 & 8.936 \\
Poverty & 0.107 & 0.102 & 0.122 & 0.121 \\
Grad Degree & 0.092 & 0.087 & 0.081 & 0.085 \\
Black & 0.156 & 0.239 & 0.237 & 0.342 \\
Gay/Lesbian & 0.007 & 0.006 & 0.005 & 0.004 \\
Income & 2.271 & 0.916 & 2.193 & 1.140 \\
Age Sq & 0.262 & 0.312 & 0.390 & 0.333 \\
House Size Sq & 2.922 & 1.509 & 3.017 & 2.337 \\
Lot Size Sq & 13.836 & 46.877 & 22.400 & 145.016 \\
Observations & & & & \\
\hline
\end{tabular}


Table 4.3: Spatial Autoregressive Hedonic Price Model Results for Cleveland and Columbus MSAs (Dependent Variable is the Natural Log of House Price)

\begin{tabular}{|c|c|c|c|c|c|c|}
\hline Variable & $\begin{array}{l}\text { Columbus } \\
\quad(1) \\
\text { Coefficient }\end{array}$ & T-stat & $\begin{array}{l}\text { Cleveland } \\
\quad(2) \\
\text { Coefficient }\end{array}$ & T-stat & $\begin{array}{l}\text { Cleveland } \\
\text { (3) } \\
\text { Coefficient }\end{array}$ & T-stat \\
\hline CBD Distance & -0.0037 & $-2.23^{* *}$ & -0.0027 & $-3.09 * * *$ & -0.0051 & $-4.55 * * *$ \\
\hline Age & -0.4554 & $-3.56^{* * *}$ & 0.1599 & 1.30 & 0.1746 & 1.56 \\
\hline Age $\mathrm{Sq}$ & 0.3281 & $2.99 * * *$ & -0.4390 & $-4.72^{* * *}$ & -0.4001 & $-4.71^{* * *}$ \\
\hline House Size & 0.7823 & $4.81^{* * *}$ & 0.4321 & $8.03^{* * *}$ & 0.3641 & $6.42^{* * *}$ \\
\hline House Size Sq & -0.1110 & $-2.87 * * *$ & -0.0107 & -1.25 & -0.0047 & -0.44 \\
\hline Lot Size & 0.0256 & $3.68^{* * *}$ & 0.0059 & $2.14^{* *}$ & 0.0104 & $3.63^{* * *}$ \\
\hline Lot Size Sq & -0.0009 & $-2.35^{* *}$ & 0.0000 & -0.32 & -0.0001 & -1.43 \\
\hline Partial Bath & 0.1652 & $2.85^{* * *}$ & 0.1012 & $3.21^{* * *}$ & 0.1028 & $3.32^{* * *}$ \\
\hline Full Bath & 0.1159 & $2.27^{* *}$ & 0.0137 & 0.37 & 0.0284 & 0.84 \\
\hline Deck & 0.1736 & $1.92^{*}$ & 0.1485 & $2.97^{* * *}$ & 0.2232 & $4.48 * * *$ \\
\hline Garage & -0.0412 & -0.98 & 0.0190 & 0.66 & 0.1733 & $4.45^{* * *}$ \\
\hline One Story & 0.1365 & $3.92^{* * *}$ & 0.0435 & 1.56 & -0.0016 & -0.05 \\
\hline Air Conditioner & 0.1095 & $2.22^{* *}$ & -0.0931 & $-3.09 * * *$ & -0.0125 & -0.31 \\
\hline Income & 0.0736 & $3.49 * * *$ & 0.0414 & $4.55^{* * *}$ & 0.0423 & $4.63^{* * *}$ \\
\hline Grad Degree & 1.0870 & $6.85^{* * *}$ & 0.5753 & $4.40^{* * *}$ & 0.5756 & $4.43^{* * *}$ \\
\hline School Quality & 0.0009 & 1.26 & 0.0026 & $6.11^{* * *}$ & 0.0026 & $6.51^{* * *}$ \\
\hline Tax Rate & 0.0018 & 0.90 & -0.0018 & $-2.37 * *$ & -0.0020 & $-2.51^{* *}$ \\
\hline Poverty & -0.0373 & -0.30 & -0.2156 & $-2.14^{* *}$ & -0.1578 & $-1.81^{*}$ \\
\hline Pollution & 0.0004 & 1.12 & 0.0006 & $1.90^{*}$ & 0.0006 & $1.81^{*}$ \\
\hline Black & -0.1619 & $-4.51^{* * *}$ & -0.1096 & $-5.24 * * *$ & -0.0978 & $-4.67 * * *$ \\
\hline Gay/Lesbian & 8.0524 & $4.61^{* * *}$ & 4.3508 & $2.31^{* *}$ & 4.0251 & $2.32^{* *}$ \\
\hline Gay/Lesbian*Conservative & -10.4204 & $-2.00 * *$ & 0.2967 & 0.07 & & \\
\hline Conservative & 0.1777 & $2.83^{* * *}$ & -0.0074 & -0.30 & & \\
\hline Gay/Lesbian*Geauaga & & & & & -31.5364 & $-1.68^{*}$ \\
\hline Gay/Lesbian*Lake & & & & & -0.0752 & -0.02 \\
\hline Gay/Lesbian*Lorain & & & & & 3.8992 & 0.56 \\
\hline Gay/Lesbian*Medina & & & & & -9.4998 & -0.74 \\
\hline Geauaga & & & & & 0.1283 & $1.79^{*}$ \\
\hline Lake & & & & & 0.2497 & $5.06 * * *$ \\
\hline Lorain & & & & & 0.1408 & $3.46^{* * *}$ \\
\hline Medina & & & & & 0.0801 & 1.59 \\
\hline rho & 0.1072 & & 0.1569 & & 0.1576 & \\
\hline r squared & 0.92 & & 0.93 & & 0.94 & \\
\hline observations & 367 & & 653 & & 653 & \\
\hline
\end{tabular}

a Statistical significance is reported at the $1 \%(* * *), 5 \%(* *)$, and $10 \%(*)$ levels.

b The Gay/Lesbian, Black, Poverty, and Grad Degree neighborhood characteristics enter the regression as proportions. Therefore, the coefficients reported in the table for these variables indicate the percent change in house prices resulting from a one percentage point increase in the variable. 


\section{Chapter 5}

\section{Conclusion}

The three essays of this dissertation provide empirical evidence that informs the debate over the passage of gay and lesbian rights legislation. Chapter 2 provides evidence that the passage of a sexual orientation private employment anti-discrimination law does not have the effect that it is intended to have by its passage. The passage of this law has no impact on the employment outcomes for lesbians, while it has a mixed impact on these outcomes

for gays. Specifically, gays realize hire relative earnings but less of a relative probability of finding employment as a result of the passage of this law. The higher earnings are received by younger gays and the lower chance of finding employment is a result only experienced by older gays. These findings indicate that resources used by gay and lesbian rights groups, as well as politicians, in attempting to get a sexual orientation anti-discrimination law passed can likely be put to use in other ways that have more of a positive impact on the lives of gays and lesbians.

Chapter 2 only focuses on the passage of eight private employment anti-discrimination laws due to only having the 1990 and 2000 Census data to work with. Since 1998, ten more states have passed the private employment anti-discrimination law. With the release of more recent data on gays and lesbians the analysis performed in Chapter 2 can be extended to look at the impact of the passage of this recent legislation, and how the impact of this law 
has changed overtime. Also for future research, the development of a theory is necessary towards why the labor market outcomes of different groups of individuals, such as blacks, women, the disabled, gays, lesbians, and different gay age groups, are impacted differently by anti-discrimination legislation.

Chapter 3 indicates that gays and lesbians do have preferences for particular types of gay and lesbian rights legislation. It is found that these individuals prefer to live in states with hate crime and public employment anti-discrimination laws, and that they may prefer to not live in states with private employment anti-discrimination laws. Therefore, the evidence from chapter 3 suggests that the recently passed federal sexual orientation hate crime law has increased the utility of gays and lesbians. What it also suggests is that the passage of the federal private employment anti-discrimination law that is currently a proposed bill in Congress could possibly reduce the utility of gays and lesbians. Additionally, these revealed preference findings show that gays and lesbians are likely to benefit from the passage of a public employment anti-discrimination law at the state or federal level of government.

As for future research, recent data on gay and lesbian migration can be used to analyze how the passage of the legislation analyzed in chapter 3 , as well as the passage of recently passed same sex marriage and union laws, have hurt or benefited gays and lesbians. Extending the analysis to these recently passed relationship laws would be particularly important for an analysis of how state and local areas can attract gays and lesbians into their regions. This is because only a few states have passed these laws, so large gains of gays and lesbians could possibly be had with the passage of this relationship legislation. Also, it appears that a federal law allowing for gays and lesbians to enter into unions or marriage is a long way off. Therefore, the passage of this legislation at the state and local level is likely to have a long term impact on the gay and lesbian population of the region. Lastly, as with any migration model of public policies, endogeneity of gay and lesbian rights legislation is a possibility in the model presented in chapter 3. With more gay and lesbian migration data over time, difference-in-difference approaches using panel data can be used to analyze the possibility 
and impact of this endogenity in the context of gay and lesbian rights legislation.

Chapter 4 provides results that are consistent with the theory that prejudice exists against gay and lesbian households in conservative neighborhoods, and that these households are actually an amenity to liberal neighborhoods. This finding suggests that arguments and votes made against the passage of gay and lesbian rights legislation may be the result of prejudice that is held by individuals, rather than an honest analysis of the costs and benefits of the legislation. This finding is important because the rejection of any type of legislation should not be the result of hidden prejudice on the part of voters or politicians. The policy implications of this finding are unclear because it is hard, if not impossible, to change prejudice that is held by individuals in society and in policy making roles in the government. It is necessary though to consider that prejudice may be behind negative claims being made regarding the passage of gay and lesbian rights legislation in order to fully understand these claims.

Future research extensions for chapter 4 are numerous. For one, it is necessary to come up with a more precise and localized measure of the social conservativeness of a neighborhood. The George Bush vote proxy used in chapter 4 is the best one available based on the sample data, but using a county measure such as this is far from perfect. Additionally, it should be kept in mind that this analysis is based on data from two MSAs in the same state, and on a single data set. It would be beneficial to run a similar analysis as in chapter 4 using a different data set of housing prices covering different areas of the country. Also, the creation of a data set on housing purchases that includes the sexual orientation of the households involved in the transactions is of great importance, as this would allow an analysis of if prejudice results in housing market discrimination against gays and lesbians. 


\section{Bibliography}

Acemoglu, Daron and Joshua D. Angrist, "Consequences of Employment Protection? The Case of the Americans with Disabilities Act," The Journal of Political Economy, 2001, $109(5), 915-957$.

Allegretto, Sylvia A. and Michelle M. Arthur, "An Empirical Analysis of Homosexual/Heterosexul Male Earnings Differentials: Unmarried and Unequal?," Industrial and Labor Relations Review, 2001, 54 (3), 631-646.

Antecol, Heather, Anneke Jong, and Michael Steinberger, "The Sexual Orientation Wage Gap: The Role of Occupational Sorting and Human Capital," Industrial and Labor Relations Review, 2008, 61 (4), 518-543.

Ashby, Nathan, "Economic Freedom and Migration Flows Between U.S. States," Southern Economic Journal, 2007, 73 (3), 677-697.

Association of Religious Data Archives, Churches and Church Membership in the United States, 1990 (States) 1990. http://www.thearda.com/Archive/Files/ Descriptions/CMS90ST.asp (Accessed April 08, 2010).

Badgett, M. V. Lee, "The Wage Effects of Sexual Orientation Discrimination," Industrial and Labor Relations Review, 1995, 48 (4), 726-739.

_, Money, Myths, and Change: The Economic Lives of Lesbians and Gay Men, Chicago: The University of Chicago Press, 2001.

Bailey, Martin J., "Effects of Race and Other Demographic Factors on the Values of Single-Family Homes," Land Economics, 1966, 42 (2), 215-220.

Becker, Gary S., The Economics of Discrimination, 2nd ed., Chicago: University of Chicago Press, 1971.

Beegle, Kathleen and Wendy A. Stock, "The Labor Market Effects of Disability Discrimination Laws," The Journal of Human Resources, 2003, 38 (4), 806-859.

Bell, David and Jon Binnie, "Authenticating Queer Space: Citizenship, Urbanism and Governance," Urban Studies, 2004, 41 (9), 1807-1820.

Berg, Nathan and Donald Lien, "Measuring the Effect of Sexual Orientation on Income: Evidence of Discrimination?," Contemporary Economic Policy, 2002, 20 (4), 394-414. 
Bertrand, Marianne, Esther Duflo, and Sendhil Mullainathan, "How Much Should We Trust Differences-in-Differences Estimates?," 2002. NBER Working Paper 8841.

Besley, Timothy and Anne Case, "Unnatural Experiments? Estimating the Incidence of Endogenous Policies," The Economic Journal, November 2000, 110, F672-F694.

Black, Dan A., Hoda R. Makar, Seth G. Sanders, and Lowell J. Taylor, "The Earnings Effects of Sexual Orientation," Industrial and Labor Relations Review, 2003, 56 (3), 446-469.

_, Seth G. Sanders, and Lowell J. Taylor, "Demographics of the Gay and Lesbian Population in the United States: Evidence from Available Systematic Data Sources," Demography, 2000, 37 (2), 139-154.

Black, Dan, Gary Gates, Seth Sanders, and Lowell Taylor, "Why Do Gay Men Live in San Francisco?," Journal of Urban Economics, 2002, 51 (1), 54-76.

Blanford, John M., "The Nexus of Sexual Orientation and Gender in the Determination of Earnings," Industrial and Labor Relations Review, 2003, 56 (4), 622-642.

Blank, Rebecca, "The Effect of Welfare and Wage Levels on the Location Decisions of Female Headed Households," Journal of Urban Economics, 1988, 24 (2), 186-211.

Borjas, George, "Economic Theory and Internal Migration," International Migration Review, 1989, 23 (3), 457-485.

Brasington, David M., "Private Schools and the Willingness to Pay for Public Schooling," Education Finance and Policy, 2007, 2 (2), 152-174.

- and Diane Hite, "Demand for Environmental Quality: A Spatial Hedonic Analysis," Regional Science and Urban Economics, 2005, 35, 57-82.

_ and _ , "A Mixed Index Approach to Identifying Hedonic Price Models," Louisiana State Univerisity Department of Economics 2006.

- and Donald R. Haurin, "Educational Outcomes and House Values: A Test of the Value-Added Approach," Journal of Regional Science, 2006, 46 (2), 245-268.

Carpenter, Chris, "New Evidence on Gay and Lesbian Household Incomes," Contemporary Economic Policy, 2004, 22 (1), 78-94.

_ , "Self-Reported Sexual Orientation and Earnings: Evidence from California," Industrial and Labor Relations Review, 2005, 58 (2), 258-272.

Cebula, Richard and James Koch, "Welfare Policies and the Migration of the Poor in the United States," Public Choice, 1989, 61, 171-176.

Clain, Suzanne Heller and Karen Leppel, "An Investigation Into Sexual Orientation Discrimination as an Explanation for Wage Differences," Applied Economics, 2001, 33 (1), 37-47. 
Clark, David E. and William J. Hunter, "The Impact of Economic Opportunity, Amenities, and Fiscal Factors on Age-Specific Migration Rates," Journal of Regional Science, 1992, 32 (3), 349-365.

Collins, Williams, "The Labor Market Impact of State-Level Anti-Discrimination Laws, 1940-1960," Industrial and Labor Relations Review, 2003, 56 (2), 244-272.

Conway, Karen and Andrew J. Houtenville, "Elderly Migration and State Fiscal Policy: Evidence from the 1990 Census Migration Flow," National Tax Journal, 2001, 54 (1), $103-124$.

_ and _, "Out with the Old, In with the Old: A Closer Look at Younger Versus Older Elderly Migration," Social Science Quarterly, 2003, 84 (2), 309-328.

- and Jonathan C. Rork, "State "Death" Taxes and Elderly Migration-The Chicken or the Egg?," National Tax Journal, 2006, 59 (1), 97-128.

Cushing, Brian and Jacques Poot, "Crossing Boundaries and Borders: Regional Science Advances in Migration Modeling," Papers in Regional Science, 2004, 83, 317-338.

Cushing, Brian J., "A Note on Specification of Climate Variables in Models of Population Migration," Journal of Regional Science, 1987, 27 (4), 641-649.

Egan, Patrick J., Murray S. Edelman, and Kenneth Sherrill, "Findings from the Hunter College Poll of Lesbians, Gays, and Bisexuals: New Discoveries about Identity, Political Attitudes, and Civic Engagement," Technical Report, Hunter College 2008.

Elmslie, Bruce and Edinalo Tebaldi, "Sexual Orientation and Labor Market Discrimination," Journal of Labor Research, 2007, 28, 436-453.

Fields, Gary S., "Place-to-Place Migration: Some New Evidence," The Review of Economics and Statistics, 1979, 61 (1), 21-32.

Florida, Richard, "Bohemia and Economic Geography," Journal of Economic Geography, 2002, $2(1), 55-71$.

Fournier, Gary M., David W. Rasmussen, and William J. Serow, "Elderly Migration as a Response to Economic Incentives," Social Science Quarterly, 1988, 69 (2), 245-260.

Gates, Gary J., "Geographic Trends Among Same-Sex Couples in the U.S. Census and the American Community Survey," Williams Institute, UCLA School of Law 2007.

Graves, Phillip, "A Life Cycle Empirical Analysis of Migration and Climate," Journal of Urban Economics, 1979, 6 (2), 135-147.

Greenwood, Michael J., "Research on Internal Migration in the United States: A Survey," Journal of Economic Literature, 1975, 13 (2), 397-433.

- and Gary L. Hunt, "Jobs Versus Amenities in the Analysis of Metropolitan Migration," Journal of Urban Economics, 1989, 25 (1), 1-16. 
_, _, and John M. McDowell, "Migration and Employment Change: Empirical Evidence on the Spatial and Temporal Differences of the Linkage," Journal of Regional Science, 1986, 26 (2), 223-234.

Haider-Markel, Donald P. and Kenneth J. Meier, "The Politics of Gay and Lesbian Rights: Expanding the Scope of the Conflict," The Journal of Politics, 1996, 58 (2), 332-349.

Harris, David R., "Property Values Drop When Blacks Move In Because: Racial and Socioeconomic Determinants of Neighborhood Desirability," American Sociological Review, 1999, 64 (3), 461-479.

Heckman, James, "Sample Selection Bias as a Specification Error," Econometrica, 1979, 47, 153-161.

- , "The Central Role of the South in Accounting for the Economic Progress of Black Americans," American Economic Review, 1990, 80 (2), 242-260.

Herek, Gregory M., "Hate Crimes and Stigma-related Experiences Among Sexual Minority Adults In the United States: Prevalence Estimates From a National Probability Sample," Journal of Interpersonal Violence, 2009, 24 (1), 54-74.

Human Rights Campaign, State Laws and Policies 2009. http://www.hrc.org/ documents/Employment_Laws_and_Policies.pdf(Accessed October 31, 2009).

Kennedy, Peter M., A Guide to Econometrics (6E), Malden, MA: Blackwell Publishing, 2008.

Kiel, Katherine A. and Jeffrey E. Zabel, "House Price Differentials in U.S. Cities: Household and Neighborhood Racial Effects," Journal of Housing Economics, 1996, 5 (2), 143-165.

Klawitter, Marieka and Victor Flatt, "The Effects of State and Local Antidiscrimination Policies on Earnings for Gays and Lesbians," Journal of Policy Analysis and Management, 1998, 17 (4), 658-686.

Langbein, Laura and Mark A. Yost Jr., "Same-Sex Marriage and Negative Externalities," Social Science Quarterly, 2009, 90 (2), 292-308.

Lee, Ming and R. Kelley Pace, "Spatial Distribution of Retail Sales," Journal of Real Estate Finance and Economics, 2005, 31 (1), 53-69.

Leguizamon, Susane, "The Influence of Reference Group House Size on House Price," Real Estate Economics, 2010. Forthcoming.

LeSage, James and R. Kelley Pace, "Spatial Econometric Modelling of OriginDestination Flows," Journal of Regional Science, 2008, 48 (5), 941-947.

_ and _, Introduction to Spatial Econometrics, Boca Raton: Chapman and Hill, 2009. 
Library of Congress, THOMAS 2009. http://thomas.loc.gov/cgi-bin/bdquery/z? d111:S1584:(Accessed October 31, 2009).

-, THOMAS 2010. http://thomas.loc.gov/cgi-bin/bdquery/z?d111: SP1511:(Accessed April 7, 2010).

Myers, Caitlin Knowles, "Discrimination and Neighborhood Effects: Understanding Racial Differences in US Housing Prices," Journal of Urban Economics, 2004, 56 (2), 279-302.

National Gay and Lesbian Task Force, Capital Gains and Losses: A State by State Review of Gay-Related Legislation in 19981998.

National Opinion Research Center at the University of Chicago, General Social Survey. http://www.norc.org/GSS+Website/ (Accessed April 08, 2010).

Neumark, David and Wendy Stock, "The Effects of Race and Sex Discrimination Laws," 2001. NBER Working Paper 8215.

Palmquist, Raymond B., "Estimating the Demand for the Characteristics of Housing," Review of Economics and Statistics, 1984, 66 (3), 394-404.

Rimmerman, Craig A., Kenneth D. Wald, and Clyde Wilcox, eds, The Politics of Gay Rights, Chicago: University of Chicago Press, 2000.

Roback, Jennifer, "Wages, Rents, and the Quality of Life," The Journal of Political Economy, 1982, 90 (6), 1257-1278.

Rosen, Sherwin, "Hedonic Prices and Implicit Markets: Product Differentiation in Pure Competition," The Journal of Political Economy, 1974, 82 (1), 34-55.

Rubenstein, William B., "Do Gay Rigts Laws Matter?: An Empirical Assessment," Southern California Law Review, 2001, 75, 65-120.

Ruggles, Steven, Matthew Sobek, Trent Alexander, Catherine A. Fitch, Ronald Goeken, Patricia Kelly Hall, Miriam King, and Chad Ronnander, "Integrated Public Use Microdata Series: Version 4.0 [Machine-readable database]," Minneapolis: Minnesota Population Center [producer and distributor] 2009.

Tebaldi, Edinaldo and Bruce Elmslie, "Sexual Orientation and Labour Supply," Applied Economics, 2006, 38, 549-562.

Tiebout, Charles M., "A Pure Theory of Local Expenditures," Journal of Political Economy, 1956, 64 (5), 416-424.

Tiefelsdorf, Michael, "Misspecifications in Interaction Model Distance Decay Relations: A Spatial Structure Effect," Journal of Geographical Systems, 2003, 5 (1), 25-50. 
U.S. Census Bureau, Population Division, Technical Note on Same-Sex Unmarried Partner Data From the 1990 and 2000 Censuses 2008. http://www.census.gov/ population/www/cen2000/samesex/index.html(Accessed September 10, 2008).

Yinger, John, "Racial Prejudice and Racial Residential Segregation in an Urban Model," Journal of Urban Economics, 1976, 3 (4), 383-396. 